\title{
Metallurgy Division Fy 2003 Programs and Accomplishments
}

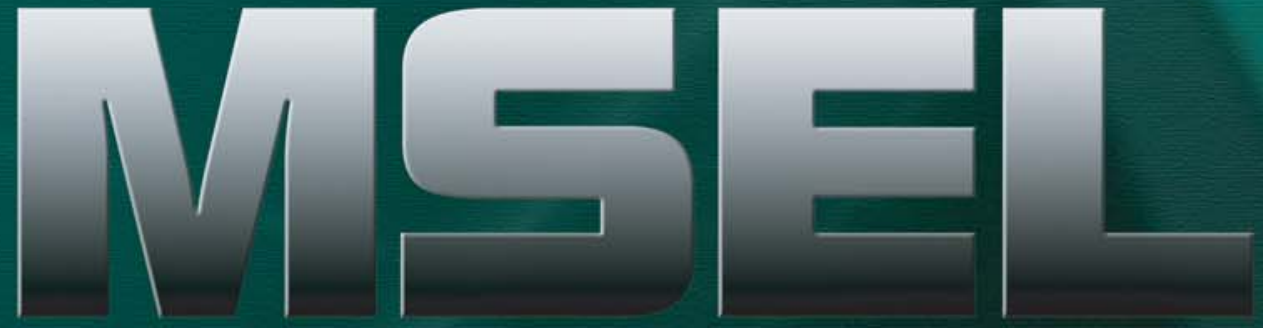

\section{NGT}

National Institute of

Standards and Technology

Technology Administration

U.S. Department of

Commerce

NISTIR 7017

September 2003 


\section{On the Cover:}

From the front cover and continuing on to the back, the images shown are, respectively:

Fig 1 - Phase field simulation of dendritic growth in polymers.

Fig 2 - Simulated polycrystalline microstructure developed for modeling magnetic damping.

Fig 3 - Contributions of grains oriented in specific ways to line broadening in ferromagnetic resonance measurements.

Fig 4 - Composition map of $\mathrm{Ni} / \mathrm{Cu}$ electrodeposited multilayer nanowires.

Fig 5 - Optical micrograph of the deformation of alloy 7075 resulting from high-speed machining operations. 
National Institute of

Standards and Technology

Arden L. Bement, Jr.

Director

Technology

Administration

Phillip J. Bond

Undersecretary of

Commerce for Technology

U.S. Department

of Commerce

Donald L. Evans

Secretary

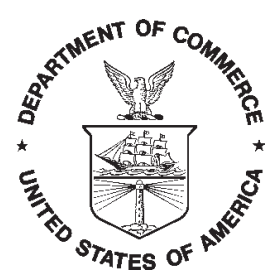

\section{Materials Science and Engineering Laboratory}

\section{FY 2003 Programs and ACcOMplisHMENTS}

\section{Metallurgy \\ Division}

Carol A. Handwerker, Chief

Frank W. Gayle, Deputy

NISTIR 7017

September 2003 



\section{Table of Contents}

Executive Summary 1

Technical Highlights 3

Solidification in the Presence of Impurities:

Phase Field Simulations of the Growth of "Dizzy” Dendrites .......................................... 4

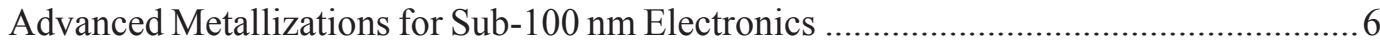

High Throughput Data Analysis of Multicomponent Multiphase Diffusion Data

Modeling Dynamics, Damping and Defects for Perpendicular

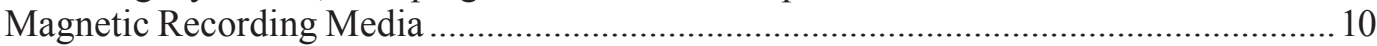

Artifacts in Ballistic Magnetoresistance Measurements ................................................. 12

Phase Field Modeling of the Electrochemical Interface .................................................. 14

NEMI-NIST-TMS Workshop on Tin Whiskers: Cause and Effect ............................... 16

Kolsky Bar: Materials Properties at High Strain Rates and

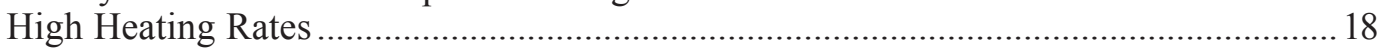

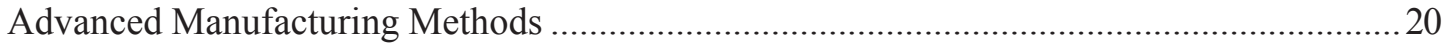

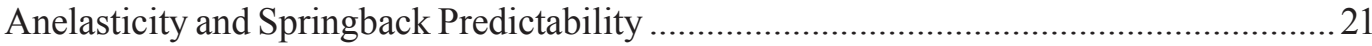

Microstructural Origins of Surface Roughening and

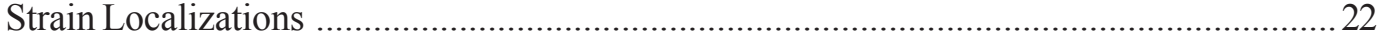

Plasticity, Fabrication Processes, and Performance ……................................................. 23

Standard Tests and Data for Sheet Metal Formability ……….........................................2

Underlying Processes of Plastic Deformation in Metal Alloys ........................................25

Atomization Processing — Recommended Practices .................................................... 26

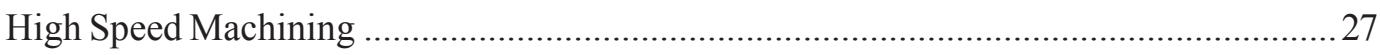

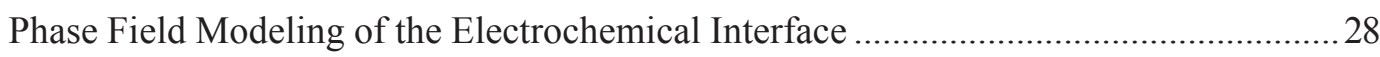

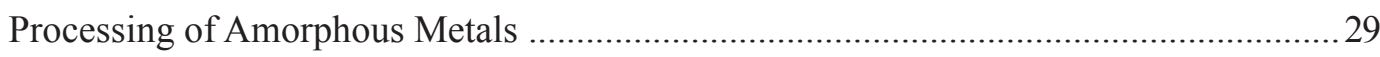

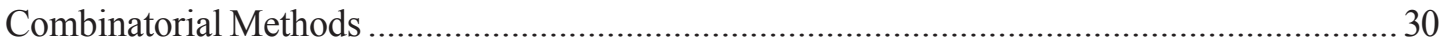

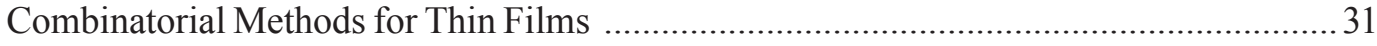

Phase Diagrams for III-V Thin Films and Metal Contacts .............................................32

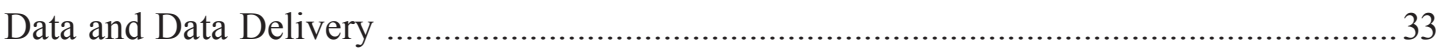

Informatics and Visualization in Materials Data Delivery ............................................... 34

Reaction Path Analysis in Multicomponent Systems …....................................................35 


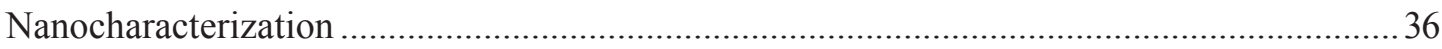

Bridging Length Scales in Theory and Modeling:

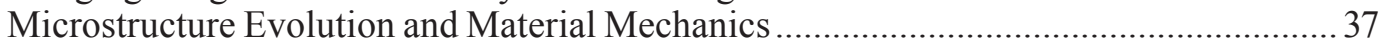

Electrochemical Processing of Nanoscale Materials .......................................................... 38

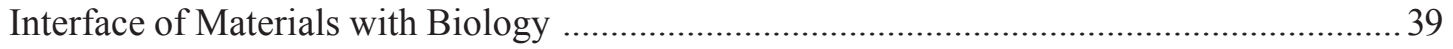

Biocompatibility of Metals Reference Powders ...............................................................40

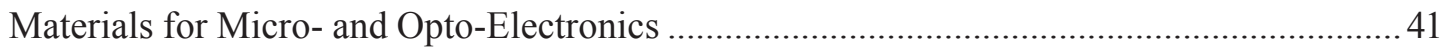

Superconformal Film Growth: Measurement and Modeling ......................................... 42

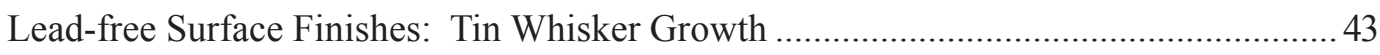

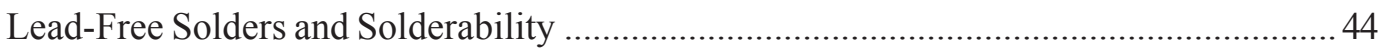

Magnetic Properties and Standard Reference Materials ................................................ 45

Magnetic Properties of Nanostructured Materials .......................................................... 46

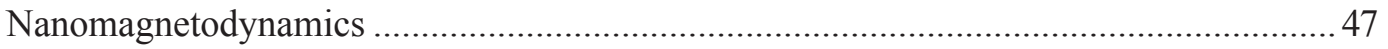

Anomalously Large Low Temperature Intermixing in

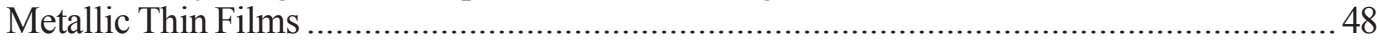

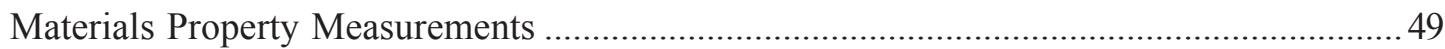

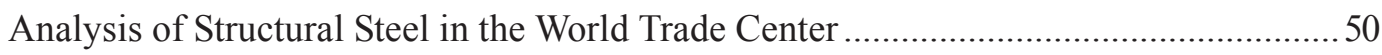

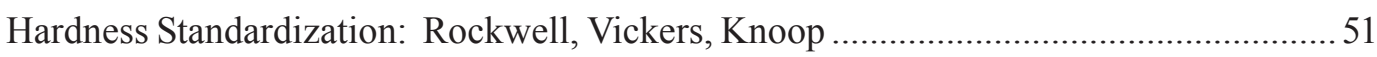

Marine Forensics and Preservation of Historic Shipwrecks ...........................................52

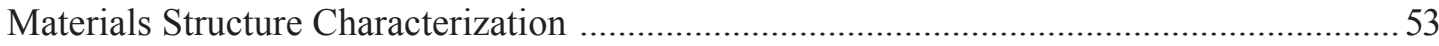

Nanoscale Characterization by Electron Microscopy …....................................................5

USAXS Imaging: Microscope Development and Validation ...........................................55

Metallurgy Division FY03 Annual Report Publication List ............................................. 57

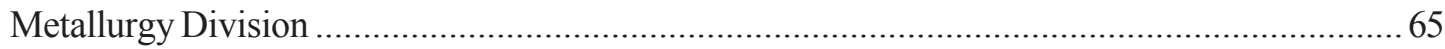

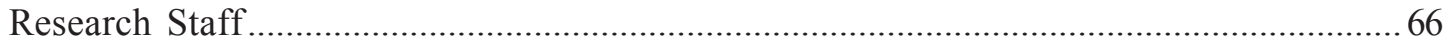

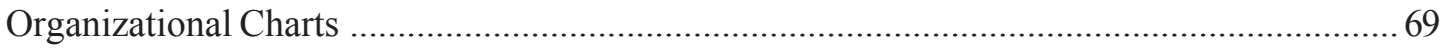




\section{Executive Summary}

This Annual Report describes the major technical activities, accomplishments, and areas of scientific expertise in the Metallurgy Division of NIST Materials Science and Engineering Laboratory for FY2003 (October 2002 through September 2003). Through this report, we provide some insight into how our research programs meet the needs of our customers, how the capabilities of the Metallurgy Division are being used to solve problems important to the national economy and the materials metrology infrastructure, and how we interact with our customers to establish new priorities and programs. Contact information for our staff is provided if you would like to participate in any of these projects or if have any questions. We also welcome feedback and suggestions on how we can better serve the needs of our customers and encourage increasing collaboration to this end.

\section{Mission of the NIST Metallurgy Division}

Our mission is to provide critical leadership in the development of measurement methods, standards, and fundamental understanding of materials behavior needed by U.S. materials users and producers to become or remain competitive in the changing global marketplace.

As a fundamental part of this mission, we are responsible not only for developing new measurement methods with broad applicability across materials classes and industries but also for working with individual industry groups to develop and integrate measurements, standards, software tools, and evaluated data for specific, technologically important applications.

\section{Establishing Priorities}

We examine a wide range of research opportunities and make choices for our research portfolio based on: the match to the NIST mission, the magnitude and immediacy of industrial need, whether the NIST contribution is critical for success, the anticipated impact relative to our investment, our ability to respond in a timely fashion with high-quality output, and the opportunity to advance mission science. This requires us to establish our research priorities through extensive consultation and collaboration with our customers in U.S. industry and with our counterparts in the international metrology community using a variety of methods including industrial roadmapping activities, workshops, technical meetings, standards committee participation, and individual consultation with our customers. For us to have the highest possible impact also requires that we work to be at the forefront of materials science.
Within the context of industrial relevance and potential impact of our research, technology trends strongly influence the technical directions addressed by NIST. We prefer to work in rapidly evolving technologies, where advances in measurement science are needed to understand the limitations on system behavior and, therefore, where our contributions are likely to have an impact on the course of technology. For NIST as a whole and the Metallurgy Division in particular, we are committed to having an impact on the measurement and standards infrastructure for nanotechnology, health care, and homeland security. Nanotechnology is not an industry sector itself but will have an impact on present and future industrial enterprises. Three new Division projects began in nanotechnology and health care in 2003; an additional project on Informatics and Visualization in Data Delivery was started in 2003 to examine how to best transfer our results to traditional and possible NIST customers. In 2004, we will begin two new nanotechnology projects selected through a NIST-wide competition. In collaboration with three other NIST laboratories (EEEL, ITL, PL), a major project will be started on Nanoscale Engineered Sensors for Ultra-low Magnetic Field Metrology. Low noise, ultra-low magnetic field sensors could have a significant impact on healthcare diagnostics, homeland security sensors, and magnetic data storage. In collaboration with three other NIST laboratories (CSTL, PL, and ITL), we will begin developing systems for three-dimensional chemical imaging at the nanoscale in the transmission electron microscope. This should add greatly to our understanding of the relationship between materials composition, processing, and properties, the raison d'etre of materials science and engineering. These were the only two fully funded projects in the NIST competition.

\section{Research Portfolio}

Our 2003 research portfolio focuses on fulfilling specific measurement needs of the industrial sectors: the magnetic data storage, microelectronics/ optoelectronics, automotive, aerospace, and defense industries, and on developing the materials data need for the World Trade Center failure investigation. Our output consists of a variety of forms, from a fundamental understanding of materials behavior to measurement techniques conveyed through the scientific literature and oral presentations, standard reference materials, evaluated data and online databases, software tools, and sensors for on-line process control. Examples of our 2003 outputs in a few of these areas are:

- Magnetic Data Storage: In 2003, we shifted our emphasis from Giant Magnetoresistance (GMR) in Thin Films to understanding artifacts in Ballistic Magnetoresistance (BMR). In the past year, advances 
in the Ballistic Magnetoresistance (BMR) effect appeared to provide an enormous increase in the sensitivity of nanoscale devices. The scientific press reported the creation of devices with resistance changes as much as $10^{6}$ as one of the major discoveries of the year. Experiments at NIST in 2003 have found that several simple artifacts account for the results. NIST is taking a leading role in designing artifact-free metrology to provide reliable data to the magnetic sensor industry and the academic community.

\section{- Microelectronics Packaging: In the MSEL} Program on Materials for Microelectronics, we continue to provide tools for producing improved metal interconnects, from copper on-chip interconnects at the nanometer scale to lead-free solder joints on printed wiring boards. Our project on measurements and modeling of electrodeposited copper for nanometer scale chip interconnection technology continues to produce significant value to the microelectronics community. Since the beginning of the project in April 1999, we have developed a measurement technique, a theory for control of interface dynamics, and modeling software for predicting quantitatively the ability of complex electrolytes to fill vias and trenches, and we extended the NIST model to electrodeposited metals other than copper. In 2003, we used our theory as a basis for developing processing methods to increase the aspect ratios of fine features $(<50 \mathrm{~nm})$ that can be filled and have transferred all of these to the appropriate industrial customers. In addition, in response to the need for improved seed layer systems articulated to us by International Sematech, we demonstrated the utility of thin ruthenium layers as combination barrier layer/seed layer in superconformal growth on patterned substrates. In the area of electronics assembly, our role in the NEMI Lead-Free Solder Task Force has wound down. With the completion of Task Force research plan in 2002, and final analysis of the results in 2003, U.S. microelectronics companies now have enough critically evaluated data to decide on whether to implement lead-free solders in manufacturing. The one open question is how important tin whiskers forming on plated component surface finishes will be for system reliability. There is a long, documented history of tin whiskers causing catastrophic short circuits in critical systems such as satellites and medical devices. In the past, $\mathrm{Pb}$-Sn alloys were plated instead of $\mathrm{Sn}$ since $\mathrm{Pb}$ additions appeared to suppress whisker growth. With the requirement of $\mathrm{Pb}$-free manufacturing in some regions, such as the European union, $\mathrm{Pb}$ additions are no longer acceptable. NIST has taken a major role in helping NEMI develop test methods for whisker growth and a fundamental understanding of the mechanisms leading to whisker formation.

- Automotive: Within the sub-program on the Forming of Lightweight Materials, we are developing standard test methods for sheet metal forming, measurements of surface roughness, and physically based constitutive laws - and measurement tools needed to reveal them. In FY2003, an X-ray stress measuring system was installed on the sheet metal formability station. With this addition, the formability facility will be capable of direct, in situ measurement of the stress in a given direction while the sample is under biaxial load. Such measurements will lead to a standard test for measuring multiaxial flow surfaces in sheet metal during stamping operations. All of our projects in the Forming of Lightweight Materials Program are performed in close collaboration with the automotive industry through formal partnerships, such as USCAR and the Freedom Car, and are formulated to help accelerate the design of forming operations for lightweight materials such as aluminum, that will ultimately improve fuel economy.

- Infrastructure Materials: NIST has developed a unique testing facility for measuring high-strain rate mechanical properties of materials at high heating rates. Based on the Kolsky bar design (also known as a split-Hopkinson pressure bar), materials can be strained at rates as high as $10^{5} \mathrm{~s}^{-1}$ while being heated up to $1300 \mathrm{~K}$ at heating rates up to $5 \times 10^{4} \mathrm{~K} / \mathrm{s}$. This facility was initially developed to provide insight and data for high speed machining of commercial alloys. In addition to this application, the Kolsky bar facility is providing the rate dependence of plastic deformation for the many types of structural steel in the World Trade Center buildings and for projectiles such as those used as military tank penetrators. We expect the facility to be useful in the future for development of data and modeling in the areas of dynamic fracture and crack arrest of high strength pipeline steels, bullets and body armor for the Office of Law Enforcement Standards, and additional issues in manufacturing.

\section{Division Structure and Expertise}

The Division is composed of 39 scientists, supported by 6 technicians, 6 administrative staff members, and more than 80 guest scientists, and it's organized into five groups that represent the Division's core expertise in Metallurgical Processing, Electrochemical Processing, Magnetic Materials, Materials Structure and Characterization, and Materials Performance. However, by virtue of the interdisciplinary nature of materials problems in the industrial and metrology sectors that we serve, Program teams are assembled across group, division and laboratory boundaries and, in most cases, with academic and industrial partners, to best meet our project goals. We are committed to assembling the expertise and resources to fulfill our technical goals with the speed and quality necessary to have the desired impact. We welcome your participation with us in this enterprise.

Carol A. Handwerker, Division Chief

Frank W. Gayle, Deputy Division Chief 


\section{Technical Highlights}

The following Technical Highlights section includes expanded descriptions of research projects that have broad applicability and impact. These projects generally continue for several years. The results are the product of the efforts of several individuals. The Technical Highlights include:

- Solidification in the Presence of Impurities: Phase Field Simulations of the Growth of "Dizzy" Dendrites

- Advanced Metallizations for Sub-100 nm Electronics

- High Throughput Data Analysis of Multicomponent Multiphase Diffusion Data

- Modeling Dynamics, Damping and Defects for Perpendicular Magnetic Recording Media

- Artifacts in Ballistic Magnetoresistance Measurements

- Phase Field Modeling of the Electrochemical Interface

- NEMI-NIST-TMS Workshop on Tin Whiskers: Cause and Effect

- Kolsky Bar: Materials Properties at High Strain Rates and High Heating Rates 


\section{Solidification in the Presence of Impurities: Phase Field Simulations of the Growth of "Dizzy" Dendrites}

It is beyond the capacity of conventional models of solidification to describe and predict the formation and growth of polycrystalline structures. With the NIST development of a new phase field model of polycrystals, entire classes of microstructure are now subject to study not only within the confines of the laboratory but also the theoretician's computer. This computational tool offers unprecedented control in the design of crystalline and semi-crystalline material.

\section{James A. Warren and Jack Douglas}

$\mathrm{D}$ uring solidification, materials do not freeze in a uniform manner, except under very special circumstances. Instead, a whole host of patterns emerges, of which the snowflake is the most familiar. The snowflake is not unique to water, however, as nearly all materials can solidify having such a pattern, and this process is referred to as dendritic growth. Dendrites, Latin for "trees," are ubiquitous in solidification and occur in situations from commercial castings to frost on a windshield.

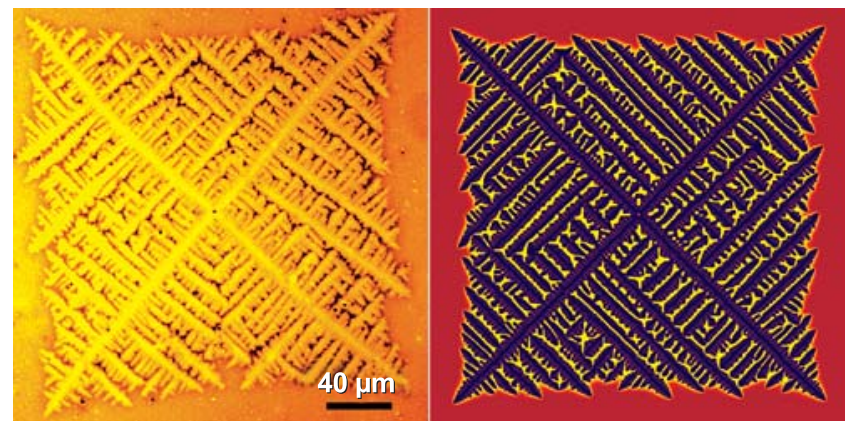

Figure 1: The growing PEO-PMMA polymer dendrite on the left exhibits the four symmetry of the underlying crystal, while the phase field simulation on the right shows striking similarities in its growth form.

Classical studies of solidification consider the growth of a crystal from a nucleus, and, in the absence of other effects, this crystal will have a single crystallographic orientation. Thus, as is shown in Figure 1, symmetry reflecting the underlying four-fold crystal-lattice symmetry is evinced in the growth shape. Indeed, the left figure shows the growth of an undercooled polymer blend (PEO-PMMA) crystallizing in a dendritic pattern with four main branches and a network of fine side branches. The phase field simulation on the right matches this growth form quite well. While the simulations are two-dimensional, the polymer films are thin enough that this is a reasonable approximation.

While such dendritic growth patterns are common, under certain circumstances dendrites can grow which appear to not reflect the underlying crystallographic symmetries, and often, the branches of these dendrites curve in a seemingly erratic fashion. We have termed these growth structures "dizzy dendrites," and two exemplary experimental results are shown in Figures 2a
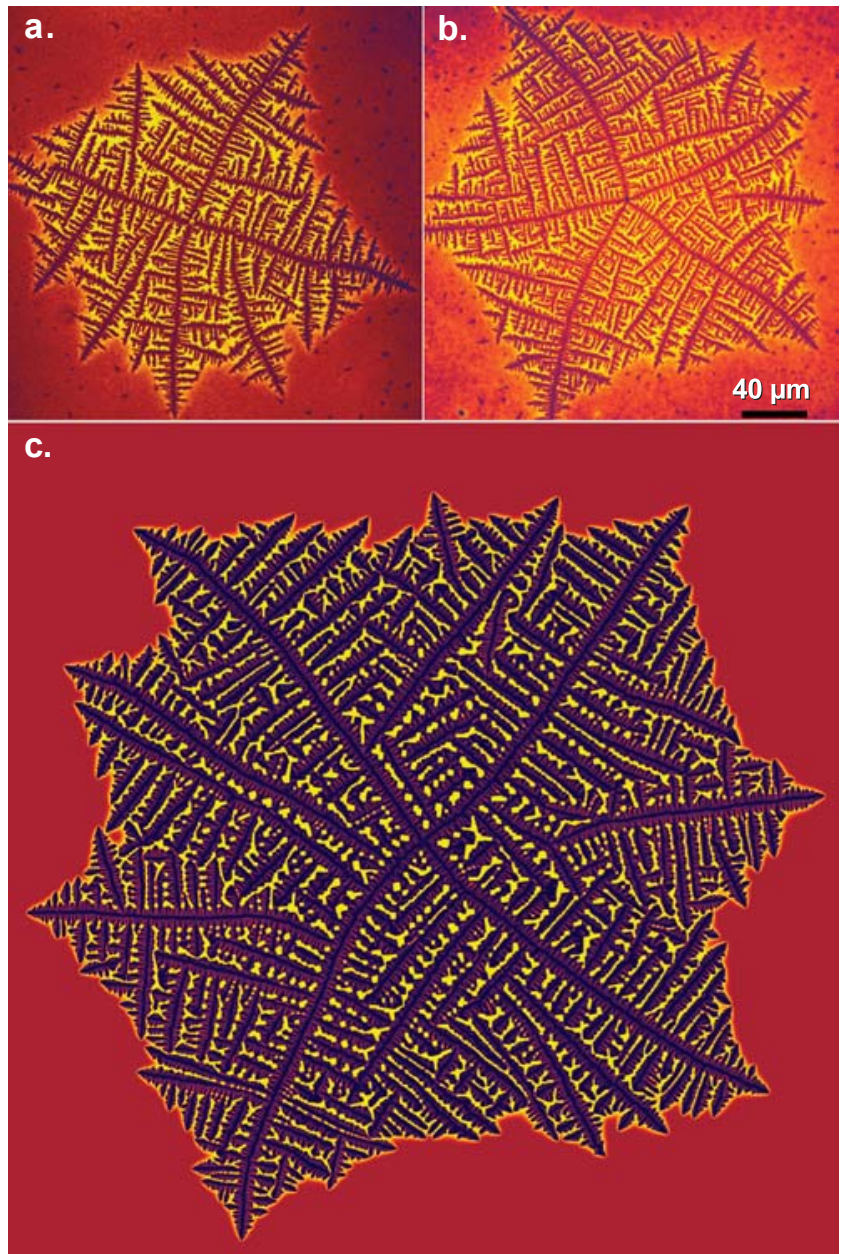

Figure 2: The growing PEO-PMMA polymer dendrites in the upper two images exhibit what we have termed dizzy dendritic growth. The simulation in the lower image is again provocatively similar in its growth form. 
and $2 \mathrm{~b}$. One of the keys to the creation of such growth structures is the presence of clay nucleating agents in the polymer blend, and it has been hypothesized (based on the observation of dendrite tip deflection as it traversed such an impurity) that the presence of these nucleating agents was causing the observed "dizziness."

However, the very fact that the dendrite no longer reflects the symmetry of the underlying crystal immediately requires that the dendrite no longer be a single crystal, and, thus, a more complex phase field model which incorporates multiple grain orientations is required if we are to describe and predict this interesting phenomenon.

Over the past few years, Metallurgy Division scientists, in collaboration with researchers at MIT and Hokkaido University, have developed a phase field model that extends the models of solidification to include polycrystalline growth. As is often the case in scientific research, the ultimate uses of this model are not those necessarily anticipated by the model developers. In this case, researchers at the Research Institute for Solid State Physics and Optics in Budapest collaborated with NIST scientists to harness this model to explain the observed dizzy dendrites.

Specifically, we have postulated the presence of impurities that, upon incorporation into the solidifying crystal, may cause the creation of a grain boundary. We modeled these impurities by assuming a small region of space has a crystal orientation that is fixed (or has a predetermined orientation as a function of time). We refer to these regions of fixed orientation as pinning centers. Among the many interesting observations found during the evaluation of this model, by numerical experiments, we observed that the dendrites would not typically deflect from their original trajectory unless the tip of the growing stalk crossed very near a pinning center. Studies were also made of the sensitivity of the deflection as a function of the size and misorientation of the pinning center. Finally, we considered a random orientation distribution at a density chosen to be the same as the experimental value for clay nucleating agents in the PEO/PMMA experiments shown in Figure 2. When the simulation was performed, the microstructure had a strong resemblance to experimental observations, as is seen in Figure 2c.

Having successfully modeled the growth of this complex pattern, we now are investigating the possibility of using our increased understanding of these systems to control the types of microstructure

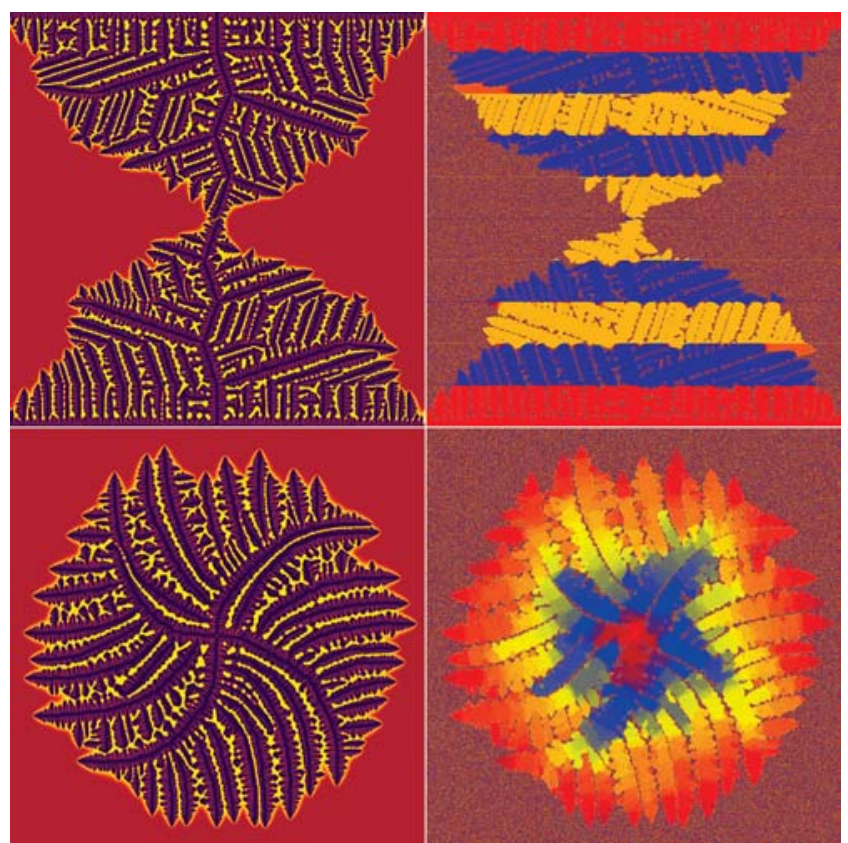

Figure 3: This new phase field model can also be used to predict methods to control microstructural patterns. The patterns on the left indicate concentration of solute, while the patterns on the right indicate the orientation of the crystal. The structures on the top were induced by laying down horizontal lines of fixed orientation. This causes the main stalk to zigzag. In the lower figures, we include orientation pinning centers that spin at a fixed rotation rate, causing the dendrite to develop a pinwheel structure.

that can be created. With that in mind, we explored how the manipulation of included pinning centers can influence the growth shape of dendritic patterns. In Figure 3, we show two such experiments, indicating both the solute distribution (on the left) as well as the crystallographic orientation (on the right). In the upper simulation, we laid down 11 horizontal lines of fixed orientation. This resulted in a main dendrite stalk that zigzagged, while changing orientation to reflect the choices made by the pinning lines. In the lower simulation, we placed a random distribution of pinning centers, all of the same orientation, and insisted that the orientations rotate in time with a fixed frequency. We imagine that a clever experimental setup could realize such control using electromagnetic effects or surface patterning of growth substrates.

\section{For More Information on this Topic}

J. Warren (Metallurgy Division, NIST); Jack Douglas (Polymers Division, NIST); R. Kobayashi (Hokkaido University, Japan); L. Granasy (RISSPO, Hungary); W.C. Carter (MIT) 


\section{Advanced Metallizations for Sub-100 nm Electronics}

Electrodeposited copper is rapidly replacing aluminum for on-chip "wiring" because of its lower electrical resistivity, superior electromigration behavior, and the ability to fill fine features without the formation of seams or voids. As feature dimensions go below $100 \mathrm{~nm}$, difficulties in maintaining performance are anticipated. We are addressing these issues through a combination of modeling and experimental efforts. In FY2003, we have pushed the quantitative limits of the Curvature Enhanced Accelerator Coverage (CEAC) mechanism developed in the Metallurgy Division of NIST. Exploration of the mechanism has yielded new electrochemical processing routes for improved metallization and is guiding the development of advanced metallizations beyond copper as well as new processing routes such as ruthenium barriers for seedless electrodeposition of copper.

\section{Thomas P. Moffat and Daniel Josell}

$\mathrm{T}_{\mathrm{i}}^{\mathrm{h}}$ his project is meeting the microelectronics industry's need for improved device metallizations by exploring advanced materials and process models for superconformal film growth. In FY2003 we have:

- Developed a two-step process for obtaining superfill during silver electrodeposition;

- Demonstrated superconformal feature filling using ruthenium barriers without copper seeds;

- Described an exact solution for feature filling under conditions of fixed total catalyst coverage;

- Extended the CEAC mechanism to explain surface stabilization of planar deposits during deposition of metals in electrolytes containing surfactant additives;

- Quantified consumption of adsorbed catalyst (neglected in previous CEAC models) which can substantially affect feature filling under certain deposition conditions.

\section{Technical Details}

Filling of trenches and vias with silver and copper is quantitatively explained by the Curvature Enhanced Accelerator Coverage Mechanism (CEAC) developed at NIST. Our current measurement and modeling effort is focused on copper and silver metallizations, the latter metal having the lowest resistivity of any element and, thus, having potential on-chip applications.

A two-step process for superfill of high aspect ratio features during silver deposition was demonstrated for the first time. In this process, the catalyst is adsorbed on the patterned wafer prior to metal deposition; in traditional copper processing, a single electrolyte contains both the catalyst and the metal ions. As explained by the CEAC mechanism, this process offers the possibility of improved filling of finer features with higher aspect ratios through minimization of the conformal "incubation" period of growth. In addition, the plating bath requires fewer components and is thus easier to maintain under industrial conditions.

In a review of NIST research by International Sematech in November 2000, Aaron Frank, Program Manager for Copper Deposition, requested that we also examine a growing problem for on-chip interconnects: poor deposition of seed layers as features shrink. In response to this request, we demonstrated in FY 2003 that superconformal electrodeposition directly on ruthenium barrier layers on patterned substrates eliminates the difficulties associated with sputter-depositing copper seeds on tantalum and titanium nitride barriers, again allowing filling of finer features with higher aspect ratios (See Figure 1).
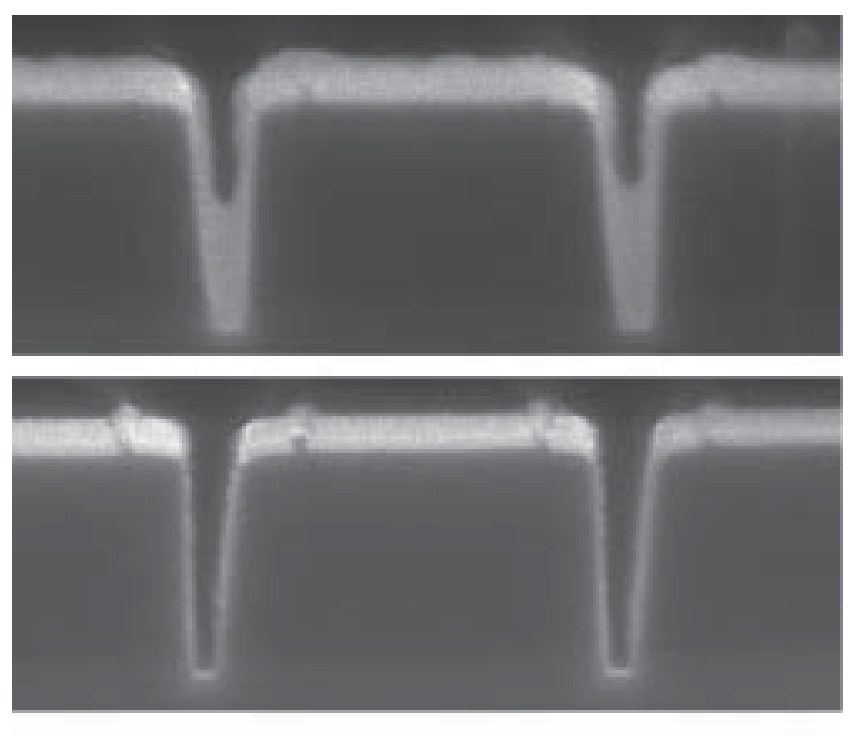

$60 \mathrm{~nm}$

Figure 1: Bottom-up copper fill during electrodeposition in $50 \mathrm{~nm}$ wide trenches with no copper seed is visible in the top image. The patterned dielectric with the ruthenium barrier alone is visible in the bottom image.

Computer codes, that quantitatively predict superconformal deposition in fine features through the CEAC mechanism, evaluate concentration fields in the electrolyte as well as surface coverage of adsorbed catalyst on a moving boundary. Our closed-form solution for fixed initial coverage of catalyst can be used to assess the accuracy of these codes, increasing the reliability of 
their predictions when applied to more complex conditions including catalyst accumulation and consumption.

In a development with application far beyond the microelectronics industry, our recent publication on the CEAC mechanism of brightening is the first to quantitatively explain the impact of adsorbed catalyst on surface stabilization during growth. This work is possibly the most significant advance in understanding surface stabilization since the growth of surface perturbations due to concentration gradients was first described. It is as significant to creating shiny electrodeposits of metal on a car bumper as it is to reducing defect-inducing surface roughness in feature filling for microelectronics.

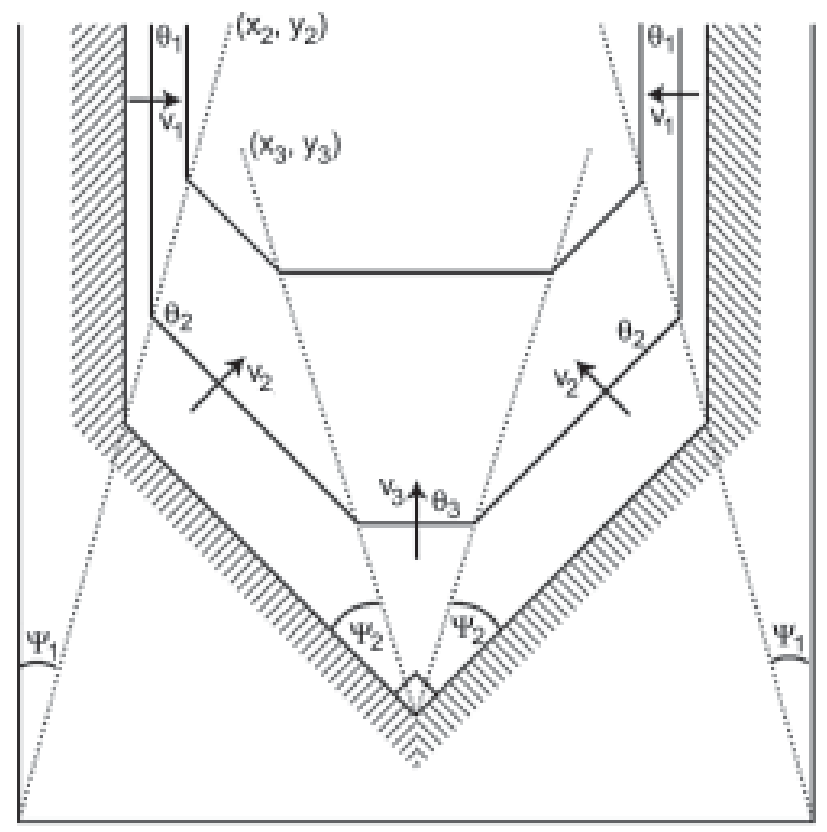

Figure 2: The geometry used to define the exact solution for the superconformal filling of a trench. The metal deposits at the bottom corners of the trench have met in the middle where a new, even more rapidly moving, growth front emanates.

Electrochemical and surface analytical measurements on planar substrates were used in FY2003 to establish rates of catalyst consumption that affect both feature filling and, quite likely, the microstructure of the metal deposits. Previous CEAC-based models have discounted such consumption, and this work demonstrates the reasonableness of such assumptions for the previously studied conditions. It also extends the understanding of CEAC mechanism operation to the less than ideal deposition conditions for which catalyst consumption is significant.

\section{Selected Project Publications for FY2003}

D. Josell, T.P. Moffat, and D. Wheeler. "An Exact, Algebraic Solution for the Incubation Period of Superfill," J. Electrochem. Soc., (in press).
D. Josell, D. Wheeler, C. Witt, and T.P. Moffat. "Seedless Superfill: Copper Electrodeposition in Trenches with Ruthenium Barriers." Electrical and Solid-State Letters, (in press).

G.B. McFadden, S.R. Coriell, T.P. Moffat, D. Josell, D. Wheeler, W. Schwarzacher, and J. Mallett. "A Mechanism for Brightening: Linear Stability Analysis of the Curvature Enhanced Accelerator Coverage Model." J. Electrochem. Soc., (in press).

D. Josell, S. Kim, D. Wheeler, T.P. Moffat, and S.G. Pyo. "Interconnect Fabrication by Superconformal Iodine-Catalyzed Chemical Vapor Deposition of Copper." J. Electrochem. Soc., 150 (5), C368-C373 (2003).

D. Wheeler, D. Josell, and T.P. Moffat. "Modeling Superconformal Electrodeposition Using the Level Set Method." J. Electrochem. Soc., 150 (5), C302-C310 (2003).

B.C. Baker, C. Witt, D. Wheeler, D. Josell, and T.P. Moffat. "Superconformal Silver Deposition Using KSeCN Derivitized Substrates." Electrochemical and Solid-State Letters, 6 (5), C67-C69 (2003).

T.P. Moffat, B. Baker, D. Wheeler, and D. Josell. "Accelerator Aging Effects During Copper Electrodeposition." Electrochemical and Solid-State Letters, 6 (4), C59-C62 (2003).

S.G. Pyo, S. Kim, D. Wheeler, T.P. Moffat, and D. Josell. "Seam-Free Fabrication of Sub-Micrometer Copper Interconnects by Iodine-Catalyzed Chemical Vapor Deposition." J. Appl. Phys., 93 (2), 1257-1261 (2003).

B.C. Baker, M. Freeman, B. Melnick, D. Wheeler, D. Josell, and T.P. Moffat. "Superconformal Electrodeposition of Silver from a $\mathrm{KAg}(\mathrm{CN})_{2}-\mathrm{KCN}-$ KSeCN Electrolyte." J. Electrochem. Soc., 150(2), C61-C66 (2003).

T.P. Moffat, D. Wheeler, C. Witt, and D. Josell. "Superconformal Electrodeposition Using Derivitized Substrates." Electrochemical and Solid-State Letters, 5(12), C110-C112 (2002).

D. Josell, B. Baker, C. Witt, D. Wheeler, and T.P. Moffat. "Via Filling by Electrodeposition: Superconformal Silver and Copper and Conformal Nickel." J. Electrochem. Soc., 149(12), C637-C641 (2002).

Our findings have also been conveyed to the U.S. microelectronics industry, academia, and other national laboratories through more than 15 external presentations in the last year, including an invited presentation at Intel's Portland, Oregon facility. Project publications generated $100+$ citations in the past year.

\section{For More Information on this Topic}

D. Josell, D. Wheeler, T.P. Moffat (Metallurgy Division, NIST) 


\section{High Throughput Data Analysis of Multicomponent Multiphase Diffusion Data}

\begin{abstract}
Multicomponent diffusion processes are part of many complex industrial processes. Reliable data and analysis are critical to effective understanding and development of such processes and the resulting materials. For example, control of multicomponent diffusion is essential for optimizing heat treatments, predicting service life times, and determining optimal material properties. Developing and modeling these processes requires efficient methods to use and store massive amounts of diffusion data as well as improved techniques for understanding multicomponent diffusion data.
\end{abstract}

\section{Carelyn E. Campbell and William J. Boettinger}

\begin{abstract}
$\mathrm{A}^{\mathrm{n}}$ $\mathrm{n}$ improved understanding of multicomponent multiphase diffusion is motivated by two major industrial interactions: the DARPA/General Electric (GE)-led AIM (Advanced Insertion of Materials) program, which highlighted the need for modeling multicomponent diffusion in Ni-base superalloys, and the March 2002 NIST roadmapping workshop on "Databases for Computational Thermodynamics and Diffusion Modeling." As a part of the AIM program, a diffusion mobility database for Ni-base superalloys was developed. An essential resource in developing the diffusion mobility database was the NIST Diffusion Data Center, which catalogs all of the diffusion work between 1950 and 1980. The developed diffusion mobility database was then used in conjunction with the NIST-developed thermodynamic database for Ni-base superalloys to simulate various multicomponent diffusion couples. The diffusion simulations were compared to experimental results provided by GE. Figure 1 shows one such comparison for a Ni/Rene 88 diffusion couple. The experimental and calculated composition profiles are in good agreement, but several questions remain on how to best analyze the data from multicomponent diffusion couple experiments. Current diffusion simulation methods predict the diffusion coefficients as function of composition and time; however, the ability to extract comparable experimental diffusion coefficients from multicomponent diffusion couples is limited.
\end{abstract}

The second industrial interaction motivating further diffusion work was the result of interactions at the March 2002 NIST workshop on "Databases for Computational Thermodynamics and Diffusion Modeling." One of the action items from the 2002 workshop was the development of a diffusion work group to address a variety of multicomponent diffusion questions as well as to promote the development of multicomponent diffusion mobility data.

Based on the needs identified in 2002, a diffusion workshop series began at NIST founded on the following objectives:

- To improve communication among experts in multicomponent diffusion measurement, analysis, and simulation.

- To establish the most efficient method for extracting diffusion data (diffusion coefficients, fluxes, marker location) from multicomponent diffusion couple experiments.

- To provide a forum to solve common diffusion software execution problems.

- To agree on a common diffusion mobility database assessment procedure.

- To establish a general approach to data handling and diffusion modeling in ordered phases.

- To develop standard problems and a website for inter-laboratory comparison of diffusion simulation methods and data extraction techniques.

Following the above objectives, the first of a series of workshops on "The High Throughput Analysis of Multicomponent Multiphase Diffusion Data" was held at NIST, March 27-28, 2003. The workshop format was designed to encourage active discussion among participants and hands-on computer demonstrations.

Presentations on available software for simulating multicomponent diffusion couples included discussions of the DICTRA (Diffusion Controlled Transformation) finite-difference diffusion code and Profiler. The MultiDiflux software code was presented as a tool for determining interdiffusion coefficients from multicomponent data. The development of diffusion mobility databases was presented.

Discussions also included a review of diffusion notation, the advantages and disadvantages of various inverse methods (methods used to determine diffusion coefficients from experimental composition profiles), and a review of various experimental techniques. The group also assessed the limitations of various commonly used methods, including the limits of the Darken approach and the Boltzmann-Matano method. 
Representatives from industry presented experiences in using various diffusion software codes as well as experimental methods. A strong industrial need was articulated for developing the understanding of diffusion in amorphous material, specifically in bulk metallic glasses, and for developing multicomponent diffusion data and modeling in ordered phases.

In the first diffusion workshop, several action items were identified, including the development of a website to post the group's progress and of various tools for teaching multicomponent diffusion. A second diffusion workshop in the fall of 2003 will highlight an introduction to first principle calculations for diffusion, experimental issues in obtaining quality data, and an analysis of multicomponent diffusion in multiphase materials. The complexity associated with multicomponent multiphase diffusion is shown in Figure 2 comparing the experimental and calculated Co composition profiles for the Rene88/IN100 diffusion couple. At $1150{ }^{\circ} \mathrm{C}$, IN100 is a two-phase mixture of $\gamma$ and $\gamma^{\prime}$. While the diffusion simulations consider the two-phase equilibrium during the diffusion simulations, experimental methods for extracting diffusion coefficients from multiphase materials are not well defined.

At both the 2002 NIST Workshop and the 2003 Diffusion Workshop, both industrial and academic diffusion experts described the value of having the NIST Diffusion Data Center converted to a searchable electronic form. The Diffusion Data Center represents a unique collection of diffusion work up until 1980, which is cataloged by author, diffusion system, and selected keywords. The collection contains translations of published works and difficult-to-obtain government reports and theses. To preserve this work and to

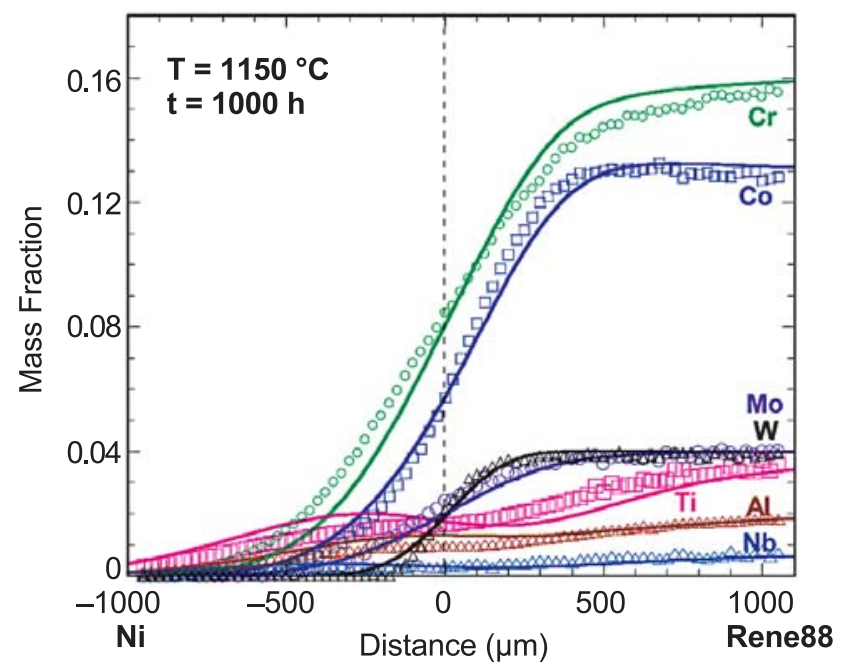

Figure 1: Calculated (solid lines) and experimental (symbols) compositon profiles for the Ni/Rene 88 diffusion couple at $1150{ }^{\circ} \mathrm{C}$ for $1000 \mathrm{~h}$. Experimental profiles from GE-CRD.

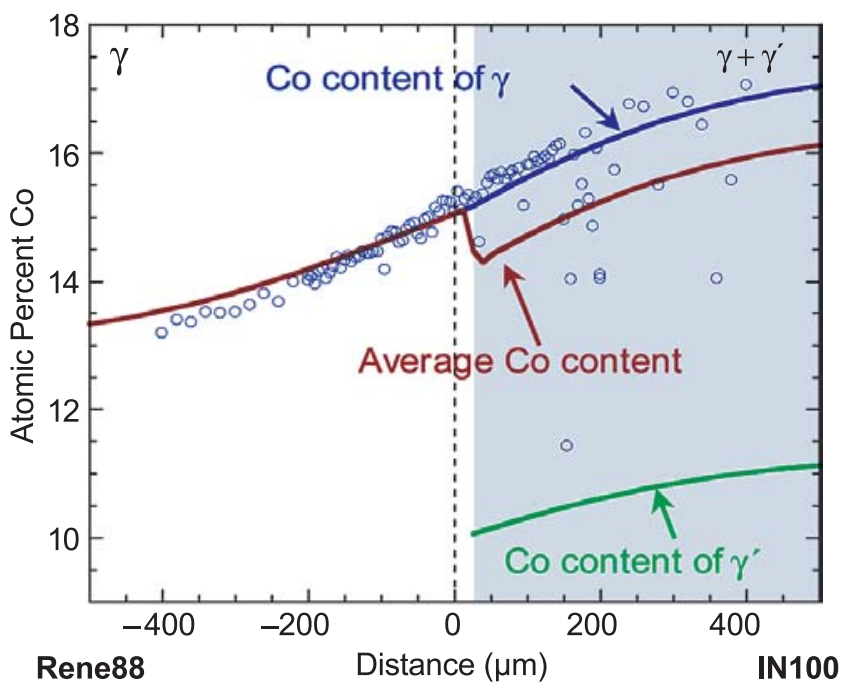

Figure 2: Calculated and measured (symbols) Co composition profiles in the Rene88/IN100 diffusion couple at $1150^{\circ} \mathrm{C}$ for $1000 \mathrm{~h}$. At $1150^{\circ} \mathrm{C}$, IN100 is a two phase mixture of $\gamma+\gamma^{\prime}$. The red line is the calculated average Co profile. The blue line is the Co profile in the rphase and green is the Co profile in the $\gamma^{\prime}$ phase. The light blue region indicates the predicted two-phase region in the couple after $1000 \mathrm{~h}$ at $1150{ }^{\circ} \mathrm{C}$. Experimental data from $G E-C R D$.

make it more widely available, the Metallurgy Division is converting the paper-based catalog to a searchable electronic format. Once the catalog is converted to an electronic format, it will be made available on the NIST website. The work will then focus on converting the difficult-to-find papers, reports, and theses to an electronic format, which can be accessible via the NIST website.

Through the continuing NIST Workshops, action plans for the Diffusion Working Group, and development and application of multicomponent diffusion mobility databases, NIST interaction with academia and industry will continue to better address critical diffusion issues. Ultimately, this increased accessibility to diffusion data will provide material producer and user industries with a variety of tools to more efficiently use and model multicomponent diffusion as a vital part of process modeling.

\section{For More Information on this Topic}

C.E. Campbell, W.J. Boettinger, U.R. Kattner (Metallurgy Division, NIST); J.-C. Zhao (GE-CRD)

C.E. Campbell, W.J. Boettinger, and U.R. Kattner. "The Development of a diffusion mobility database for Ni-base superalloys." Acta Mater, 50, 775-792 (2002).

www.ctcms.nist.gov/ cecamp/workshop.html 


\section{Modeling Dynamics, Damping and Defects for Perpendicular Magnetic Recording Media}

\begin{abstract}
In its accelerating march toward ever higher recording densities, the hard disk drive industry is moving toward perpendicular recording, where the magnetization of the written bits is directed perpendicular to the disk surface. One of the metrology needs generated by the shift to perpendicular recording is the characterization of inhomogeneity in the media. This article describes our development of models that enable measurement of inhomogeneity in perpendicular media by ferromagnetic resonance.
\end{abstract}

\section{Robert D. McMichael}

$\mathrm{O}$ ne of the growing metrology needs of the hard drive industry is the ability to characterize the new generation of perpendicular recording media, where the information is stored as magnetic bits magnetized perpendicular to the disk surface. To store these bits, it is desirable to have media with small, nearly decoupled grains with strong, uniform anisotropy and easy axes directed along the disk normal. Typical grain sizes in the new media are approximately $10 \mathrm{~nm}$.

Characterization of the average properties of perpendicular media is routinely accomplished by magnetometry. However, there is a significant need for information about the inhomogeneity of perpendicular media, particularly the angular easy axis distribution and the distribution of anisotropy fields. Inhomogeneity of the media impacts the bit writing process, analysis of bit stability, and media noise in the read process.

Information about the dispersion of the crystallographic axes can be obtained from x-ray diffraction, but magnetic coupling may force two crystallographically distinct grains to behave as a single magnetic grain, so a purely magnetic measurement of the dispersion angle would be more closely related to the performance of the media.

For variations in the anisotropy field, the only estimates currently available are based on TEM compositional analysis coupled with anisotropy studies on bulk materials. Here again, a direct, purely magnetic measurement would give a clearer indication of the magnetic performance of the media.

The new perpendicular media, with aligned anisotropy axes, is much better suited to ferromagnetic resonance measurements than longitudinal media. Ferromagnetic resonance is a peak in the transverse susceptibility at a frequency that corresponds to precession of the magnetization about its equilibrium orientation. The width of the ferromagnetic resonance peak, or "line width," is the combined effect of damping and inhomogeneity. While there are well-established models for damping, better models of inhomogeneity were needed for interpretation of line width data.

Through our modeling the dynamics of inhomogeneous films, we have laid the theoretical support needed for interpretation of line width data to obtain inhomogeneity information on magnetic thin films.

Previously, there were two known models of ferromagnetic resonance line broadening by inhomogeneity: the "local resonance" model and the "two-magnon" model. The local resonance model assumes that interactions are not important, and the line width is therefore a direct measure of the inhomogeneity strength. The "two-magnon" model includes interactions but, because it is a result of perturbation theory, is limited to weak inhomogeneity. The two-magnon linewidth depends quadratically on the inhomogeneity strength. The models give very different results, but both models have been successfully used to describe separate sets of linewidth data.

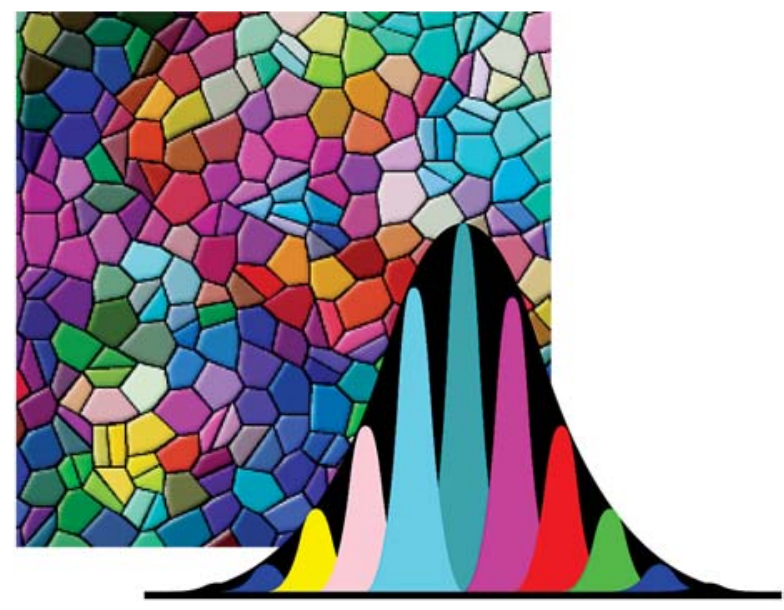

Figure 1: In the absence of interactions, inhomogeneity leads to local resonances at a distribution of frequencies, leading to a broadened resonance line. Interactions cause narrowing of the envelope peak described by the two-magnon model.

To understand the relationship between the local resonance and two-magnon models of line width, we modeled a series of inhomogeneous films with different grain sizes and different levels of inhomogeneity, and we computed the eigenmodes of the magnetization 
motion (Figure 1). We found that the behavior of the magnetization dynamics depends on the relative strengths of the interactions and the inhomogeneity. For a uniform film, the eigenmodes are spin waves ("magnons" in quantum mechanics), and inhomogeneities or strong interactions cause mixing of the spin wave modes in agreement with the two magnon model. For strong inhomogeneities relative to interactions, the dynamics were localized on individual grains in agreement with the local resonance model. These results are summarized in Figure 2, where small grain sizes correspond to strong intergranular interactions, and large grains correspond to weak interactions. These results unify the local resonance and two-magnon models.

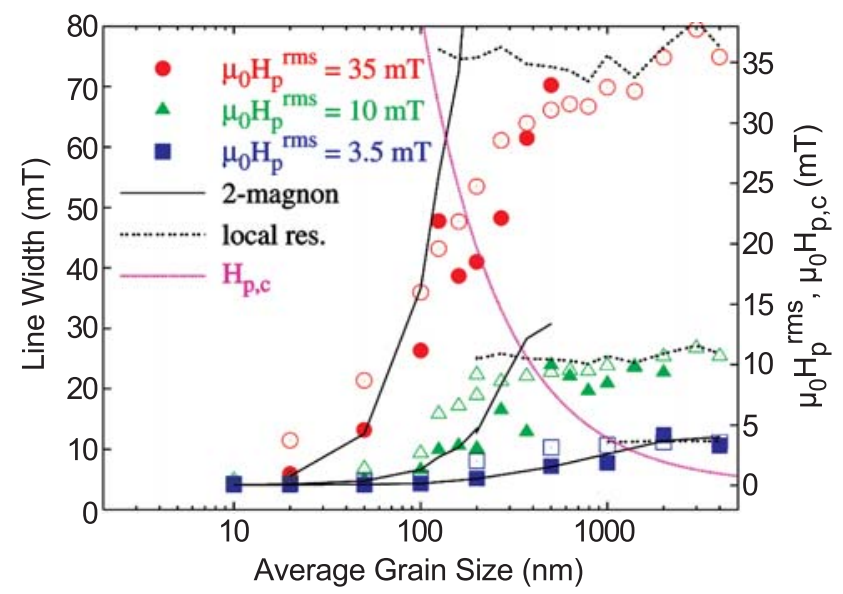

Figure 2: Line widths as a function of grain size in a Permalloy film calculated by eigenmode analysis for three random field strengths $H_{p}$. (Open and closed symbols are for two methods of computing line width from the eigenmode spectrum.) The line width transitions smoothly from the local resonance model for large grains (weak interactions) to the two-magnon model when grains are small. The pink line indicates where interactions and inhomogeneity are roughly equal.

The full eigenmode analysis takes a significant amount of computation time, making it a poorly suited technique for data fitting. However, the results in Figure 2 suggest a simple first approximation; for a given combination of microstructure and inhomogeneity, the lesser of the two-magnon model and the local resonance model is likely to be valid, except in the range where they are similar.

Figures 3 and 4 show two-magnon and local resonance line width calculations for perpendicular media with thickness $15 \mathrm{~nm}$ and grain size $10 \mathrm{~nm}$ with an average anisotropy field of $1.4 \mathrm{~T}(14 \mathrm{kOe})$. In these figures, the top diagram portrays the local resonance result, the green curve is the two-magnon model with dipolar interactions only, and the blue and pink curves are for fractional and full exchange coupling. For the given inhomogeneity strengths, the two magnon model results are expected to be a good approximation to the line width, but for larger values of inhomogeneity, the two magnon results will exceed the local resonance values and the local resonance results would be valid.
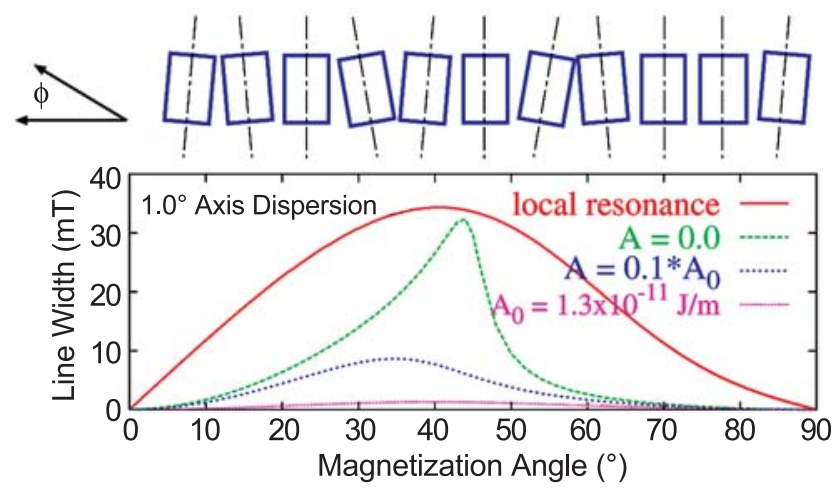

Figure 3: Local resonance and two-magnon line widths resulting from tilting of the easy axis directions in perpendicular recording media with $10 \mathrm{~nm}$ grains. Two magnon results are calculated for three values of exchange coupling.

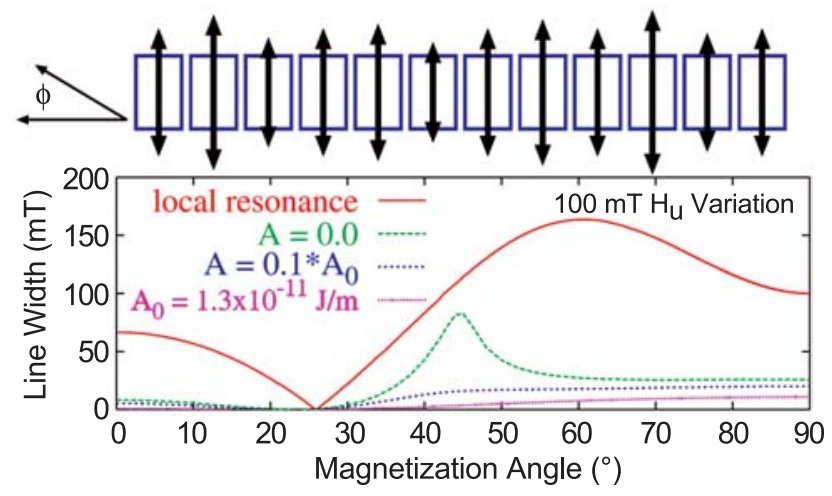

Figure 4: Local resonance and two-magnon line widths resulting from anisotropy variations in perpendicular recording media with $10 \mathrm{~nm}$ grains. Two magnon results are calculated for three values of exchange coupling.

These results show that the ferromagnetic resonance line width can be a sensitive probe of inhomogeneity in perpendicular recording media with the ability to differentiate damping, c-axis dispersion, and anisotropy variations. For weak inhomogeneity, the two-magnon model becomes valid, and the line width can also provide information about exchange coupling.

A ferromagnetic resonance apparatus with the frequency and field ranges necessary to measure perpendicular media is in the design phase.

\section{For More Information on this Topic}

R.D. McMichael, W.F. Egelhoff, Jr. (Metallurgy Division, NIST)

R.D. McMichael, D.J. Twisselmann, and A. Kunz. Phys. Rev. Lett., 90, Art. No. 227601 (2003). 


\section{Artifacts in Ballistic Magnetoresistance Measurements}

\begin{abstract}
Magnetic sensors play a central role in many important technologies ranging from data storage to health care to homeland security. A common need among these technologies is higher sensitivity and smaller size. Advances in the past year in the Ballistic Magnetoresistance (BMR) effect appeared to provide enormous progress in sensitivity in nanoscale devices. The scientific press reported it as one of the major discoveries of the year. Unfortunately, it appears to be wrong. Experiments at NIST have found that several simple artifacts can account for the results. NIST is taking a leading role in designing artifact-free metrology to provide reliable data to the magnetic sensor industry.
\end{abstract}

\section{William F. Egelhoff, Jr.}

$\mathrm{M}$ agnetic sensors play a central role in many important technologies. To name just a few, computer hard disk drives use magnetic sensors to read stored data (Figure 1), magnetoencelophalography uses magnetic sensors to image brain wave activity, and bio-hazard detectors use magnetic sensors to detect magnetic beads bio-tagged for anthrax, botulism, cholera, and other pathogens.

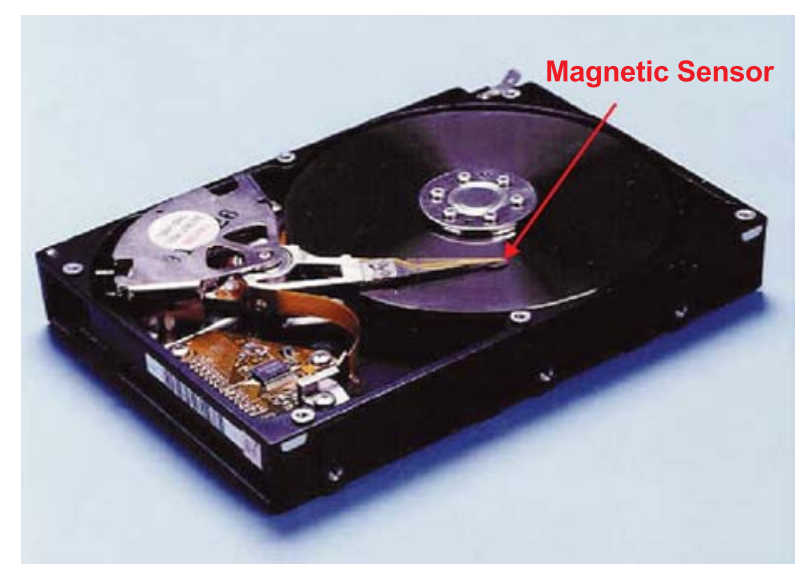

Figure 1: Computer hard disk drives use a magnetic sensor to read data.

A common need among these technologies is higher sensitivity and smaller size. With higher sensitivity and smaller size, more data could be stored on a hard disk, higher resolution imaging of brain activity would be possible, and earlier warnings of bio-contamination could be given.

Currently, the Giant Magnetoresistance (GMR) effect is used for the best small-scale sensors. A GMR sensor can, at best, exhibit $\mathrm{a} \approx 15 \%$ change in electrical resistance in response to a magnetic field. Shock waves went through the magnetic-sensor community in 2003 when a value for the Ballistic Magnetoresistance effect (BMR) of 100,000 \% was published and a value of 1,000,000 \% was reported at a major scientific conference.

Understanding these results suddenly became a high priority for the magnetic-sensor community. For example, Seagate, the world's leading hard-disk drive manufacturer, sent Dr. Erik Svedberg, a physicist in its research labs, to work with us half-time in 2003 to investigate BMR. Seagate selected to work with NIST because we already had a strong program in place to investigate BMR and a network of collaborators that included those groups reporting the high BMR values. NIST had initiated the BMR research program prior to these reports because earlier less-sensational reports of BMR looked promising but faced a number of problems. BMR samples had poor reproducibility, poor stability, and poor reliability. The fabrication techniques were poorly understood, and there was no theory to explain the magnitude of the BMR effect. It was felt that NIST might help with what is often a long, hard road from a laboratory demonstration of a concept to a commercially successful device.

As the NIST program developed over the past two years, it became clear that other labs were able to achieve much higher BMR values, some in the $100 \%$ to $700 \%$ range, while NIST results rarely exceeded $10 \%$. The trend accelerated in 2002 as these other labs began reporting results of $3,000 \%$ to $10,000 \%$. These BMR values were reported as major discoveries in Science, Science News, and Physics News.

In late 2002 and early 2003, scientists from these other labs visited NIST to demonstrate how they achieved these results. NIST staff analyzed the experiments and found them to be subject to a variety of artifacts that can mimic BMR. The small BMR values that NIST had measured were directly attributable to more careful experimental design that excluded the artifacts.

The most common artifact is a result of magnetostriction, in which the length of a magnetic wire changes length in a magnetic field. Figure 2 illustrates the geometry that produced some of the sensational effects attributed to BMR. Two Ni wires are brought together with a gap of $\approx 20 \mu \mathrm{m}$. The gap is bridged by electrodepositing $\mathrm{Ni}$ in a way that forms a nanocontact with one of the Ni wires. This nanocontact is thought to have dimensions on the order of $10 \mathrm{~nm}$. When a magnetic field is applied along the axis of one of the wires, a sharp increase in the electrical resistance 
of the nanocontact is observed, as illustrated in the inset in Figure 2. The results were interpreted as BMR assuming that the magnetic field was magnetizing the two sides of the nanocontact in opposite directions and the resulting magnetic domain wall impeded the flow of electrons, thereby increasing the measured resistance.

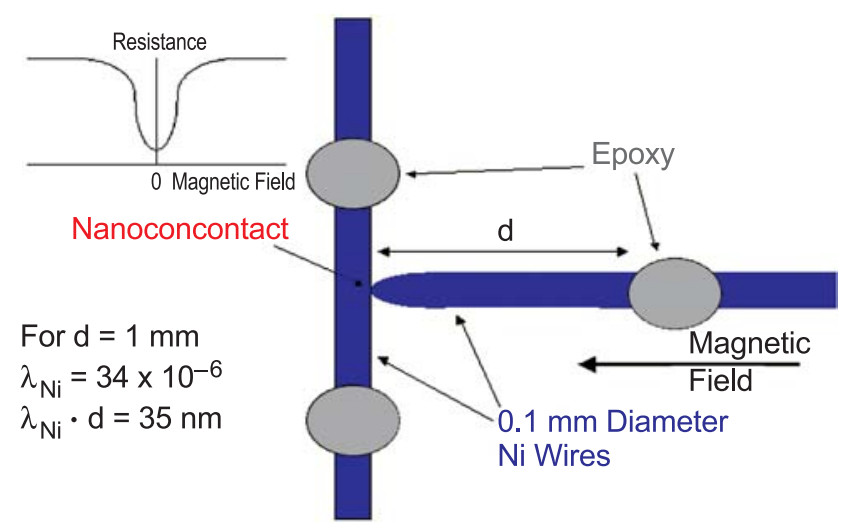

Figure 2: An illustration of the geometry used to generate sensational apparent BMR values.

A simpler explanation accounts for the result: the magnetic field also makes the Ni wire shorter. Using the magnetostriction constant $\lambda$ for $\mathrm{Ni}$ and the length of free wire, the calculated shortening is $35 \mathrm{~nm}$. This distance is enough to stretch and severely deform a $10 \mathrm{~nm}$ nanocontact, increasing its electrical resistance. In fact, in experiments at NIST using electrochemical techniques, it was possible to break and reform nanocontacts thus giving what would correspond to infinite BMR.

Further experiments at NIST using alloy wires whose composition is tailored to have a magnetostriction constant of zero showed no resistance change in a magnetic field. These wires were magnetic and should exhibit BMR if the effect was as claimed, casting further doubt on the high values reported.

Another approach investigated at NIST involved replacing the $\mathrm{Ni}$ wires with $\mathrm{Ni}$ thin films deposited on wafers. The strength of bonding between the $\mathrm{Ni}$ and the wafer anchored the Ni films and suppressed the shortening effect. In this geometry, the nanocontact showed no resistance change, further supporting the conclusion that the high BMR values were not true BMR.

When informed about the NIST results, one of the other labs responded with a method to produce the high BMR values in magnetic nanocontacts electrodeposited at an unusually large voltage on wafers. NIST examined the techniques and found that the unusually large deposition voltage did not produce a solid contact. Instead, the deposit consisted of loosely assembled magnetic particles. When a magnetic field was applied, a compaction of the powder could be observed in a microscope. The small magnetic particles, upon becoming magnetized in the same direction, were clumping together under the magnetostatic attraction, as illustrated in Figure 3.
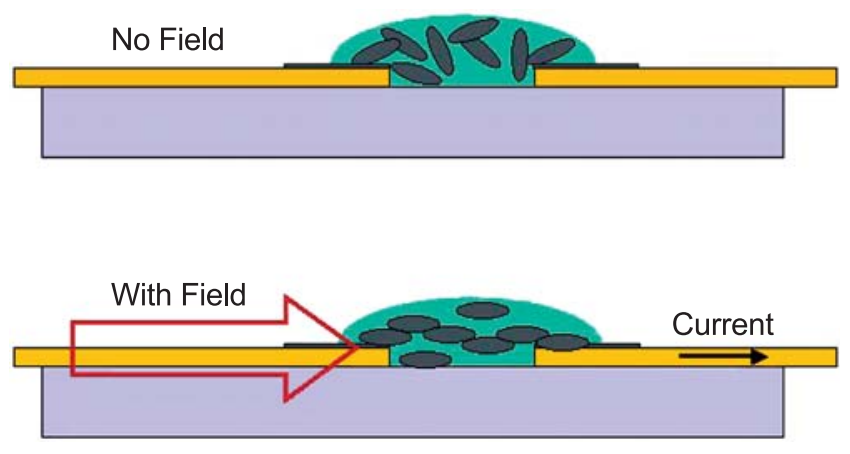

Figure 3: An illustration of how magnetostatic forces cause magnetic particles to clump together to create conducting paths.

In samples made this way, the no-field case was the high resistance state, and the with-field case was the low resistance state. When the particles clumped together, they created additional current-carrying paths to lower the electrical resistance. There was no evidence of anything resembling true BMR in these samples.

A preliminary report of these artifacts was presented at the March Meeting of the American Physical Society in 2003. A more comprehensive report of the work will be presented at the Magnetism \& Magnetic Materials Conference in January 2004.

Experiments are continuing at NIST to put BMR on a firm scientific foundation. Although a conclusive answer is not available at this point, the results, so far, suggest that there may be a small BMR effect but not one that is large enough to be of great interest to the magnetic-sensor community. NIST's work on this topic is likely to be completed early in FY04.

"The technical challenges facing the data storage industry in the next 10 years are so broad and so deep that the industry cannot do it alone. We need researchers like you here at NIST to help us."

\section{Dr. Sining Mao}

Senior Manager, Recording Heads Division, Seagate Technology

\section{For More Information on this Topic}

E. Svedberg (Seagate); N. Garcia (CSIC, Madrid); H. Chopra (SUNY-Buffalo); R. McMichael, T. Moffat, J. Mallett (Metallurgy Division, NIST); M. Stiles (Electron and Optical Physics Division, NIST); C. Powell (Surface and Microanalysis Science Division, NIST) 


\section{Phase Field Modeling of the Electrochemical Interface}

\begin{abstract}
Many important problems in electrochemistry, such as void formation during plating in vias and trenches for on-chip metallization or dendrite formation during recharging of batteries, involve complex topologies that are not easily treated by existing models. The phase field modeling technique has had considerable success studying similar interfaces, with much of the fundamental work performed by researchers in the NIST Metallurgy Division, but the work has almost exclusively focused on solidification. This project seeks to bridge the gap between the traditional electrochemical and phase transformation modeling communities.
\end{abstract}

\section{Jon E. Guyer and William J. Boettinger}

$\mathrm{E}_{\mathrm{t}}^{\mathrm{le}}$ lectrodeposition is increasingly being applied to sub-micrometer features with convoluted morphologies, such as the "copper damascene" process of the micro-electronics industry. In this size regime, factors become important which have been safely neglected in macroscopic models of electrochemistry. For example, as length scales shrink, surface curvature becomes an important consideration; subtle interactions between surface energy and the selective adsorption of different species on the electrode surface can no longer be ignored. Furthermore, traditional models of electrochemical interfaces, such as that of Gouy-Chapman-Stern, take the interface between phases (the transition between electrode and electrolyte) to be abrupt. Such treatments have difficulty tracking a real electrochemical interface, which is often convoluted or can have changing topology, because the boundary conditions on that interface depend on its position, which is part of the unknown solution.

The phase field technique is an alternative approach to modeling phase transformations that has had considerable success in the past decade in the modeling of solidification. The method employs a thermodynamic variable, which is a function of position and time, to describe whether the material is solid or liquid. The behavior of this variable is governed by a partial differential equation that is coupled to the relevant transport equations for the material. The interface between the phases is described by smooth but highly localized changes of this variable. It is not necessary to explicitly track the interface between phases or to apply any boundary conditions there. The method is computationally demanding, but it is powerful because changes in complex interfaces and topology occur naturally.

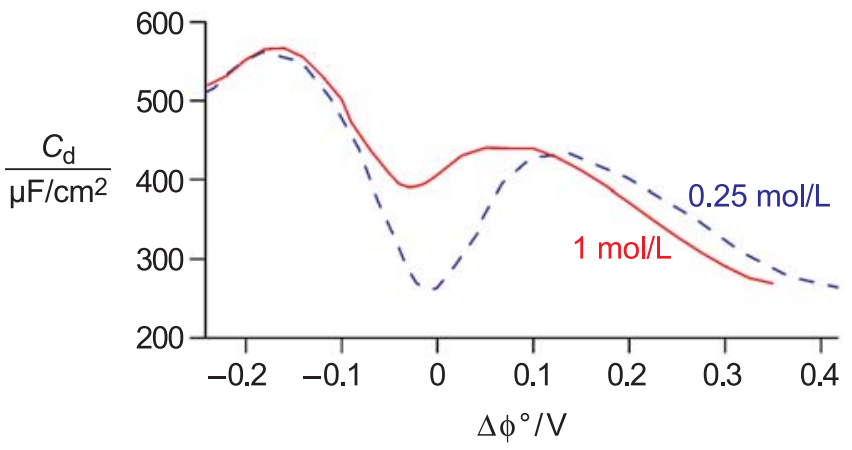

Figure 1: Differential capacitance calculated by our model.

Motivated by the successful phase field models of solidifying interfaces, we seek to expand NIST's "phase field toolkit" into electrochemistry. As a first step to applying the method to convoluted electrochemical interfaces, we have developed a 1D model by making a limited set of assumptions: mass and volume constraints, Poisson's equation, ideal solution thermodynamics in the bulk phases, and a simple description of the competing energies in the electrode/electrolyte interface.

\section{Equilibrium Interface}

The interface between two phases containing free charges is known to develop a charged "double layer." For instance, a layer of excess electrons at the surface of a metal will mirror a layer of excess cations in the adjacent electrolyte. Despite the simple assumptions made in its formulation, our model spontaneously produces just such a double layer. Even though both the total charge and the charge in each bulk phase are zero, we find that there is some non-zero charge distribution in the interface, just as in physical systems. Both electrochemical theory and experiments on inert systems show that the surface charge is directly coupled to the surface energy and that both depend on the potential across the interface. In particular, at the "point of zero charge" (PZC), where the surface charge is zero, the surface energy has a maximum. Independent calculations of surface energy and surface charge using our model exhibit exactly the relationship expected from traditional theories.

The "differential capacitance" is defined by the change in surface charge with potential. The value predicted by classical models of the electrochemical interface depends on the hyperbolic cosine of potential near the PZC and becomes a constant for larger potential differences. The differential capacitance calculated by our model, however, exhibits complex shapes with multiple extrema, as seen in Figure 1. This behavior bears a much stronger resemblance to experimental measurements, such as in Figure 2, than to previous theories. 


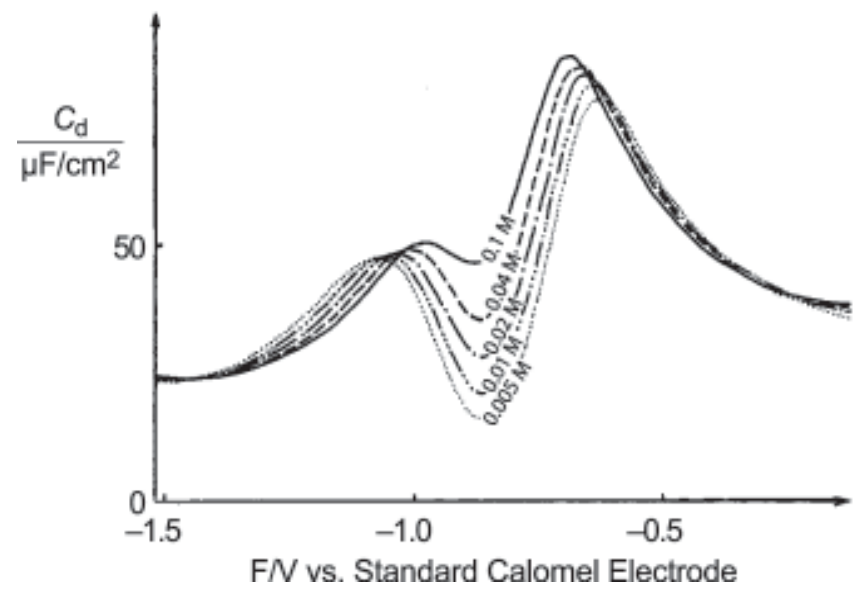

Figure 2: Experimental measurements of differential capacitance on $\mathrm{Ag}(100)$ electrodes in aqueous solutions of NaF. [Reprinted from J. Electroanal. Chem. 138, G. Valette (1982) 37-54), with permission from Elsevier.]

\section{Dynamic Interface}

Turning now to the examination of electrodeposition and electrodissolution, when the overpotential (the deviation from the equilibrium potential difference across the interface) is not zero, current will flow and the interface will move. The classical description for the relationship between current and overpotential is the empirical Butler-Volmer relation (Figure 3).

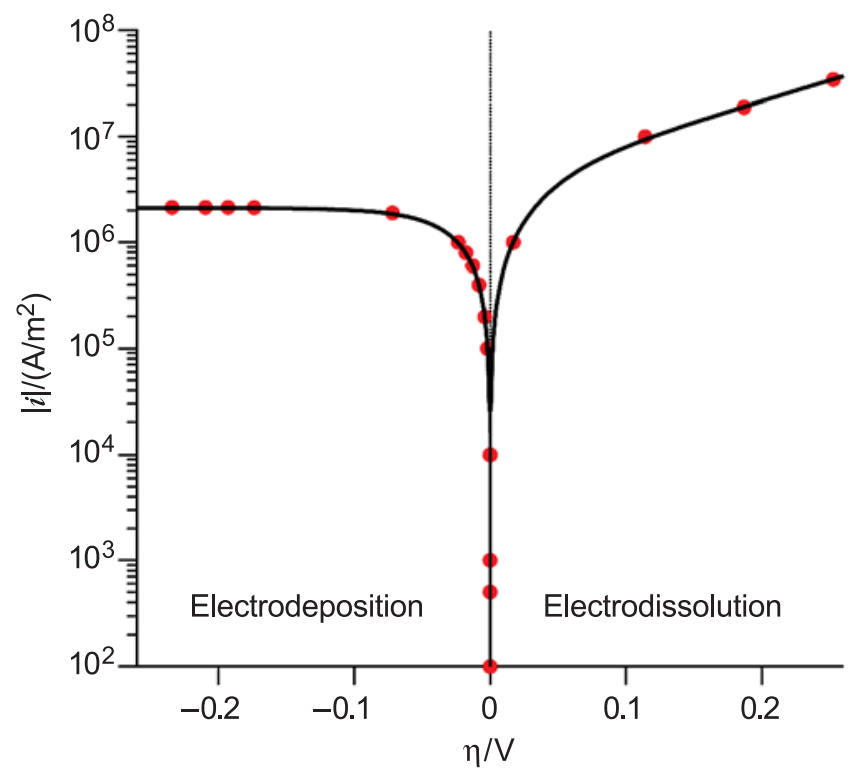

Figure 3: Current vs. overpotential calculated by our model (symbols) and predicted by classical Butler-Volmer equation (line).

In contrast, phase field models typically assume that the rate of change of the phase field is directly proportional to some driving force. Despite making such a simple assumption about the kinetic behavior, the rich interplay among composition, charge, electrostatic potential, and thermodynamic stability gives rise to current-overpotential behavior that agrees very well with the predictions of traditional electrochemistry (Figure 3).

At large overpotentials, we find that the most electroactive cation becomes depleted at the interface nearly to zero. The concentration gradient can be no steeper, limiting the rate of cation diffusion to the interface. In order to carry more current than this diffusion-limited value, the charge structure of the interface shifts to allow deposition of less electroactive species. Figure 4 shows two different times after the current has been changed from $0.005 \%$ to $500 \%$ of the limiting current for the deposition of $\mathrm{M}$, leading to alloy deposition of $\mathrm{M}$ and $\mathrm{N}$.
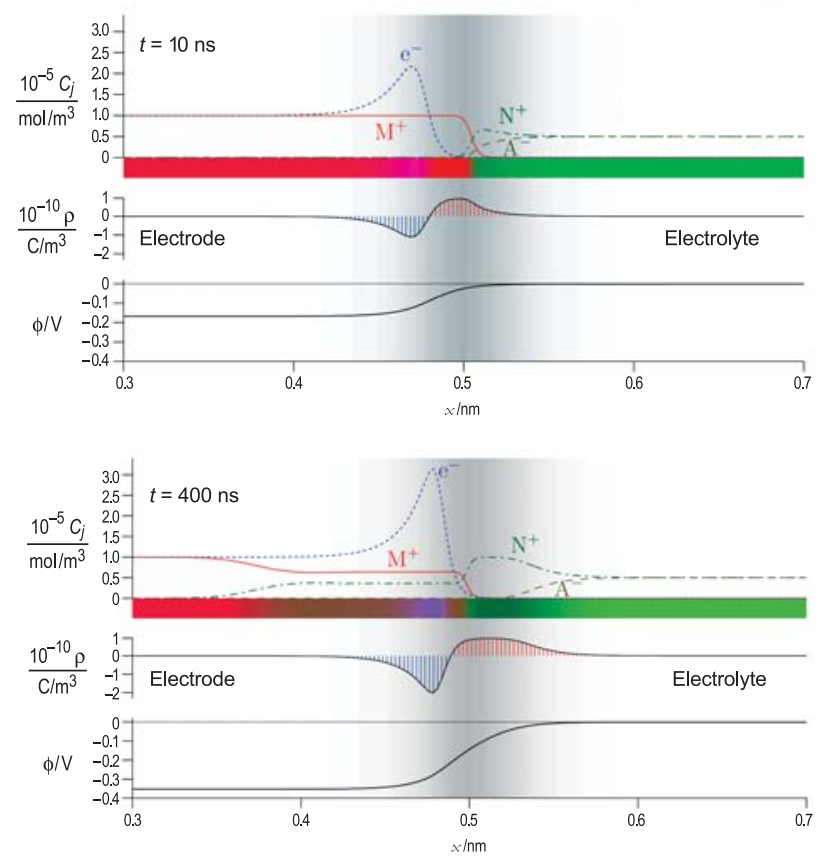

Figure 4: Profiles of the ion concentrations, charge density, and electrostatic potential at two different times after the current was changed from $10^{2} \mathrm{~A} / \mathrm{m}^{2}$ to $10^{7} \mathrm{~A} / \mathrm{m}^{2}$. The gray band indicates the position of the diffuse phase field interface.

By correctly describing the fundamental physics, our model spontaneously produces differential capacitance curves strongly reminiscent of experiments, nonlinear current-overpotential behavior, and alloy deposition, all without making ad hoc assumptions. Future work will focus on further relating the parameters of the phase field model with those of traditional electrochemistry, examining larger physical domains, and to extending the model to two dimensions.

\section{For More Information on this Topic}

J. Guyer, W. Boettinger, J. Warren (Metallurgy Division, NIST); G. McFadden (Mathematical and Computational Sciences Division, NIST) 
NEMI-NIST-TMS Workshop on Tin Whiskers: Cause and Effect

\begin{abstract}
This NEMI-NIST-TMS workshop brought together key researchers to compare in detail research that has been done in the area of tin whiskers, and to see if a consensus could be developed on a basic model defining the conditions for whisker formation, or, if not, on the work required to reach such a consensus. The end goal is to provide direction to the electronics industry in developing deposition processes that will assure that long-lifecycle products will not experience catastrophic failure due to whisker growth.
\end{abstract}

\section{William J. Boettinger and Maureen Williams}

Since the mid 1940s, the phenomenon of metallic $\checkmark$ growths from electrodeposited surface finishes on electronic components has been observed and studied. Electrodeposited surface finishes applied to the component leadframe protect the material from corrosion and improve solderability of the components prior to board assembly. These metallic growths are referred to as whiskers and may form different shapes, as seen in Figure 1.
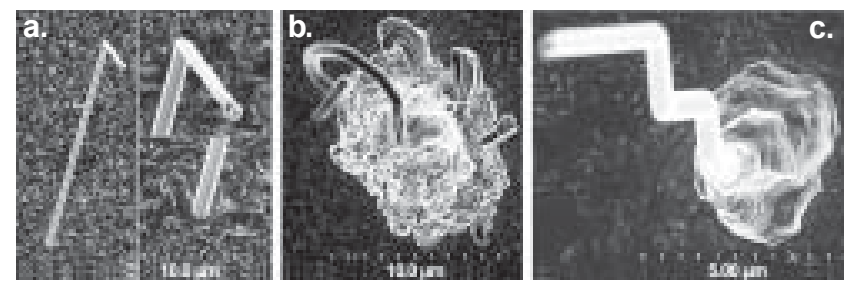

Figure 1: Three different whisker types from bright Sn coatings; a) Filament with tip and base shown in insert; b) Odd shaped eruption with filimenery growths; c) filament growing from an eruption.

The most commonly used commercial surface finishes are Tin (Sn) based. Sn whiskers (filament type whiskers are typically $1 \mu \mathrm{m}$ diameter and several $\mathrm{mm}$ long) can grow from the Sn plate and cause electrical shorts and failures. Tin-lead $(\mathrm{Pb})$ alloys have been used extensively for surface finishing of electronic components because $\mathrm{Pb}$ was found to be effective in retarding $\mathrm{Sn}$ whisker growth and keeping production costs low. Since $\mathrm{Pb}$-free solder alloys have been introduced, electronic manufacturers are seeking a new surface finish technology that will prevent $\mathrm{Sn}$ whisker growth without the use of $\mathrm{Pb}$. Whiskers are generally believed to grow to relieve residual stress in electroplated Sn. Figure 2 shows a schematic of compressive stresses in electrodeposited Sn plating resulting in the growth of a $\mathrm{Sn}$ whisker. Some of the key factors that have been found to influence $\mathrm{Sn}$ whisker growth are: deposit thickness, diffusion, thermal stresses, substrate material, electrolyte type, plating conditions, temperature, humidity, organic and inorganic inclusions, grain size and shape, and interdiffusion barriers. In spite of this wealth of information, a basic understanding of whisker growth and its prevention does not currently exist.

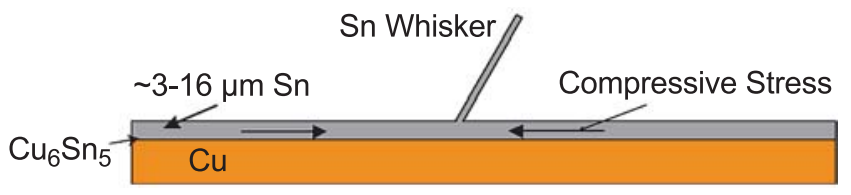

Figure 2: Schematic of Sn whisker growth from a $S n$ electrodeposited plating.

The National Institute of Standards and Technology (NIST), the National Electronics Manufacturing Initiative (NEMI), and The Minerals, Metals \& Materials Society (TMS) co-sponsored a one-day workshop, "Tin Whiskers: Cause and Effect," on Sunday, March 2, at the San Diego Convention Center. The event was held in conjunction with the 2003 TMS Annual Conference and Exhibition.

The agenda consisted of four presentations during the morning and round table discussions in the afternoon. The 44 attendees represented a variety of industrial, academic, and government organizations, including Analog Devices, Brocade Communications Systems, Cookson Electronics, Hanyang University, HP, IBM, Intel, KAIST, Kyocera, Los Alamos National Lab, Michigan State, Millenium Chemicals, Motorola, Oak Ridge National Lab, Research in Motion, Sandia National Laboratory, Shipley TI, Sun Yat-Sen University (Taiwan), SUNY at Buffalo, Technic, The Aerospace Corporation, University of Kentucky, and Washington State University. All of the participants contributed to a lively afternoon discussion of the papers presented and current theories.

\section{Stress in Electroplated Sn}

Dr. Chen Xu of Cookson Electronics outlined his work on understanding the whisker growth rate and carrying out structural and chemical characterizations of whisker growths. He presented a "whisker index" - a proposal to characterize whiskers and presented data on this factor as a function of various types of Sn plating. He discussed the use of x-ray diffraction (XRD) for measuring stress in Sn plated films, showing the changes in stress as a function of time. His data shows that compressive stress in the film leads to whisker growth, while tensile stress does not. 
He presented data to show that a nickel (Ni) under layer will result in tensile stress in the Sn layer, and he saw no whisker growth when a Ni layer was deposited between the $\mathrm{Cu}$ and $\mathrm{Sn}$ layers.

\section{Spontaneous Growth of Tin Whiskers}

Dr. Dong Nyung Lee of the School of Materials Science and Engineering, Seoul National University showed results of microstructure characterization using SEM techniques, including angle of growth, and pole figures. To release the compressive stress in the tin film, tin whiskers grow from the grain whose surface oxide is sheared. The whisker growth is controlled by the expansion of prismatic dislocation loop on the slip plane by climb, that is, by means of the operation of the Bardeen-Herring dislocation source. Whisker growth continues until the stress is relieved.

\section{Tin-Whisker Microstructural Analysis}

Dr. George T. Galyon of the IBM Server Group provided a critical analysis of the literature outlined in his Annotated Sn Whisker Bibliography, noting that there appear to be necessary conditions for whisker formation (i.e., stress) that are not always sufficient to explain the overall phenomena. He provided a great deal of information on the use of the Focused Ion Beam (FIB) tool in examining whiskers. Very little, and only recent, work has been reported using this new tool, and it seems to provide considerable additional insight into whisker structures. His observations indicate that whisker growth is the result of a recrystallization of the tin at the base of the whisker resulting in additional material pushing the whisker out of the film.

\section{Focused Ion Beam, Transmission Electron Microscopy, and Synchrotron Radiation}

Dr. King N. Tu, of the Department of Materials Science and Engineering at UCLA, performed studies on tin whiskers with focused ion beam (FIB) imaging, cross-section transmission electron microscopy (XTEM) observation, and synchrotron radiation (SR) micro-diffraction analysis. Morphology of whiskers and orientation relationship between a whisker and grains in the tin plating has been studied by FIB and XTEM. A large amount of precipitates in the grain boundaries were observed. The precipitation of $\mathrm{Cu}_{6} \mathrm{Sn}_{5}$ intermetallic is believed to maintain the driving force for spontaneous whisker growth.

\section{Discussion Summary}

Discussions established that $\mathrm{Sn}$ whiskers are caused by compressive stress in the Sn plated film, and that material somehow moves through the grain boundaries to form a whisker. Whiskers grow from material deposited at the base of the whisker. The formation of $\mathrm{Cu}_{6} \mathrm{Sn}_{5}$ intermetallics in the $\mathrm{Sn}$ grain boundaries may contribute to the growth of a compressive stress. The whisker may take advantage of weakness in the oxide film to erupt.

While no specific mechanism for Sn whisker growth was established, several questions were posed. Some of these questions included:

- Why is recrystallization of Sn necessary for whisker growth?

- What is the mechanism by which the $\mathrm{Sn}$ is transported to the whisker base?

Discussions also determined several areas that require further study to determine the growth mechanism:

- The role of $\mathrm{Pb}$ in suppressing whisker formation; the role of a surface oxide layer;

- The role of texture;

- The role of impurities; and

- The role of a diffusion barrier.

An understanding of the details of the nucleation process is also needed. The most important conclusion was that further systematic experimental work must be done to establish the growth mechanism.

Currently at NIST, efforts are focused on analyzing the average stress relaxation in electrodeposited films as function of time. These experiments concentrate on in situ stress measurements during the plating process and post-plating. Additional experimental work is planned in collaboration with NEMI members.

"I consider your [NIST Metallurgy Division] group a key asset to industry in making these decisions on the soundest possible scientific basis. There is no comparable body of expertise anywhere in the world that matches that found in your group."

Dr. George T. Galyon (IBM) - Chairman, NEMI TinWhisker Modeling Committee

\section{For More Information on this Topic}

W.J. Boettinger, M. Williams (Metallurgy Division, NIST); R. Gedney (NEMI); K.-W. Moon (University of Maryland) 


\section{Kolsky Bar: Materials Properties at High Strain Rates and High Heating Rates}

\begin{abstract}
Materials processed in industry are frequently placed simultaneously under applied high strain and heating rates. Understanding, predicting, and simulating these processes require databases for materials properties and constitutive equations verified under similar conditions. NIST has responded to these industrial needs by developing a unique testing facility for measuring the needed data and validating the appropriate material models under controlled, high rates of straining and heating.
\end{abstract}

\section{Richard Fields and Debasis Basak}

$\mathrm{M}$ achining processes such as turning, milling, drilling, broaching, etc., are the most commonly used methods of producing precision metal parts. Efficient machining requires a thorough knowledge of the machine/tool/workpiece interactions. This includes understanding material behavior under the conditions of machining. Furthermore, competition has pushed industry to machine parts as rapidly as possible. This has led to the development of codes that predict machining behavior and guide the development of the most efficient machining practices. These codes require, as input, mechanical property data that is not commonly available for most alloys of commercial interest.

At the tool/workpiece interface, the material being removed is subject to extremely high forces and temperatures. The high temperatures arise from the work dissipated by the large and rapid straining. They also result from the friction between the tool and the workpiece material. It has been estimated that the straining occurs at a rate between $10^{3} \mathrm{~s}^{-1}$ and $10^{6} \mathrm{~s}^{-1}$, while the heating takes place at rates up to $10^{6} \mathrm{~K} / \mathrm{s}$. The rapid heating is important because most microstructural features cannot change to their equilibrium structure in such a short time. Previous attempts at collecting high temperature data elsewhere have relied on low rates of heating and thus measured the properties of inappropriate microstructures. It is the controlled, high heating rate that makes the current facility unique.

The facility is built around a conventional Kolsky Bar (sometimes called a Split Hopkinson Pressure Bar) shown in Figure 1. An air gun fires a striker bar at speeds up to $50 \mathrm{~m} / \mathrm{s}$ at the incident bar. On impact, the striker bar generates a roughly square elastic wave in the incident bar twice the length of the striker bar and traveling at nearly $5000 \mathrm{~m} / \mathrm{s}$. This wave propagates down the incident bar to the sample and compresses the sample between the incident bar and the transmitted bar. The elastic strain at any time introduced into the transmitted bar during this process is proportional to the stress in the sample at that time and the elastic strain reflected back into the incident bar is proportional to the strain rate experienced by the sample at that time. By analyzing the strain signal records, the stress/strain behavior of the sample material can be determined under controlled high strain rates.

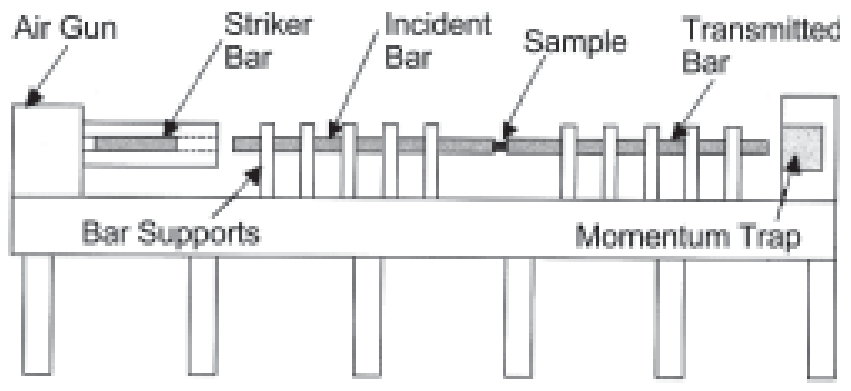

Figure 1a: Schematic diagram of Kolsky Bar apparatus.

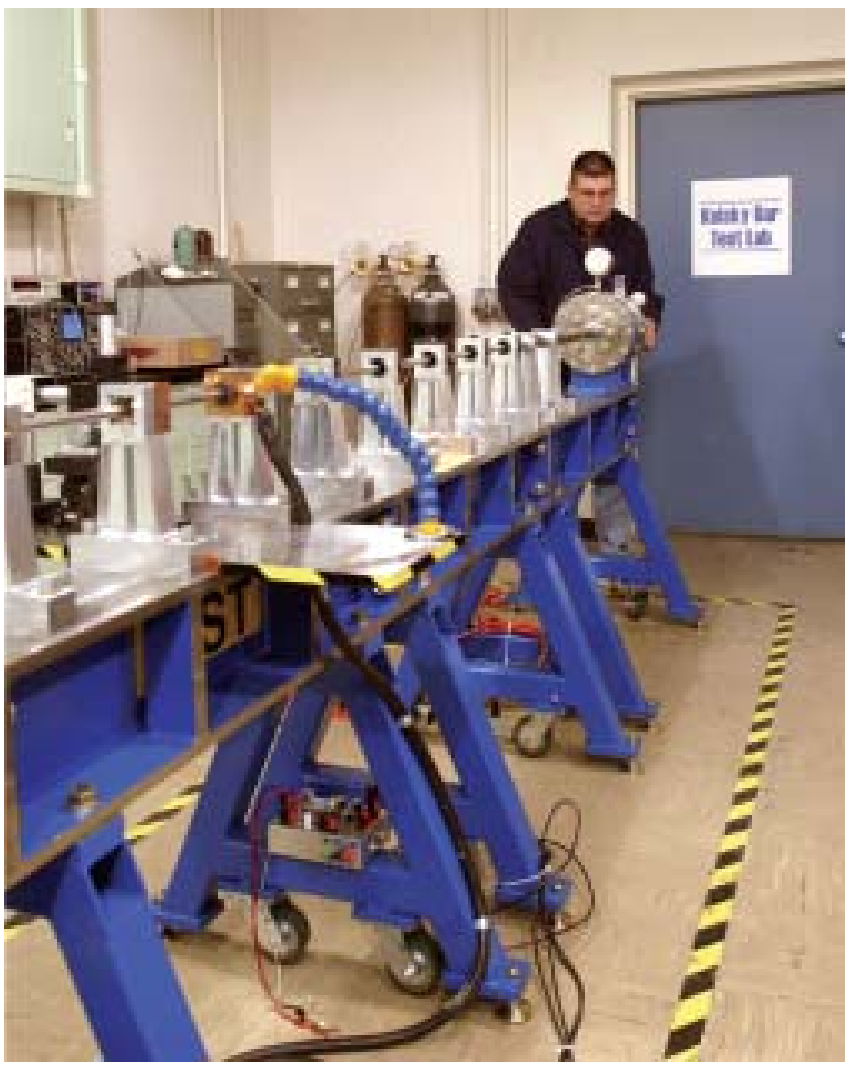

Figure 1b: Photograph of NIST's Kolsky Bar facility. 
A millisecond-resolution pulse heating facility, which relies on rapid resistive "Joule heating" of the specimen by the passage of an electric current pulse of sub-second duration, is used to heat the specimen. The connections for the current input can be seen in Figure 2. Two high-speed pyrometers are used simultaneously during the test. A spot pyrometer measures the specimen temperature with microsecondresolution, and an imaging pyrometer records the temperature distribution in the specimen during the test. The output from the spot pyrometer is also used to control the specimen temperature. When the desired temperature is reached, the electric current to the specimen is either automatically turned off or a feedback control program is activated to achieve isothermal conditions in the specimen, prior to impact.

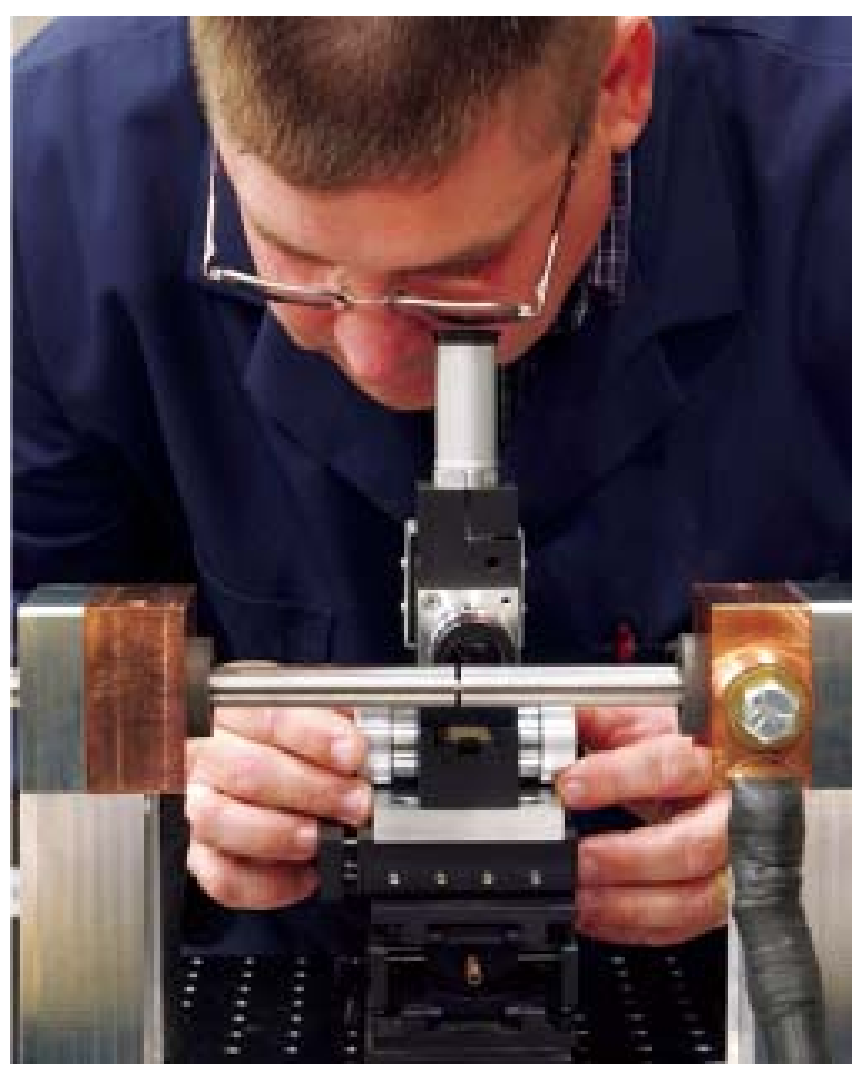

Figure 2: The current for heating is introduced through copper blocks and graphite bearings to the incident and transmitted bars, and then through the sample seen in the middle. The sample temperature is monitored by the pyrometer being focused on it.

This facility was developed to provide insight and data for high speed machining of commercial alloys. To date, it has been used mainly for copper, 303 stainless steel, AISI 1045 steel, and 7075 aluminum alloy. The results of this research are described under "High Speed Machining" elsewhere in this annual report. The data are being provided to industry and other alloys of commercial interest are being investigated.
More than a dozen papers and talks have been presented on this topic in the three years that this project has been underway. Additional papers are being drafted.

Since the facility was developed, other applications for it have arisen. The World Trade Center Twin Tower collapses were initiated by the impact of two commercial airliners. NIST has undertaken the modeling of these impacts to more fully understand the events leading up to the failure of the towers. The models require high strain-rate mechanical properties. The Kolsky Bar facility is providing the rate dependence of plastic deformation for the many types of structural steel in the buildings.

Another application concerns the behavior of projectiles such as those used as military tank penetrators. It has been found that the Kolsky Bar test is one of the best predictors of thermal localization of deformation and the self-sharpening behavior desired in penetrator materials. The Kolsky Bar facility is providing a project in the Metallurgy Division that is investigating new penetrator materials with a rapid assessment of whether their materials or processing routes are improving penetration behavior.

Lastly, considering the significant number of conventional Kolsky bars in use in the U.S., an effort has been initiated to compare the consistency of measurements made with these instruments. To date, NIST has compared its results on 1045 steel and copper with similar results at Aberdeen Proving Grounds and Los Alamos National Laboratory. Such intercomparisons will help improve and maintain consistency of dynamic materials properties measurements.

New applications for this unique facility are being pursued in the areas of dynamic fracture and crack arrest of high strength pipeline steels, bullets and body armor for the Office of Law Enforcement Standards, and additional issues in manufacturing. Since certain types of explosions involve intense, rapid, radiative heating followed by a shock wave, it is felt that the new Kolsky Bar facility may play an important role in research relating to homeland security.

\section{For More Information on this Topic}

Richard Fields, L. Levine, R. deWit (Metallurgy Division, NIST); R. Rhorer, R. Polvani, K. Jurrens, R. Ivester, H. Soons, M. Kennedy, B. Dutterer, (Manufacturing Metrology Division, NIST); T. Burns (Mathematical and Computational Sciences Division, NIST); H. Yoon (Optical Technology Division, NIST); Debasis Basak (University of Tennessee); M. Davies (University of North Carolina, Charlotte) 


\section{Advanced Manufacturing Methods}

The industrial competitiveness of U.S. manufacturers depends on their ability to reduce the time from product concept to market while decreasing product cost. This is as true for well-established "commodity" industries, such as automotive and aerospace, as it is for rapidly growing or emerging industries, such as biotechnology and nanotechnology. Manufacturing is a critical step in realizing such reductions in product cycle time: for many industries and products, the rapid, low-cost development of manufacturing processes is needed to create new materials with complex product shapes and higher performance at the same or lower cost as the competing, older materials and methods.

To realize such improvements in manufacturing, MSEL is working to develop robust measurement methods, standards, and materials and process data that will help in the design, monitoring, and control of manufacturing processes. An increasingly important step in manufacturing is process modeling. Development of optimized manufacturing processes, such as stamping of complex auto panels or machining of large aircraft components, is time consuming and labor intensive. In many cases, the resulting processes are far from optimal because materials variability, caused, for example, by residual stresses or strain localization during processing, leads to lower yields. Our approach is to develop and combine higher precision, spatially localized measurement methods with realistic, physics-based modeling of manufacturing processes and materials process data to create more useful process modeling tools.

Depending on the stage of the research, our research is often conducted in close collaboration with industrial consortia and standards organizations. This close working relationship developed through these organizations not only ensures the relevance of our research, but also promotes rapid transfer of our research to industry for implementation.

The performance of metallic components in products is strongly dependent on processing conditions that determine microstructural features, such as grain size and shape, texture, the distribution of crystalline phases, macro- and microsegregation, and defect structure and distribution. NIST expertise is applied from a wide range of disciplines, including thermodynamics, electrochemistry, fluid mechanics, diffusion, x-ray, and thermal analysis, to develop measurement methods and understand the influence of processing steps for industries as diverse as automotive, aerospace, microelectronics, and defense. Two major MSEL efforts in Polymer Processing and Forming of Lightweight Metals are centered on unique measurement facilities. Rapidly growing and emerging industries such as biotechnology and nanotechnology are also dependent upon the development of new advanced manufacturing methods that can produce metallic components with the desired characteristics and performance. Related projects with significant manufacturing processes components can be found in the MSEL Programs on Nanocharacterization, Materials for Micro- and Opto-Electronics, and the Interface of Materials with Biology.

\section{Forming of Lightweight Metals}

Automobile manufacturing is a materials intensive industry that involves about $10 \%$ of the U.S. workforce. In spite of the use of the most advanced, cost-effective technologies, this globally competitive industry still has productivity issues related to measurement science and data. Chief among these is the difficulty encountered in die manufacture for sheet metal forming. In the ATP-sponsored workshop (The Road Ahead, June 20-22, 2000), the main obstacle to reducing the time between accepting a new design and actual production of parts was identified as producing working die sets. To benefit from the weight saving of high strength steel and aluminum alloys, a whole new level of formability measurement methods and data is needed, together with a better understanding of the physics behind metal deformation. MSEL is working to fill this need.

\section{Polymers Processing}

Polymers have become ubiquitous in the modern economy because of their ease of processing, high functionality, and low cost. However, these materials can exhibit complex and sometimes catastrophic responses to the forces imposed during manufacturing, thereby limiting processing rates and the ability to predict ultimate properties. The focus of our polymer research is on microscale processing, modeling of processing instabilities, and on-line process monitoring of polymers. Our unique extrusion visualization facility combines in-line microscopy and light scattering for the study of polymer blends, extrusion instabilities, and the action of additives. Current applications focus on understanding and controlling the "sharkskin instability" in polymer extrusion and observation of the dielectric properties of polymer nanocomposites. Fluorescence techniques are developed to measure critical process parameters such as polymer temperature and orientation that were hitherto inaccessible. These measurements are carried out in close collaboration with interested industrial partners.

Contact: Carol A. Handwerker 


\section{Anelasticity and Springback Predictability}

Increased computing power and finite element modeling methods have brought the prediction of springback and springback-compensated die design within reach of industry. In developing this technology, industry has identified areas where better measurement methods and data are required, and in some cases, where scientific understanding of materials behavior is incomplete. One such case is the influence of plastic deformation on mechanisms of anelastic recovery and springback. The objective of this project is to develop fundamental understanding, test methods, and data required to predict this phenomenon.

\section{Richard Ricker}

\begin{abstract}
A U.S. Council for Automotive Research (USCAR) Consortium on Springback Prediction has developed finite element springback prediction models to the point where they are considering using these models to guide the design of stamping dies. However, current models have consistently under-predicted springback leading to the inclusion into the models of arbitrary correction factors determined from stamping trials. The systematic and alloy dependent nature of these errors indicate that the materials property data used in making these predictions may be responsible. Preliminary experiments in FY 2002 indicated that this issue warranted further investigation by NIST using a dynamic thermal-mechanical analyzer (DMA) capable of partitioning springback into elastic and anelastic (time independent and dependent) mechanisms.
\end{abstract}

For FY 2003, the main objective was to evaluate the hypothesis that plastic deformation alters the instantaneous elastic modulus $\left(\mathrm{M}_{\mathrm{E}}\right)$ of $\mathrm{Al}$ alloys. Earlier work using tensile deformation indicated a modulus reduction, but the plastic strain range that could be studied was limited and the reductions were small

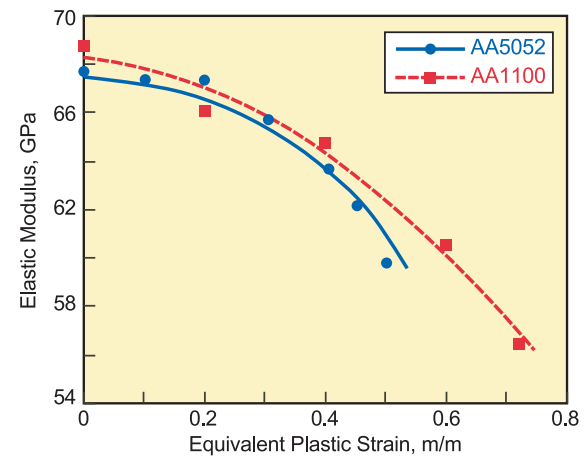

Figure 1: The measured elastic modulus of two aluminum alloys as a function of equivalent strain. compared to those produced by texture changes $(<<19 \%)$. Through DMA experiments on Al sheet deformed in biaxial tension, $\mathrm{M}_{\mathrm{E}}$ was shown to be lower by $12 \%$ to $18 \%$ by deformation. Although changing texture can lead to large changes in properties, with $\mathrm{M}_{\mathrm{E}}$ varying as much as $19 \%$ with direction in $\mathrm{Al}$ single crystals, texture measurements at the NIST Center for Neutron Research (Figure 2) combined with elastic moduli estimated for the measured orientation distribution functions (Figure 3) demonstrate unequivocally that plastic deformation lowers $\left(\mathrm{M}_{\mathrm{E}}\right)$ beyond that which can be produced by texture changes. These results were presented at quarterly meetings of the USCAR Springback Predictability consortia.

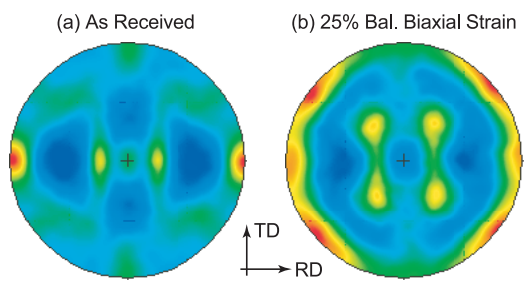

Figure 2: Measured (200) pole figures for AA5052 before and after balanced biaxial deformation.

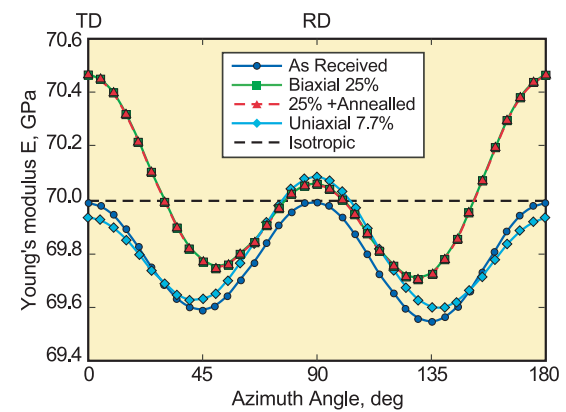

Figure 3: Predicted elastic moduli for measured pole figures and Al single crystal elastic constants.

Issues to be addressed in the future include prediction methods for elastic and anelastic modulii, development of standard practices for developing data, and a better understanding of underlying causes of the effect. While Figure 1 indicates that a simple empirical model can be used to incorporate these effects in springback FEA codes, the fundamental physics underlying this effect and its dependence on composition is not yet known.

\section{Contributors and Collaborators}

S.W. Banovic, D. Dayan, L. Ma, A. Munitz, D.J. Pitchure (Metallurgy Division); H. Prask, V. Luzin (NIST Center for Neutron Research); E. Chu (Alcoa); USCAR Springback Consortia (Alcoa, DaimlerChrysler, Ford, GM, LSTC, U.S. Steel) 


\section{Microstructural Origins of Surface Roughening and Strain Localizations}

Existing data, measurement methods, and the basic understanding of metallurgical factors that influence friction, tearing, and surface finish during sheet metal fabrication are insufficient to meet the finite element modeling requirements of industry. This project approaches the resultant need by exploring the microstructural origins of the distribution of slip, surface roughening, and strain localization during plastic straining. Influence of initial material characteristics on the formability of Al-and Fe-based sheet metals as a function of biaxial deformation is the primary focus of the investigations.

\section{Stephen W. Banovic and Mark R. Stoudt}

$\mathrm{R}$ eplacement of conventional steel sheets by aluminum alloys and high-strength low-alloy steels for various automobile closures is an effective means of reducing vehicle weight in an effort to increase fuel efficiency. However, widespread application of these materials by the automotive industry is limited primarily due to formability issues and material database/constitutive law deficiencies encountered in finite element modeling. Therefore, this project examines the underlying structure-property relationships associated with formability in an attempt to further the numerical simulations that predict deformation behavior of materials.

The main focus of this past year was to enhance the methodology of surface roughness measurements and to develop new analysis techniques to extract characteristics in the roughness data. By utilizing the appropriate analysis techniques, surface roughness measurements can be a tool to identify which deformation mechanisms were active, assess the roughening that is characteristic of that mechanism, and provide information regarding the range of activity of that mechanism as a function of metallurgical condition. Preliminary results from an ongoing study of the influence of strain path on surface roughness have recently shown that the resolution of the instrument is crucial to obtaining statistically significant results and understanding their meaning.

Figure 1 shows the topography obtained with scanning laser confocal microscopy (SLCM) of a $1 \mathrm{xxx}$-series aluminum sheet deformed in balanced biaxial tension. With traditional contact profilometry, the basic assessment of the roughness can be obtained, however the data do not distinguish between the baseline roughness and that which is characteristic of the plastic

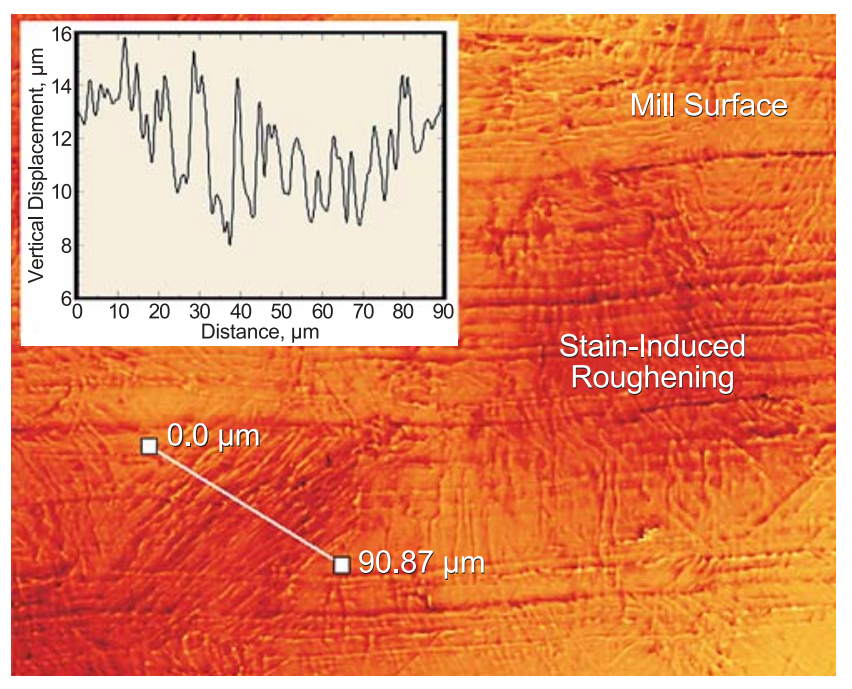

Figure 1: Surface topography of a 1xxx-series aluminum alloy, deformed to a true strain of 0.1, obtained using scanning laser confocal microscopy.

deformation. This is an inherent limitation caused by the profilometer stylus acting as a low-pass filter that cuts off the short wavelength component of the roughness and limits resolution of small surface features. In addition, specific microstructural features cannot be targeted by this method. Conversely, analysis with SLCM yields a broader spectrum of information about the roughness character. An example can be found in the inset of the figure, which displays the corresponding roughness profile from the $90 \mu \mathrm{m}$ line profile shown. The analysis provides considerable information about the height and distribution of the individual slip steps regardless of the surrounding features of the milled surface. With this additional information, a more accurate model of the roughening process in this material can be developed. These results are of significant interest to automotive manufacturers who have requested that we develop a NIST Recommended Practice Guide for Roughness Measurements.

Future work will focus on surface roughness development and crystallographic texture evolution of Al- and Fe-based materials deformed via multi-pass strain paths to more accurately simulate actual stamping operations.

\section{Contributors and Collaborators}

T. Foecke, R.E. Ricker, D. Dayan (Metallurgy Division, NIST); M.D. Vaudin (Ceramics Division, NIST), T. Gnäupel-Herold, V. Luzin (NIST Center for Neutron Research); Y. Shim (University of Georgia) 


\section{Plasticity, Fabrication Processes, and Performance}

Prediction of large strain plasticity under complex load histories, such as those applied during industrially relevant processes, is limited due to our limited understanding of the role played by microstructure. Advances in theoretical and modeling capabilities that include microstructure, guided by experimental measurements and validation, are needed to shed new light on this problem. For example, Object Oriented Finite Element (OOF) programs are limited by their inability to deal with plasticity. Remedying this is one of the principal challenges of this project.

\section{Richard Fields}

$\mathrm{D}$ ffraction provides a powerful means of very accurately measuring both microstructure and mechanical behavior in a way that provides insight simultaneously into both, and offers particular opportunities for understanding plasticity. This project is implementing one or more plasticity models in OOF and devising critical experimental and computational benchmarks to validate OOF using the NCNR's neutron diffractometer to measure residual stress, texture, and elastic properties. Conventional x-ray sources are also being used where stress states measured at or near surfaces are important for understanding the behavior of materials or to compare with predictions. Synchrotron x-rays are used to deal with subsurface residual stresses in the thin, highly textured samples that result from simulated forming operations. Specifically, the following tasks are underway:

- Experimentally validate calculations of the important role played by residual stresses on high-precision machining and forming operations (includes collaborative contributions from MEL, ALCOA, and Boeing Corporation);

- Provide technical assistance and validation testing to CTCMS staff engaged in adding 3D plasticity to OOF;

- Develop the ability to characterize near-surface residual stresses; and

- Further develop neutron and synchrotron radiation sources to provide high-resolution data needed to relate microstructure, residual stresses, and their effect on springback, etc.

Once plasticity has been implemented in OOF, additional verification tests will be carried out against analytical and numerical models. Validation tests will be carried out against benchmark experiments run at the NCNR and in the Metallurgy Division. An effort to include basic 3-D aspects to OOF based on stereology may be included in this part of the research.

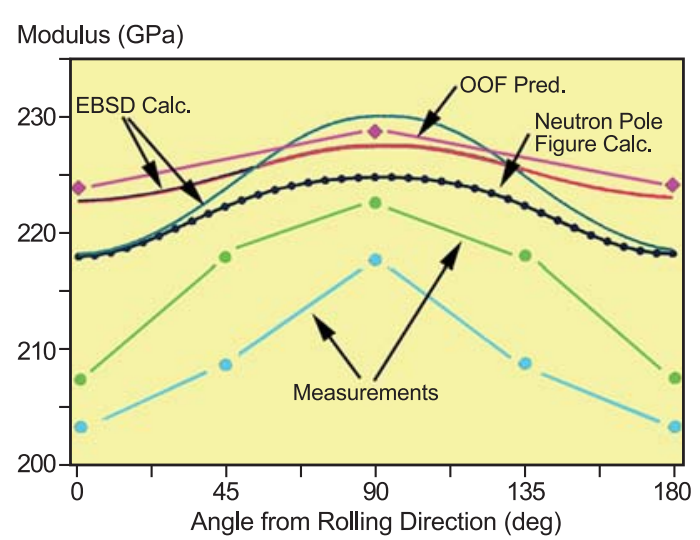

Figure 1: Comparison of measured elastic modulus dependence on texture with predictions using EBSD and OOF model.

\section{Accomplishments}

- Validation of Distortion Prediction Method: In 2002, we validated residual stress measurement and prediction methods of industry. In 2003, we generated shapes with measured distortion machined from self-stressed bodies to compare with industry-predicted behavior.

- Test of OOF: Textured polycrystalline samples were tested for elastic modulus dependence on texture. The results were compared with predictions using EBSD (Electron Backscatter Diffraction) and OOF (Figure 1). The predictions, while promising, require further study to understand differences between measurements, calculations, and predictions.

- Residual stresses in standard forming cups were measured and provided to the Forming Program project on Formability Standards. In addition, analyses were done to understand the origin of these stresses.

This project has targeted a need expressed by numerous companies to extend OOF calculations into the plastic range. It is also responsive to the metal forming research needs of the auto industry, as well as Boeing, ALCOA, and steel manufacturers with whom NIST currently collaborates. Improvements in predicting large plastic strain behavior under complex loading conditions will have significant benefits on product differentiation, time-to-market, and the cost of forming dies for U.S. products.

\section{Contributors and Collaborators}

L. Levine, R. deWit (Metallurgy Division, NIST); H. Prask, T. Gnäupel-Herold, V. Luzin (NIST Center for Neutron Research); R. Polvani (Manufacturing Metrology Division, NIST); D. Bowden (Boeing); E. Chu (ALCOA); R. Reno (University of Maryland) 


\section{Standard Tests and Data for Sheet Metal Formability}

In order to meet the USCAR/Freedom Car goals for fuel efficiency, the U.S. automotive industry is moving to lighter, high-strength materials for auto bodies. A NIST survey of industry has found that providing designers with accurate material properties, and a way to incorporate them into finite element models of sheet metal forming dies, is a critical need for the U.S. auto industry. This project seeks to develop new standard tests and metrology to accurately determine sheet metal mechanical response under forming conditions.

\section{Tim Foecke}

Tn order for the U.S. automotive industry to be able to transition to new materials for formed sheet metal parts, they must be able to mechanically characterize the starting materials under forming conditions, and input this information into die design models. In response to this need, we are developing sheet metal formability tests, along with associated metrology, that can be standardized and used by industry.

Springback is the elastic shape change to a part associated with the residual stresses that develop during the stamping process. This shape change complicates assembly and accurate fit-up, and the automotive industry wants to either avoid it or at least be able to predict its magnitude and design dies to account for it. The proposed test for springback consists of splitting open a ring cut from the sidewall of a deep drawn cup.

In 2003, we have concentrated on establishing the robustness of the springback cup test to experimental errors, in preparation for submission of the test to ASTM, and measurement of residual stresses within the cups using x-rays and neutrons. Errors in ring location and geometry, as well as blank misplacement, produced changes in the springback opening. It was found that displacing the ring location vertically along the sidewall of the cup produced the greatest effect (10\% per $\mathrm{mm}$ of error), and that blank misalignment greatly increased data scatter. The former is related to the effect of changing thickness on the ring's area moment of inertia and the latter on thickness variations around the ring. Standardization is planned via a round-robin analysis coordinated through ASTM.

New this year is the installation of an x-ray stress measuring system on the sheet metal formability station (Figure 1) that will lead to a standard test for measuring multiaxial flow surfaces. This new instrumentation allows for direct, in situ measurement

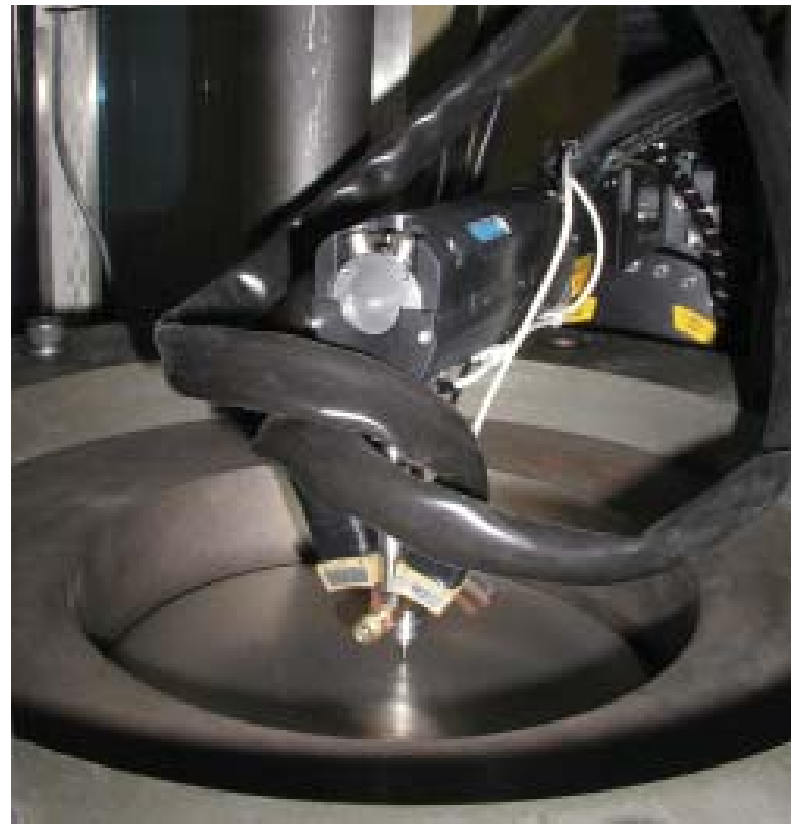

Figure 1: Equipment for measuring stress simultaneously with forming operation.

of the stress in a given direction while the sample is under biaxial load. Using a series of deformation steps and the sample geometries previously developed to produce multiaxial strain states, one can, for the first time, directly measure the multiaxial flow surface of a sheet metal. The facility is able to test both steel and aluminum samples but can be upgraded to test any metal.

Three other tasks using these new experimental capabilities have been initiated. The first is a collaboration with GM and ALCOA on developing stress-based forming limits, where the multiaxial stress-strain surfaces measured will be used to verify path independence of this formalism. The second involves studying the percentage of retained austenite transformation in TRIP automotive sheet steel as a function of strain state and level, in collaboration with U.S. Steel. Finally, stress-based hole expansion forming limits will be examined by looking at the stress-strain response of the material immediately adjacent to a hole expanding in multiaxial tension. This last project is in collaboration with GM.

\section{Contributors and Collaborators}

T. Gnäupel-Herold (NIST Center for Neutron Research); M. Shi (USS); E. Chu (ALCOA); C. Xia (Ford); T. Stoughton, M. Wenner, C.T. Wang (GM); A. Andersson (Volvo); E. Schedin (Avestapolarit) 


\section{Underlying Processes of Plastic Deformation in Metal Alloys}

A substantial increase in the use of aluminum alloys and high-strength steels in automobiles would greatly increase fuel efficiency. The primary reason why this has not yet occurred is a lack of accurate deformation models for use in designing the stamping dies. This project is developing a physically based model of plastic deformation using a combination of statistical physics approaches and advanced measurement techniques.

\section{Lyle E. Levine}

$\mathrm{P}$ lastic deformation of metals (as in cold rolling, stamping, drawing, and metal fatigue) is a topic of great importance to industries worldwide, and improvements in the basic technology would have a significant effect on our economy. The design of new metal products is often accomplished by computer simulation of the production process using empirically derived constitutive equations. Unfortunately, existing constitutive equations cannot accurately predict the material behavior, and many tryout and redesign steps are required. Another related difficulty is in the design of new alloys with improved formability characteristics. Currently, alloy design is done empirically with little understanding of how the various constituents affect the mechanical properties.

To address both of these issues, this project is focused on developing constitutive laws based upon the underlying physical processes that produce the observed mechanical behaviors in metal alloys. Such constitutive laws are inherently multi-scale since they must describe phenomena on length scales ranging from the atomistic all the way to the macroscopic stress-strain behavior of bulk material.

Since the underlying processes are extremely complex, we have concentrated almost all of our theory, modeling, and experimental efforts over the past several years on single-crystal pure $\mathrm{Al}$ as a model system. This work is reaching completion with the development of the segment length distribution (SLD) model, which describes how macroscopic deformation arises from the statistical behavior of large numbers of dislocations.

The project was in a state of transition this year. With work on the SLD model nearing completion, the focus is shifting into several new directions. The first is a multi-institutional effort to obtain experimental measurements to validate and provide inputs into the new NIST models. Our partners include researchers at
Washington State University who are funded by DOE for four years to conduct chemi- and photo-emission studies of Al alloys deformed in situ. These experiments will measure the time-intensity behavior of individual slip events that are predicted by NIST researchers. The dislocation substructures of the samples will be studied by researchers at Los Alamos National Laboratory. NIST researchers are making the theoretical predictions and designing an ultra-high-vacuum tensile stage. NIST will also grow all of the single-crystal alloy samples.

A second new direction is incorporation of atomistic-level processes into the SLD model, allowing chemical effects to be addressed. A collaboration with NIST's Chemical Science and Technology Laboratory is beginning, and a jointly funded postdoc joined us in August 2003. This work should allow quantitative predictions to be made of the effects of different alloying elements on mechanical behavior during plastic deformation.

The third new direction in progress is a preliminary look at whether the SLD model can be extended to $b c c$ metals. This task involves growing shaped, single-crystal samples of a $b c c \mathrm{MgLi}$ alloy and deforming them in tension. The resulting dislocation structures will be studied using TEM, and the surface slip structures will be imaged using a variety of microscopic techniques.

Finally, we are working with researchers at Oregon State University to answer long-standing questions about the existence and magnitude of longrange residual stresses that occur during deformation. $\mathrm{X}$-ray diffraction techniques are often used to measure these stresses, but interpretation of the results is subject to question. We are addressing this lack through both synchrotron X-ray experiments and computer simulations.

NIST researchers also co-organized two large international symposia including one on multiscale modeling at the MRS spring meeting in San Francisco (April 21-25) and one on dislocations at Plasticity 2003 in Quebec City, July 7-11.

\section{Contributors and Collaborators}

D. Pitchure, M.R. Stoudt, R.M. Thomson (Metallurgy Division, NIST); K. Luedek (SURF student); A. Chaka (Physical and Chemical Properties Division, NIST); D. Shechtman (University of Maryland); T. Dickinson, S. Langford, M. Cai (Washington State University); M. Delos-Reyes, M. Kassner (Oregon State University); R. McCabe (LANL); G. Levi (Technion, ISRAEL) 


\section{Atomization Processing - Recommended Practices}

Powder Metallurgy (PM) is used to produce a large number of metallic materials that benefit from the enhanced homogeneity and reduced machining afforded by this process. Higher performance PM products and process economics impose increasing demands on the control of powder size during the gas atomization process. The NIST Metallurgy Division atomization facility has been a test-bed for providing the PM industry with material property data and processing procedures for improving yield of desirable powder size distributions. This information is being compiled into a comprehensive "Gas Atomization Recommended Practice Guide."

\section{Stephen D. Ridder and Steven Mates}

$\mathrm{G}$ as Atomization is the primary source of material for many advanced Powder Metal (PM) processing procedures such as Powder Injection Molding (PIM), Hot Isostatic Press (HIP) consolidation, and Thermal Spray (TS) coatings. These processes are used to produce surgical tools and appliances, aircraft and power turbine engine parts, severe service parts for the petrochemical industry, and increasing numbers of critical automotive components. Powder size, because of its affects on microstructure, powder handling, and consolidation, controls the resulting PM material properties.

The cost to produce these optimum powder size distributions can become prohibitively expensive when using traditional gas atomization techniques that require screening of the powder product for usable powder and recycling the waste. Research collaborators representing the major gas atomization companies and national laboratories have participated in various projects aimed at sensor development, alloy chemistry, particle size control, and powder characterization. Current contents in a NIST Special Publication, "Gas Atomization Recommended Practice Guide," include:

- Sensors and control techniques to optimize the Gas-to-Metal mass flow-rate Ratio (GMR);

- Gas jet geometry designs that reduce adverse shock wave generation; and

- Advanced process control software that incorporates atomization plume video frames and "knowledge base" heuristics.

Additional research topics of interest to the PM industry will be published in supplements to the NIST Special Publication. A new topic under investigation is the effect of "swirl," a tangential velocity imposed on the atomization gas flow, on atomizing behavior.

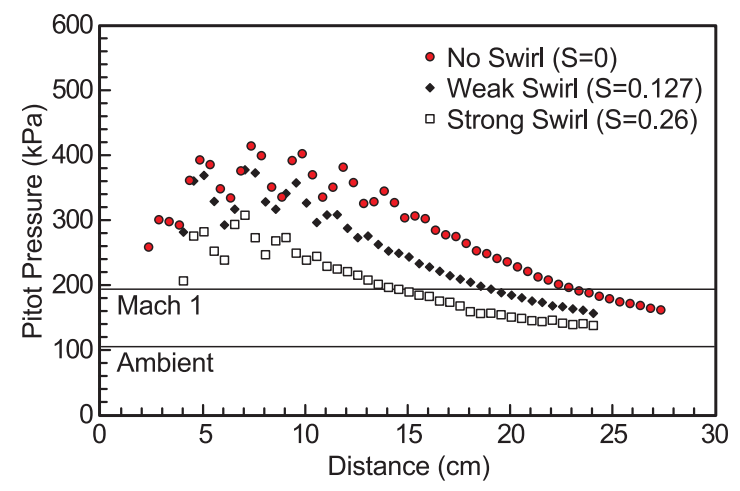

Figure 1: Effect of swirl strength on axial gas velocity distribution produced by an annular atomizer.

Swirl is sometimes imparted to the gas flow in an attempt to enhance melt breakup in the near region. However, swirl is known to speed turbulent diffusion, a process that leads to rapid attenuation of the gas velocity downstream of the near region. As a result, secondary atomization is potentially hindered, resulting in wider particle size distributions or unwanted coarse particles. We are currently quantifying the effect of swirl strength on the aerodynamic behavior (Figure 1) and atomization performance of generic nozzles meant to simulate current industrial practice.

In addition, a small-scale gas atomizer has been assembled to atomize small heats (less than $500 \mathrm{~g}$ ) for the purposes of studying both bulk metallic glass formation and particle size/processing parameter relationships.

The ultimate goal of the latter is to deduce more accurate particle size prediction equations that can be used to boost efficiency in industrial powder production operations.

Ongoing collaboration with industrial partners continues to be an important component of liquid metal atomization research at NIST and technology transfer of the results of our previous atomization research.

"The research history of NIST, and background of the scientists involved with our project at NIST, give the company confidence that we will reach a best-possible solution within the bounds which are dictated by the real, practical limitations of our plant."

John Askeland

Operations Manager, Ampal Corp.

\section{Contributors and Collaborators}

F.S. Biancaniello, P.A. Boyer, R.D. Jiggetts, D. Wickner (Metallurgy Division, NIST); John Askeland (AMPAL) 


\section{High Speed Machining}

The U.S. spends $\$ 200$ billion annually on machining of metal parts. The rapid development of high-speed machines and tooling makes traditional knowledge-based approaches to improved efficiency ineffective: a recent survey found that industry chooses the correct machining tool less than half of the time. Modeling has been promoted by industry as a method to realize improved efficiency. Through this project, NIST will develop measurement capabilities and deliver materials data for use in machining simulation software.

\section{Richard J. Fields and Carelyn E. Campbell}

$\mathrm{M}$ achining of metal parts to required dimensional tolerances is an essential component of the manufacturing process. Efficient processes can greatly reduce the price of parts and enhance the competitive position of the manufacturer. Industry now requires that traditional, experience-based approaches to finding optimal process parameters, such as feed rate, cutting rate, tool material, tool shape, etc., be replaced by modeling and simulation. Commercial simulation codes exist to assist this optimization. However, the codes require a realistic materials property database and validation. NIST will publish data for use in these codes by: (1) measurement of mechanical property data at high strain and heating rates; and (2) characterization and modeling of the deformation modes and microstructure changes that occur during machining. Residual stresses, responsible for part distortion after machining, are being measured at the Center for Neutron Research. Thermal imaging of machining is being performed by the Optical Technology Division.

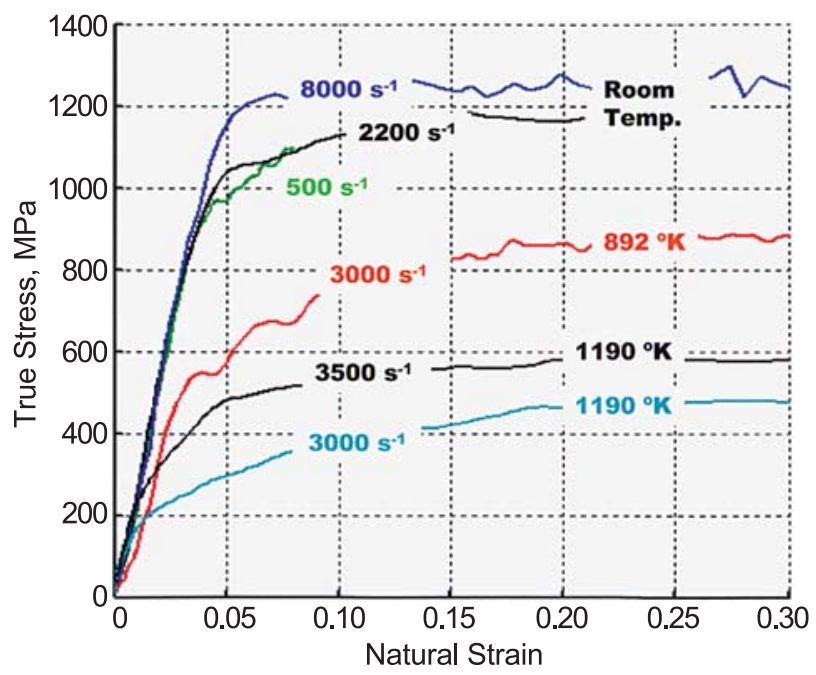

Figure 1: Stress/strain behavior of AISI 1045 steel tested at various temperatures and high strain rates.
The first task has developed a unique testing facility for measuring mechanical behavior of alloys under conditions close to those occurring in machining operations; i.e., at strain rates up to $10^{4} \mathrm{~s}^{-1}$ with concurrent heating to test temperatures at rates up to $5 \times 10^{5} \mathrm{~K} / \mathrm{s}$. Complete stress $/$ strain data can be recorded with better than microsecond resolution. The temperature is known with similar temporal resolution. This facility has been used this year to develop a database and test various constitutive laws for AISI 1045 steel, an important alloy for machining. In Figure 1, the behavior of this steel at various high strain rates and temperatures is shown. Samples of 7075-T61 aluminum alloy are also being prepared for mechanical testing with this instrument.
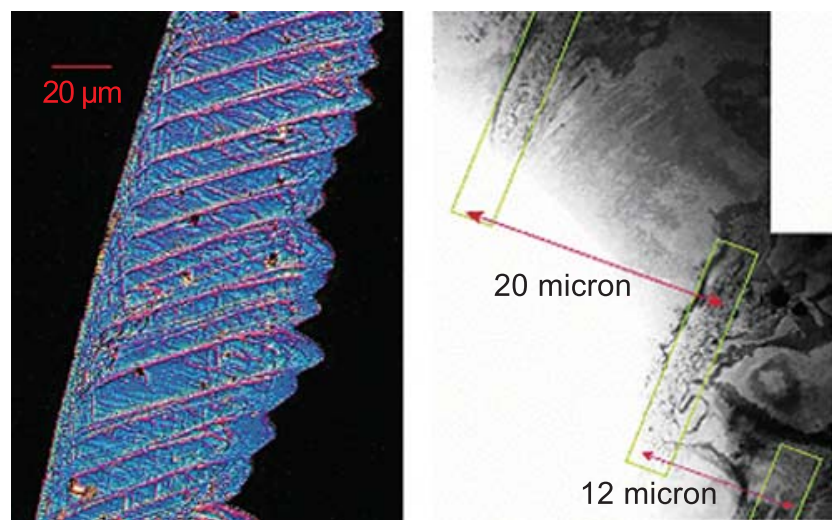

Figure 2: Optical (left) and TEM (right) images of 7075 chips with localized deformation and recrystallization.

The second task focuses on microstructure changes during machining. Initial work examined severely heated and deformed chip material. Transmission electron microscopy reveals that only slight coarsening of the precipitate structure occurs, but significant changes in grain size are evident. Figure 2 shows that shear bands seen in optical micrographs contain dynamically recrystallized grains. Similar characterization is being performed on the work piece. A microstructural model based on the microstructural characterization and the expected temperature and strain rate histories during machining is being developed.

\section{Contributors and Collaborators}

D. Basak, W.J. Boettinger, L. Bendersky, L.E. Levine (Metallurgy Division, NIST); R. Rhorer, R. Polvani, K. Jurrens, M. Kennedy, R. Ivester, and H. Soons (Manufacturing Metrology Division, NIST); B. Dutterer (Fabrication Technology Division, NIST); T. Burns (Mathematical and Computational Sciences Division, NIST); H. Yoon (Optical Technology Division, NIST); M. Davies (North Carolina State University) 


\section{Phase Field Modeling of the Electrochemical Interface}

\author{
Phase field modeling techniques developed in \\ the last decade have added considerably to the \\ understanding of solidification microstructures \\ such as dendrites, grains, and eutectics. NIST \\ Metallurgists have taken, and continue to take, \\ a leading role in these developments. This project \\ seeks to extend NIST's "phase field toolkit" to \\ encompass electrochemical processes.
}

\section{Jonathan E. Guyer}

\begin{abstract}
$\mathrm{A}^{\mathrm{s}}$ s manufacturing industries increase their reliance on microstructure-dependent properties, control over microstructure becomes critical to product performance. Process modeling has become an important input to design of many manufacturing processes. As an example, the microelectronics industry uses electrochemistry to form the intricate array of interconnects in advanced microprocessors. Traditional electrochemical models make a number of assumptions that break down at these length scales, particularly when voids can form or disappear.
\end{abstract}

Many of the same morphological features are found in both solidification and in electrodeposition. The phase field technique has had considerable success in modeling these features during solidification. (For a brief description of the method and initial application to electrochemical interfaces, see Technical Highlights.) We are able to model both the equilibrium and kinetic behavior seen in electrochemical experiments and in earlier models while making a minimal set of assumptions. Despite these successes, the electrochemical problem is a particularly challenging application of the phase field method, and there are a number of barriers to overcome before we can model macroscopic 2D systems.

A trivial phase field model of a solid-liquid interface can be constructed with just one variable: the phase field itself. To apply the method to an electrochemical interface, we must also consider the electrostatic potential and a minimum of three independent charged species: electrons, cations, and anions. Instead of one governing equation, the minimal electrochemical model is thus governed by five, each of which is coupled to all of the others. The simplest electrochemical phase field model is intrinsically more difficult to solve than even some fairly complicated solidification phase field models.

Quantitative results are obtained from a mathematical model by rendering the equations on a discrete mesh of points or elements. Typical phase field models require five to ten mesh points to adequately resolve the interface between phases. Because of the short range of electrostatic

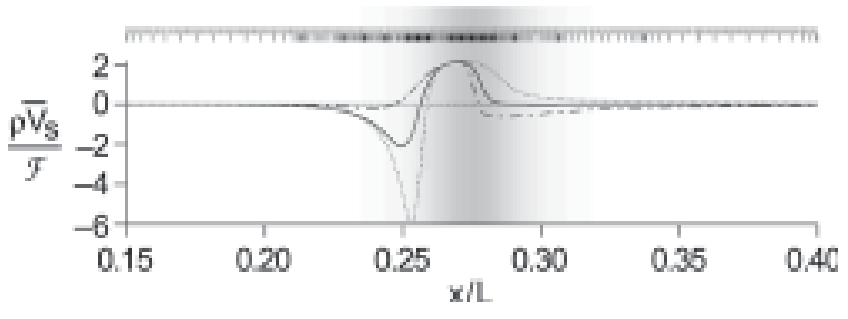

Figure 1: Calculated charge distribution in the electrochemical "double layer" for different interface potentials. The tick marks above illustrate the uniform and adaptive computational meshes required to obtain accurate numeric solutions. The gray band shows the location of the diffuse phase field interface.

interactions, we need an order of magnitude more mesh points to resolve the charge distribution in the electrochemical interface, as illustrated in Figure 1.

An adaptive meshing technique was also applied. In this approach, mesh points are concentrated at the interface as needed, resulting in considerably faster computations from a good initial guess (calculations take a few minutes instead of a few days). Unfortunately, the algorithm does not converge at all from a poor guess, whereas the simpler uniform mesh code can eventually obtain a solution from almost any initial condition. The two approaches were applied in concert to obtain the results described in our earlier Technical Highlight.

In the algorithm we have applied so far, chosen for its simplicity of implementation, computation time is proportional to the square of the number of points. Considering that our present $1 \mathrm{D}$ calculations of a $1 \mathrm{~nm}$ section through the interface can take upwards of three days on a fast computer, a naïve approach to $2 \mathrm{D}$ calculations of the same domain could take 10,000 years. Macroscopic features such as dendrites would take many orders of magnitude longer to model. Faster computers can mitigate some of this computation time, but practical solutions will require more efficient numerical algorithms.

In the next year, we will focus on more sophisticated algorithms that minimize the cost of adding mesh points. In parallel, we will reduce the total number of mesh points through adaptive meshing techniques. Although the particular adaptive technique used in our 1D solutions cannot be scaled to $2 \mathrm{D}$, the general approach should significantly reduce computation times.

\section{Contributors and Collaborators}

W. Boettinger, J. Warren (Metallurgy Division, NIST); G. McFadden (Mathematical and Computational Sciences Division, NIST) 


\section{Processing of Amorphous Metals}

Recently developed metal alloy chemistries that solidify amorphously in cross sections approaching several centimeters are the focus of increasing interest in the production of large, meter-scale components by powder consolidation for use in a variety of challenging military, aerospace, and industrial applications. The Metallurgy Division is combining rapid solidification processing and instrumented powder consolidation to study the production and densification of several classes of these new amorphous alloys.

\section{Frank Biancaniello and Rodney Jiggetts}

$\mathrm{R}^{\mathrm{c}}$ ecently developed bulk amorphous metal (BAM) alloys have been shown to exhibit plastic behavior in the absence of dislocations, resulting in materials with high-yield strength, high modulus, and high hardness. These include low density aluminum-based amorphous materials identified by Boeing that may replace titanium in jet engines and high density hafnium-based alloys being developed by the Army.

The Army's interest in high-density BAM alloys is a result of their need for a new armor piercing projectile material to replace the current Depleted Uranium (DU) technology. Although DU is a very effective projectile material, the resulting radioactive and otherwise toxic debris is difficult to remove from the environment. The desirable DU properties include the high mass density $\left(19070 \mathrm{~kg} / \mathrm{m}^{3}\right)$ and the adiabatic shear localization (ASL) that occurs when rapidly loaded. High density provides a benefit in energy and momentum transfer, and ASL leads to a "self sharpening" of the projectile as it penetrates armor plate rather than the less desirable plastic deformation and "mushrooming" of the projectile tip (thus degrading penetration depth). The Army has tested several alternative materials, and, to date, the best have been composites of tungsten powder within metallic matrices. The $19,300 \mathrm{~kg} / \mathrm{m}^{3}$ density of $\mathrm{W}$ is close to DU and the ideal metallic matrix will have properties such that the resulting composite fails with ASL or ASL-like behavior. Recent work has shown that BAM alloys have promise in providing the necessary ASL-like behavior.

The need for large-size BAM components $(>10 \mathrm{~cm}$ cross section) and suitable materials for the projectile composites has resulted in an interest to produce BAM alloy particles for subsequent consolidation. Atomization of a pre-alloyed melt will produce fully amorphous solidification in powder particles $<100 \mu \mathrm{m}$ in diameter. In addition, thermodynamic assessments of candidate alloys will be used to study a range of amorphous materials with the necessary mechanical properties. Figure 1 shows the small-scale gas atomizer that was built to study the composition-dependent solidification behavior of BAM alloys using small batch $(250 \mathrm{~g})$ processing to reduce material costs. Large-scale batch processing of candidate compositions for consolidation studies is then accomplished using the NIST $22 \mathrm{~kg}$ melt capacity, gas-metal atomizer.

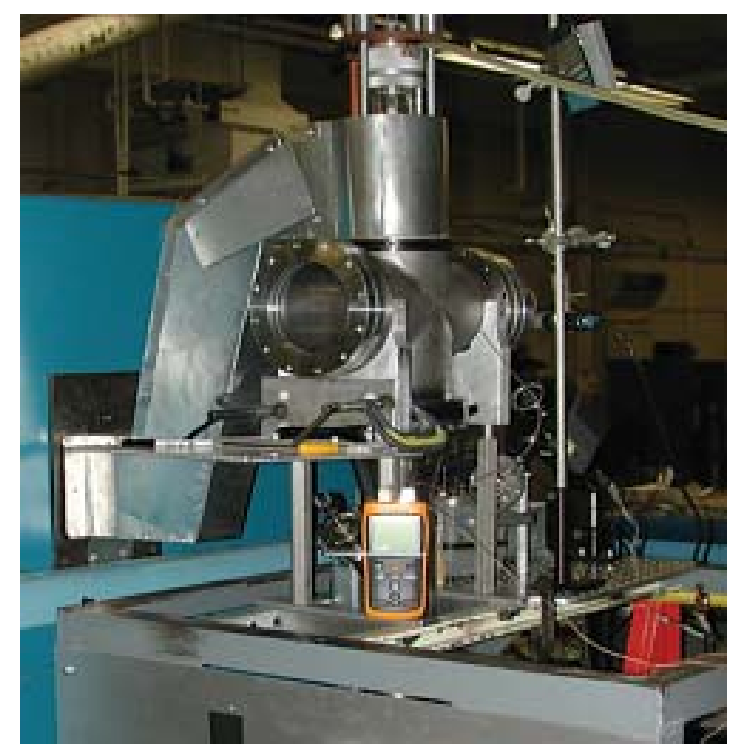

Figure 1: Small scale Laval atomizer for production of $250 \mathrm{~g}$ lots of metal powder.

In order to take full advantage of the benefits afforded by BAM alloys, powders must be consolidated without inducing large-scale re-crystallization. To understand the densification behavior of these materials, a project was initiated to study the kinetics of consolidation by pressure and heat below the crystallization temperature. A hot isostatic press (HIP) has been instrumented with a sensor developed at NIST to follow densification. A warm isostatic press that uses a liquid ester working fluid that can be pressurized to $400 \mathrm{MPa}$ (twice the pressure of the HIP) is also being instrumented with a density sensor. These two units provide quantitative, real-time monitoring of the progress of densification.

\section{Contributors and Collaborators}

R.J. Fields, S.P. Mates, T. Pierce, S.D. Ridder (Metallurgy Division, NIST); T. Zahrah, R. Rowland (MatSys); D. Bowden (Boeing); L. Christodoulou (DARPA); L. Kecskes (U.S. Army) 


\section{Combinatorial Methods}

The Combinatorial Methods Program develops novel high-throughput measurement techniques and combinatorial experimental strategies specifically geared towards materials research. These tools enable the industrial and research communities to rapidly acquire and analyze physical and chemical data, thereby accelerating the pace of materials discovery and knowledge generation. By providing measurement infrastructure, standards, and protocols, and by expanding existing capabilities relevant to combinatorial approaches, the Combinatorial Methods Program lowers barriers to the widespread industrial implementation of this new R\&D paradigm.

The Combinatorial Methods Program has adopted a two-pronged strategy for accelerating the development and implementation of combinatorial approaches: an active intramural $R \& D$ program and an ambitious outreach activity. The intramural R\&D program is designed to better tailor combinatorial methods for the materials sciences and extend the state-of-the-art in combinatorial techniques. Measurement tools and techniques are developed to prepare and characterize combinatorial materials libraries, often by utilizing miniaturization, parallel experimentation, and a high degree of automation. A key concern in this effort is the validation of these new approaches with respect to traditional "one at a time" experimental strategies. Accordingly, demonstrations of the applicability of combinatorial methods to materials research problems provide the scientific credibility needed to engender wider acceptance of these techniques in the industrial and academic sectors. The successful adoption of the combinatorial approach also requires a highly developed "workflow" scheme. All aspects of combinatorial research, from sample "library" design and library preparation to high-throughput assay and analysis, must be integrated through an informatics framework, which enables iterative refinement of measurements and experimental aims. Combinatorial Methods Program research projects give illustrations of how combinatorial methodology is implemented effectively in a research setting.

NIST-wide research collaborations, facilitated by the Combinatorial Methods Program, have produced a wide range of new proficiencies in combinatorial techniques, which are detailed in a brochure, "Combinatorial Methods at NIST" (NISTIR 6730), and online at www.nist.gov/combi. Within the Materials Science and Engineering Laboratory (MSEL), novel methods for combinatorial library preparation are designed to encompass variations of diverse physical and chemical properties, such as composition, film thickness, processing temperature, surface energy, chemical functionality, UV-exposure, and topographic patterning of organic and inorganic materials ranging from polymers to nanocomposites to ceramics to metals. New instrumentation and techniques have been developed that enable the high-throughput measurements of adhesion, mechanical properties, failure mechanisms, film thicknesses, and refractive index, among others. The combinatorial effort extends to multiphase, electronic, optoelectronic, and magnetic materials, including biomaterials assays. On-line data analysis tools, process control methodology, and data archival methods are also being developed as part of the Program's informatics effort.

The extensive outreach activity in the Combinatorial Methods Program is designed to facilitate technology transfer with institutions interested in acquiring combinatorial capabilities. The centerpiece of this effort is the NIST Combinatorial Methods Center (NCMC), an industry-university-government consortium organized by MSEL that became operational on January 23, 2002 via a kick-off meeting in San Diego. The recognized importance of NCMC activities is readily apparent, as 15 industrial partners, the Air Force Research Lab, and the University of Southern Mississippi are participating in the NCMC membership program. The membership continues to grow and $80 \%$ of the members from last fiscal year have already renewed for this fiscal year. The NCMC facilitates direct interaction between NIST staff and these industrial partners, and it provides a conduit by which Combinatorial Methods Program research products, best practices and protocols, instrument schematics and specifications, and other combinatorial-related information can be effectively disseminated. This outreach is mediated in large part by a series of semi-annual workshops for NCMC members. Indeed, since its inception, four NCMC workshops have been held at NIST. Further information on the NCMC can be found on the website at www.nist.gov/combi.

Contact: Leonid A. Bendersky 


\section{Combinatorial Methods for Thin Films}

In the search for new functional materials for use in modern microelectronics, various thin film oxides are under consideration. With the ability to locally measure properties of the films, combinatorial approaches using miniature thin film assays of varying compositions can be of significant impact to optimize the search. The combinatorial methodology is still in its early stages of development, and, therefore, understanding the fundamentals of synthesis of the combinatorial assays, as well as the relationships between crystal chemistry and physical properties of the assays, is of great importance.

\section{Leonid A. Bendersky}

$\mathrm{T}_{\mathrm{t}}^{\mathrm{h}}$ The effectiveness of the combinatorial approach in the development of thin film electronic materials has been clearly demonstrated. In order to rapidly survey a large compositional landscape, assays with continuously changing compositions are synthesized, processed, and screened in a single experiment. Dielectric, magnetic, and other properties can be measured within compositional spreads using recently developed instruments with high spatial resolution, such as the microwave microscope and microSQUID. In early combinatorial materials research, the local microstructure and phase composition were largely unprobed. Through our ongoing collaboration with Professor Ichiro Takeuchi from the University of Maryland, we have demonstrated the value of using transmission electron microscopy (TEM) for understanding the deposition process and the correlations between physical properties and local microstructure.

In 2003, our primary focus was on using combinatorial research to demonstrate tunable bandgaps by doping a semiconductor $\mathrm{ZnO}$ (bandgap: $3.3 \mathrm{eV}$, hexagonal structure) with $\mathrm{MgO}$ (bandgap: $7.8 \mathrm{eV}$, cubic structure). Potential applications are for optical devices, including UV lasers, as well as transparent conducting layers for solar cells and phosphors. The $\mathrm{Zn}_{1-\mathrm{x}} \mathrm{Mg}_{\mathrm{x}} \mathrm{O}$ thin film assays are prepared using pulsed laser deposition (PLD) with synchronized shutters. Multiple targets were alternatively ablated for atomic layer-by-layer deposition onto substrates with matching lattices, producing epitaxial films compositionally uniform in the growth direction and with one-dimensional compositional variation along the film plane. These epitaxial spreads were studied for bandgap by UV transmission and for microstructure by scanning $\mathrm{x}$-ray microdiffractometer and transmission electron microscopy (TEM).
The results show a continuously changing bandgap for $\mathrm{x}<0.4$ and $\mathrm{x}>0.6$, suggesting possibilities of using the arrays themselves as photodetectors with a range of detection frequencies separately active at different locations within the film.

Scanning x-ray results showed three structurally different regions. TEM gave insight into the structural details. An especially important finding was for the $0<\mathrm{x}<0.4$ region where, according to the $\mathrm{x}$-ray diffraction, there is an extended solid solution of $\mathrm{Mg}$ in $\mathrm{ZnO}$. TEM, however, identified a high density of planar defects and, according to high-resolution imaging, the defects appear to be layers of (111) $\mathrm{MgO}$ intergrown in the host lattice of $\mathrm{ZnO}$ (see Figure 1). Thus the effect of $\mathrm{Mg}$ ions on the electronic structure and bandgap of $\mathrm{ZnO}$ will be very different, at least conceptually, from the solid-solution model.

Other active areas of research include developing MOIF as a rapid imaging method to determine ferromagnetic phase boundaries, establishing the relationship between bulk properties and thin film properties in shape memory alloys, and measuring composition-dependent and structure-dependent properties of multiferroic materials.

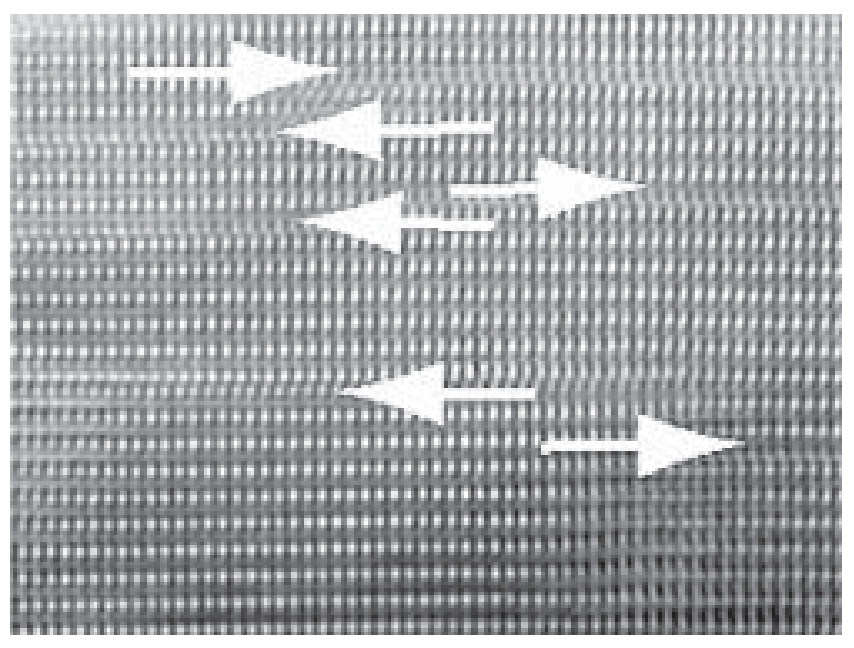

Figure 1: Epitaxial ZnO-based phase (containing 10 at \% Mg) with a high density of extended planar defects. HRTEM image showing the stacking fault-type nature of the defects and suggesting an accommodation of the excess $\mathrm{MgO}$ on the defect.

\section{Contributors and Collaborators}

I. Takeuchi, K.-S. Chang (University of Maryland); M.J. Turchinskaya, R. Parke (Metallurgy Division, NIST) 


\section{Phase Diagrams for III-V Thin Films and Metal Contacts}

Commercialization of Group III nitride devices has been hindered by several technological and fundamental materials issues, including problems with making high quality GaN epilayers and bulk substrates and with fabrication of reliable electrical contacts. This project focuses on experimental and computational evaluation of Metal - Group III - N phase diagrams to be used as a roadmap for: a) optimization of growth and thermal processing of GaN and related nitrides and for; b) development of metallization information to enable the design of improved electrical contacts to GaN-based devices. For metallizations, a combinatorial approach is used for establishing the relationship between electrical properties, metallization compositions, and processing parameters.

\section{Albert Davydov and William Boettinger}

G allium nitride-based semiconductors are used in blue-green and UV lasers, LEDs, photodetectors, and high-temperature/high-power electronic devices. The optimum growth conditions of GaN, choice of contact metals, and thermal processing limits for the device structures rely mostly on trial-and-error methods and are not fully understood. We believe that knowledge of the thermodynamics/kinetics in Metal Group III - N systems can provide guidance for optimization of the device processing parameters.

In the Ga-N system, we use experimental and computational CALPHAD procedures to evaluate the thermodynamic and phase diagram data (Figure 1). A thermodynamically consistent model representation of the available data and our experimentally determined properties have resulted in reasonable estimates of

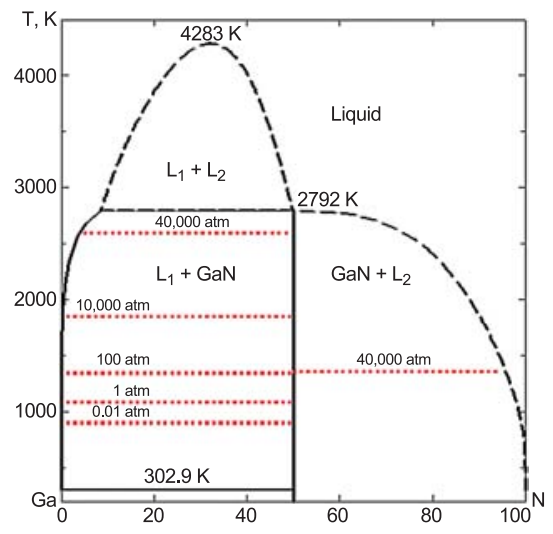

Figure 1: Calculated Ga-N phase diagram with superimposed isobaric lines (dashed).

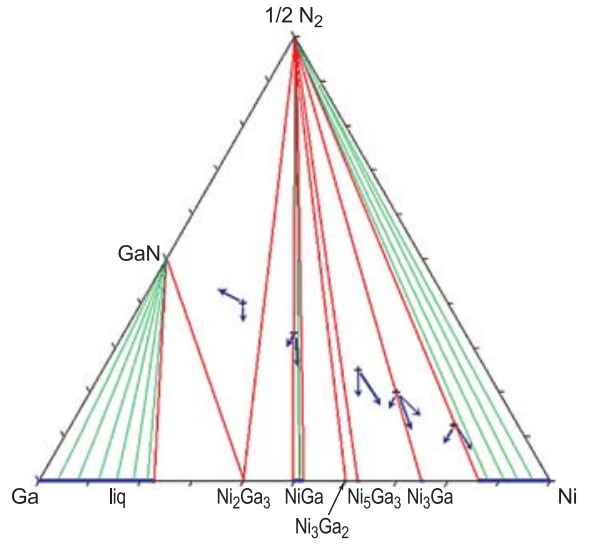

Figure 2: Experimental data (points and arrows) and calculated (lines) for the $\mathrm{Ni}-\mathrm{Ga}-\mathrm{N}$ phase diagram.

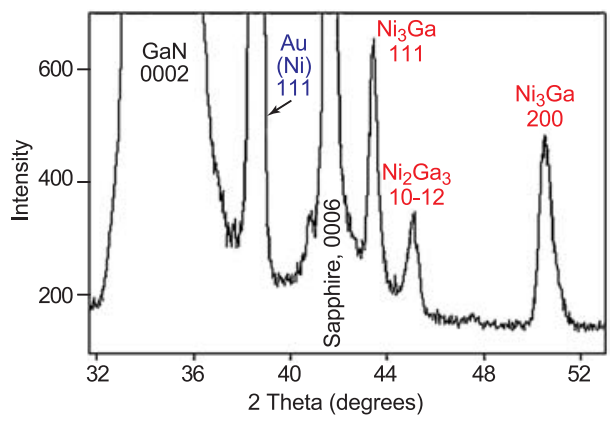

Figure 3: XRD of $\mathrm{Ni} / \mathrm{GaN}$ thin film structure (overcoated with $\mathrm{Au}$ ) annealed at $800^{\circ} \mathrm{C}: \mathrm{Ni}_{3} \mathrm{Ga}$ and $\mathrm{Ni} \mathrm{G}_{2} \mathrm{Ga}$ are formed.

missing critical data, such as melting/decomposition temperature of $\mathrm{GaN}$ as a function of nitrogen pressure. Results are now posted on the ONR National Compound Semiconductor Roadmap website (http://ncsr.csci-va.com) and can be used in optimizing growth and processing conditions for $\mathrm{GaN}$-based devices.

In 2003, the utility of phase diagrams in analyzing metallization choices was demonstrated using $\mathrm{Ni}$ as one of the most commonly used industrial contacts. The Ni-Ga-N phase diagram was calculated and verified experimentally (Figure 2). The diagram was used to interpret the interfacial reactions in the $\mathrm{Ni} / \mathrm{GaN}$ thin film structures (see Figure 3). An experimental combinatorial study of $\mathrm{Ni} / \mathrm{Au}$ metallization to $\mathrm{GaN}$ is now underway.

\section{Contributors and Collaborators}

L. Bendersky, U. Kattner, F. Biancaniello, D. Josell, A.J. Shapiro (Metallurgy Division, NIST); T.J. Anderson, O. Kryliouk (Univ. of Florida); S. Noor Mohammad (Howard University); M. Master (TDI, Inc.) 


\section{Data and Data Delivery}

The economic importance of technical data has grown steadily over the past three decades. NBS/NIST took an important lead early in the effort to emphasize the pervasive use and increase the impact of reliable materials property data. As early as 1985 , former NBS Director E. Ambler noted that the need for property data had become a "national priority," while the National Research Council repeatedly observed that there is a persistent "critical national need" for materials property data.

The NIST Materials Science and Engineering Laboratory (MSEL) has been a prominent leader in responding to this national need. By design, the scope of the MSEL effort is evolutionary and responds to the ever-increasing advances in technology. Currently, MSEL has six project areas in the Data and Data Delivery program.

\section{- Crystallographic and Phase Equilibria Databases} [Project Leader - V. Karen]: This project encompasses the two most venerable efforts in MSEL, each of which recently has had a major new release. In collaboration with Fachinformationszentrum Karlsruhe (FIZ, Germany), a CDROM version of the FIZ/NIST Inorganic Crystal Structure Database (ICSD) has been released providing the full structural data, i.e., lattice parameters and atomic coordinates, for approximately 60,000 compounds. The long-standing collaboration between NIST and the American Ceramic Society (ACerS) has continued with the completion of a new topical volume in the NIST/ACerS Phase Equilibria Diagrams series, the first of two planned volumes on electronic ceramics.

\section{- Phase Equilibria and Properties of Dielectric} Ceramics [Project Leader - T. Vanderah]:

An integrated theoretical and experimental effort is underway to predict and measure phase equilibria and electronic behavior in dielectric oxide systems. This work includes relaxor ferroelectrics, dielectrics for cellular infrastructure and hand-held devices, and dielectrics for low temperature co-fired ceramics for applications in multilayer ceramic integrated circuit technology. The impact of this work was evident in Dr. Vanderah's invited perspective article, "Talking Ceramics," which appeared in the journal Science. The timeliness of this work was emphasized further by its subsequent feature in the science section of the New York Times.

\section{- Phase Relationships in High Temperature} Superconductors [Project Leader - W. Wong- Ng]: MSEL's meticulous effort to provide phase information critical to the development of practical superconductors is currently directed towards two important systems: $\mathrm{Ba}_{2} \mathrm{RCu}_{3} \mathrm{O}_{7-\mathrm{x}}$, where $\mathrm{R}$ is yttrium or a lanthanide, and $\mathrm{MgB}_{2}$. For $\mathrm{Ba}_{2} \mathrm{RCu}_{3} \mathrm{O}_{7-\mathrm{x}}$, work has focused on the phase relations in $\mathrm{BaF}_{2}-\mathrm{BaO}-\mathrm{Y}_{2} \mathrm{O}_{3}-\mathrm{CuO}_{\mathrm{x}}-\mathrm{H}_{2} \mathrm{O}$ and the interactions of $\mathrm{Ba}_{2} \mathrm{RCu}_{3} \mathrm{O}_{6+x}$ with buffer layers, both of which are important for advances in the " $\mathrm{BaF}_{2}$ ex situ" process and the "liquid-phase-epitaxy" process. For $\mathrm{MgB}_{2}$, the enthalpy of formation, vapor pressure, and sources of variability have been determined.

- Reaction Path Analysis in Multicomponent Systems [Project Leader - C. Campbell]: Many industrial processes rely on multicomponent diffusion to control the formation and dissolution of precipitate phases. MSEL is developing a multicomponent diffusion mobility database for $\mathrm{Ni}$-base superalloys that will be used, for example, to predict the formation of the $\gamma^{\prime}$ (ordered FCC) phase during the solidification of superalloys. A workshop, "High Throughput Analysis of Multicomponent Multiphase Diffusion Data," was held in March 2003 to focus on the development of methods to extract diffusion data from multicomponent diffusion couples.

- Evaluated Materials Property Data [Project Leader - R. Munro]: Engineering designs utilizing advanced materials require reliable data. Elasticity, strength, toughness, hardness, creep, thermal expansion, conductivity, diffusivity, and durability are prominent among the data categories needed and desired for materials applications and development. This project is directed both towards the development of evaluated databases of these properties for structural and superconducting ceramics and towards the establishment of the evaluation methodologies that form the foundation of reliable materials property data systems. A significant achievement in this effort is "Data Evaluation Theory and Practice for Materials Properties," SP960-11, the eleventh NIST Recommended Practice Guide produced by MSEL.

- Informatics and Visualization in Materials Data Delivery [Project Leader - C. Beauchamp]: The internet, and the World Wide Web in particular, has become a dominant resource medium for technical information. MSEL has undertaken a major commitment to make its extensive data collections available via this medium. New efforts, now underway, will provide web access to the MSEL lead-free solder materials property database and the diffusion data archive that is important for the processing of metal alloys. Additionally, the internet will be used increasingly as a means of disseminating MSEL's prodigious technical publications in the form of electronic manuscripts.

Contact: Carlos R. Beauchamp 


\section{Informatics and Visualization in Materials Data Delivery}

Success in implementing a reliable materials design is dependent on access to accurate and reliable properties data. With the shortened cycle between product concept and commercial rollout and the increased permutations of materials choices, being able to deliver critically evaluated data in a timely manner has become one of the prime objectives of the Metallurgy Division. Efforts are concentrated on using the World Wide Web to deliver, in standard format such as MatML (a standard materials mark-up language), the data gathered across all projects.

\section{Carlos Beauchamp and Carol Handwerker}

$\mathrm{T}$ he effectiveness of searching electronic databases is presently hampered by the lack of materials data that is properly formatted to enable such searches. To address those needs, the Metallurgy Division has embarked on two major projects to make collections of critically evaluated data available on the Internet.

The first of these projects addresses the collection of over 25,000 international papers, theses, and government reports on diffusion published before 1980 and warehoused in the NIST Diffusion Data Center in printed form. The recent NIST-sponsored workshops on "Databases for Computational Thermodynamic and Diffusion Modeling," (March 2002) and on "High Throughput Analysis of Multicomponent Multiphase Diffusion Data," (March 2003) highlighted the need for access to the diffusion data stored at the Center to be able to proceed with the development of multicomponent diffusion databases, which are based on binary and ternary diffusion data already contained there and with the modeling of diffusion-controlled processes.

Work is underway to convert the bibliographical data contained in the collection into electronically searchable data that will be available through a designated link in the Division homepage.

The second of these efforts addresses the need for a better understanding of the mechanical properties of lead-free solder alloys and their thermo-mechanical responses (e.g., Figure 1). This knowledge is required for the implementation of accelerated test plans, predictive reliability models, and product reliability assessment.

The service life of solder joints is limited by the fatigue damage accumulated in the material. The reliability analysis used to predict the service life of solder joints under use conditions, based on accelerated tests, depends on life prediction models based on $\mathrm{SnPb}$ assemblies. Similar models are non-existent for the lead-free solders due to the lack of understanding of failure modes, adequate constitutive models, and reliability databases that correlate failure times under test with real field conditions.

The Division is committed to providing a quantitative review of the thermo-mechanical properties of lead-free solders, emphasizing the $\mathrm{SnAgCu}$ system, the choice of alloy for reflow assemblies, and the SnAg system, recommended for wave-soldering applications. The results of the report are in the process of being converted into electronically available data, along with the complete report, through the Division's homepage.

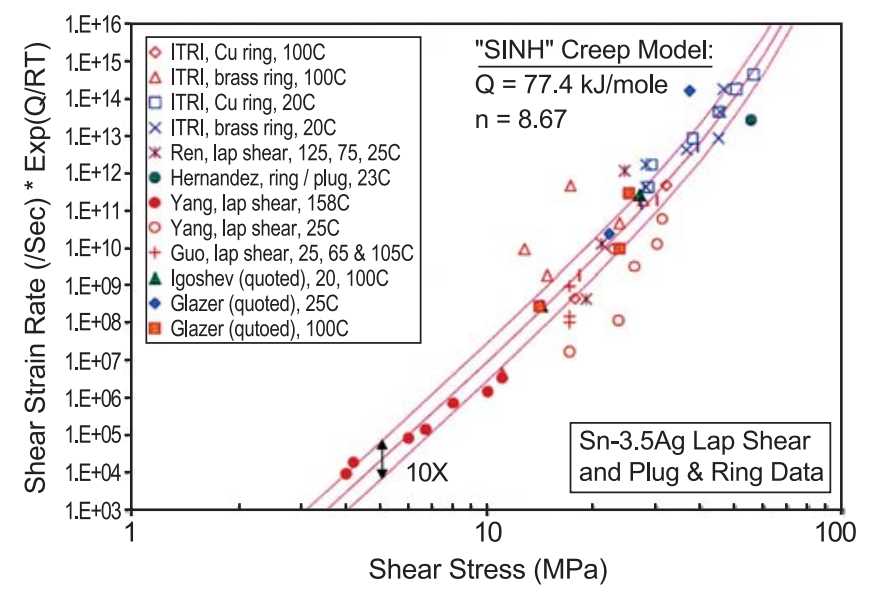

Figure 1: Sn-3.5Ag lap shear and plug \& ring data.

Additional efforts in MSEL include:

- Developing approaches for viewing multidimensional mechanical property and phase diagram data for metals;

- Continued support for the development and implementation of MatML, a materials mark-up language;

- Compiling elastic moduli data for polycrystalline oxide ceramics;

- Completing the Inorganic Crystal Structure Database (in cooperation with Fachinformationszentrum, FIZ, Germany); and

- Producing phase diagrams through NIST/American Ceramic Society Phase Equilibria Program.

\section{Contributors and Collaborators}

W.J. Boettinger, C.E. Campbell, K. Dotterer, R. Drew, R. Fields, H. Gates, F. Gayle, U.R. Kattner, K.-W. Moon, S. Tobery (Metallurgy Division, NIST); J.-C. Zhao (GE); J.P. Clech (EPSI, Inc.) 


\section{Reaction Path Analysis in Multicomponent Systems}

Many commercial processes rely on multicomponent diffusion to control the formation and dissolution of precipitate phases within the matrix phase or at interfaces. This project develops diffusion mobility databases that provide efficient methods of storing a wealth of diffusion data and enable extrapolation of the available data to higher-order systems.

\section{Carelyn E. Campbell and William J. Boettinger}

$\mathrm{M}$ ulticomponent diffusion controls many industrial processes, especially in the processing and application of Ni-based superalloys. An improved understanding of diffusion-controlled microstructures would aid optimization of heat treatments that avoid incipient melting and would enable the prediction of freezing temperatures and solidification paths for systems such as Ni-based superalloys. These processes require mobility databases to describe the composition-dependent diffusion coefficients.

The NIST composition-dependent mobility database has been constructed using an approach that assumes that quaternary and higher-order interactions are negligible. Thus, binary and ternary effects can be combined to extrapolate to higher-order systems. For application to nickel-based superalloys, we have constructed a database for the $\gamma$ (disordered FCC) phase of the Ni-Al-Cr-Co-Fe-Hf-Mo-Nb-Re-Ta-Ti-W system. The database was published in Acta Materialia in 2002 where it is readily available to industry. General Electric Corporate Research and Development (GE-CRD) and Howmet Corporation provided experimental results from multicomponent diffusion couples which were compared with diffusion simulations. The experimental and calculated diffusion composition profiles for the Rene88/IN718 diffusion couple agree quite well, as shown in Figure 1. The current database is now being refined based on experimental work from GE-CRD.

Two applications of this work are mentioned here. As a part of a GE-led DARPA/Advanced Insertion of Materials project, NIST supplies composition dependent diffusivity data for the $\gamma$ matrix that team member Questek Innovations uses in numerical modeling of the $\gamma^{\prime}$ precipitation process. NIST has also initiated an effort to develop heat treatment optimization techniques to address industry's need for more efficient heat-treating practices of cast Ni-based superalloys. The ideal heat treatment of a cast Ni-base superalloy consists of a heating schedule that avoids incipient melting while minimizing either the power expenditure or the heating time to achieve a homogeneous single-phase

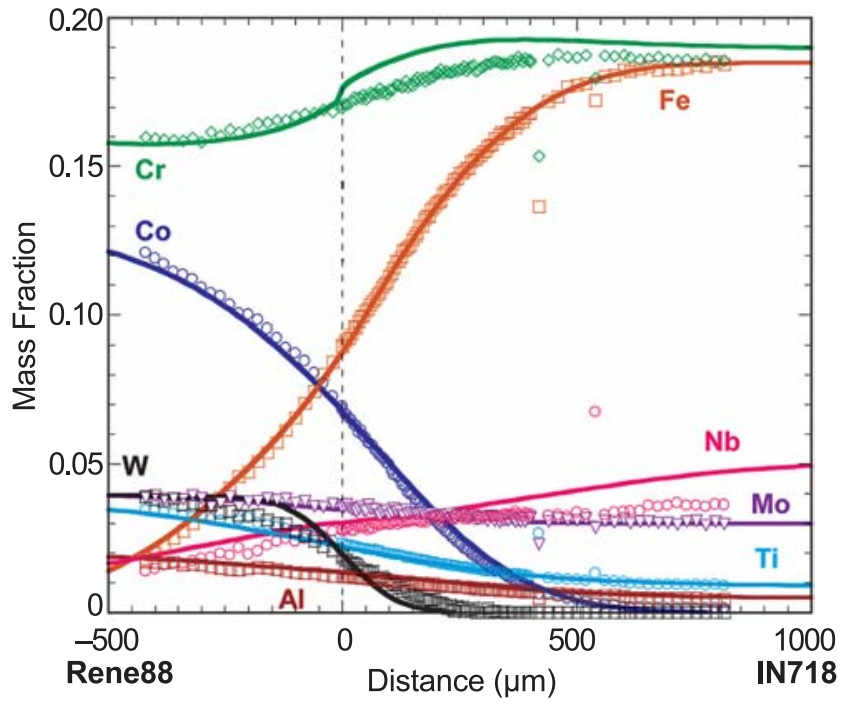

Figure 1: Calculated (solid lines) and experimental (symbols) composition profiles for the Rene88/IN718 diffusion couple at $1150{ }^{\circ} \mathrm{C}$ for $1000 \mathrm{~h}$. (Experimental data from $G E-C R D$.)

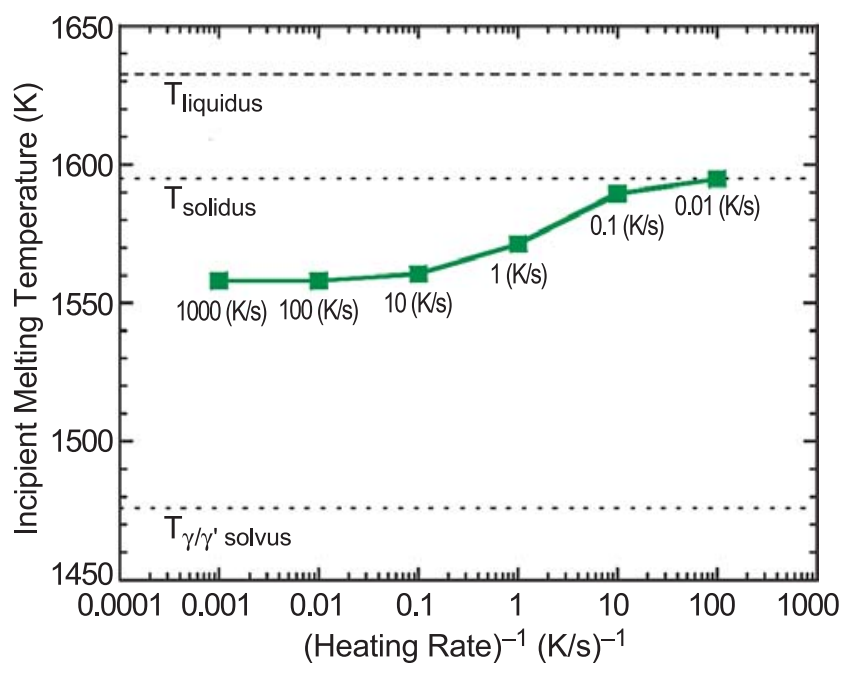

Figure 2: For Rene-N4, the predicted incipient melting temperature $(K)$ as function of linear heating rates.

structure. For the solidified Rene-N4 composition profile calculated using a constant cooling rate of $1 \mathrm{~K} / \mathrm{s}$, Figure 2 shows the predicted incipient melting temperature as a function of linear heating rates.

\section{Contributors and Collaborators}

U. Kattner (Metallurgy Division, NIST); J.-C. Zhao (GE-CRD); T. Hansen (Howmet); C. Kuehmann (Questek Innovations); Z.K. Liu (Penn State); D. Seidman (Northwestern University) 


\section{Nanocharacterization}

The emphasis on nanotechnology around the world and the successful implementation of the National Nanotechnology Initiative in the U.S. are accelerating the development of science and technology at the nanoscale. Nanotechnology is expected to play a key role within the next 10 years in a wide spectrum of industry sectors including manufacturing, information technology, electronics, and healthcare. Novel devices at the micro- and nanoscale will become increasingly important in all of these industries. The ability to measure dimensions, characterize materials, and elucidate structures of new and novel materials at the nanoscale will be critical to the advancement of nanotechnology. In addition, one of the exciting prospects of nanotechnology lies in the ability of molecules or particles, under specific conditions, to self-assemble to form new materials with unusual properties. Successful development of these new materials will require the ability to monitor such processes at the nanoscale in real time. Metrology, the science of measurement, is therefore the foundation of nanotechnology. Standards and reference materials will also provide essential infrastructural support to this emerging technology.

The objective of the program in the NIST Materials Science and Engineering Laboratory (MSEL) is to develop basic measurement metrology at the nanoscale for the determination of bulk and surface material properties and for process monitoring. Measurement methods are being developed for use in conjunction with new instrumentation and calibration artifacts.

The scope of the program encompasses metals, ceramics, and polymers in various forms - particles, thin films, nanotubes, and self-assembled structures — and also includes studies of nanocomposites and liquid-state properties for microfluidics-based fabrication and measurement techniques. Physical properties such as mechanical strength, elastic moduli, friction, stiction, adhesion, and fatigue strength are measured, as well as the size of nanoparticles and the structure and dispersion behavior of nanoparticulate systems. Other properties such as electrical conductivity, thermal conductivity, magnetic properties, electronic properties, and optical properties are also examined. While the program focuses on developing measurement techniques at the nanoscale, proper data interpretation requires fundamental studies in nanomechanics, scaling laws, and imaging techniques.

There are currently ten projects under the program:

- Bridging Length Scales in Theory and Modeling;

- Electrochemical Processing of Nanoscale Materials;

- Metrology for Nanoscale Properties;

- Nanofiller Processing;

- Nanoindentation;

- Nanomechanics and Standards;

- Nanoscale Manufacturing;

- Nanotribology and Surface Properties;

- Particle Metrology and Standards; and

- Physical Properties of Thin Films and Nanostructures.

In many of these individual projects, objectives are directed toward the study of a particular class of materials or material properties, but the underlying theme of the program as a whole is to develop reliable, accurate measurement techniques for a broad range of materials and material properties at the nanoscale. As just one example, four methods for the determination of the elastic properties of thin films atomic force acoustic microscopy, surface acoustic wave spectroscopy, Brillouin light scattering, and nanoindentation - are being compared using common sets of specimens. This study will lead to a better understanding of the complementary nature of these techniques for measuring nanostructured materials and their combined use to determine supplementary properties such as film thickness or density. Standard reference materials are being developed in collaboration with other National Measurement Institutes around the world for use in the verification of the performance of these instruments.

Contact: James A. Warren 


\section{Bridging Length Scales in Theory and Modeling: Microstructure Evolution and Material Mechanics}

The performance of many structural and functional materials depends on the distribution of composition, phases, grain orientations, internal stresses, and microstructure on length scales from nanometers to meters. Industry needs predictive models to reduce the enormous costs and development times involved in designing and inserting new materials into state-of-the art products. To address these needs, we are developing phase field models of solidification and solid-state transformations, with coupling to stress, grain orientation and solute/vacancy dynamics.

\section{James A. Warren and William J. Boettinger}

$\mathrm{I}$

$\mathrm{n}$ order to model the complex interfacial shapes that produce real microstructures, the phase field method has become the technique of choice for computational materials scientists.

Modeling of microstructures produced by processing of materials involves solution of mathematical equations for heat flow, deformation/fluid flow, and/or solute diffusion. Boundary conditions on external surfaces reflect the macroscopic processing conditions that can be externally controlled. In classical models of materials processing, boundary conditions on internal interfaces corresponding to the liquid-crystal (grain), grain-grain and/or phase-phase interfaces reflect material thermodynamic and kinetic conditions. For solid-solid transformations, stress effects also become significant, and surface stresses become particularly important in nanoscale microstructures. Phase field models finesse the difficult numerical task of tracking moving internal interfaces by the introduction of a new variable, the phase field.

Research in phase field modeling conducted in the Metallurgy Division is also supported by the NIST Center for Theoretical and Computational Materials Science. Recent efforts have focused on bringing together many of the disparate efforts in phase field modeling to create a unified picture of materials processing with a single set of equations governing microstructure evolution. In the past year we have:

- Developed a model of a stressed system containing a curved interface between two phases with different elastic properties. This model has been applied to a liquid (zero shear modulus)-solid system.

- Simulated 3-D fault dynamics in directionally solidified binary eutectics.
- Combined phase field models of eutectic colony growth with polycrystalline structures. This approach readily deals with complex interface shapes and topology changes (see Figure 1).

- Simulated the growth of dendrites in polymers where crystallographic rotation of the growth direction occurs, "dizzy dendrites" (with the Polymers Division).

- Prepared an overview of phase field modeling of polycrystalline growth and coarsening (Acta Materialia, in press).

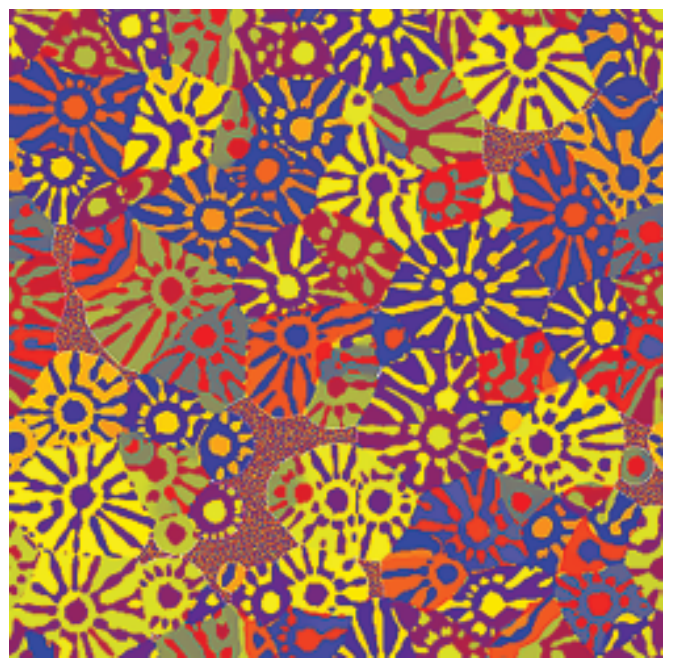

Figure 1: A simulation of eutectic colony growth. The colors indicate crystal orientation. The solid phase can be distinguished as large regions of a single color while the liquid appears essentially random in orientation at the smallest scales.

While this research is ambitious in its scale, the successes so far suggest that the goals are within reach. Work to be done includes joining the stress models with existing models of polycrystalline growth, the addition of vacancies to all of these models in a thermodynamically consistent manner, the extension of the grain boundary model to three dimensions, and the coupling of all of these effects to fluid flow.

\section{Contributors and Collaborators}

J. Warren, W. Boettinger, D. Lewis, J. Guyer, R.F Sekerka (Metallurgy Division, NIST); J. Douglas (Polymers Division, NIST); J. McFadden (Mathematical and Computational Sciences Division, NIST); J. Slutkser, A. Roytburd (University of Maryland); R. Kobayashi (Hokkaido University, Japan); L. Granasy (RISSPO, Hungary) 


\section{Electrochemical Processing of Nanoscale Materials}

Precise temporal control of supersaturation provided by electrochemical methods holds promise for the synthesis and characterization of materials and structures of very small dimensions - nanomaterials. Variation of the electrochemical potential leads not only to changes in the metal deposition rate but also effects the adsorption of surfactants and segregation phenomena which exert a strong influence on the morphological evolution and stability.

\section{Thomas P. Moffat and Jon Mallett}

$\mathrm{E}^{\mathrm{l}}$ ectrochemical synthesis has become an important tool in the fabrication of nanoscale devices and structures. Modulation of the electrode potential can be used to create films with a corresponding variation in composition and/or structure. This allows materials, such as multilayers and nanocontacts, to be engineered with submonolayer precision. Likewise, knowledge of the stability and reliability of these finite size structures will be central to any technical application.

In the last year, we have continued using scanning probe microscopy to characterize the structure and stability of immersed surfaces. This work ranges from atomically resolved STM studies of adsorbate dynamics to AFM measurements of roughness evolution during electrodeposition. The focus of this activity is strengthening our knowledge of the connection between atomistic processes and morphological evolution observed at larger length scales.

A new aspect of our program this year is the study of nanocontacts formed by electrodeposition between two electrodes. Physical property measurements of these junctions have revealed a variety of effects such as quantized conductance, ballastic magnetoresistance,

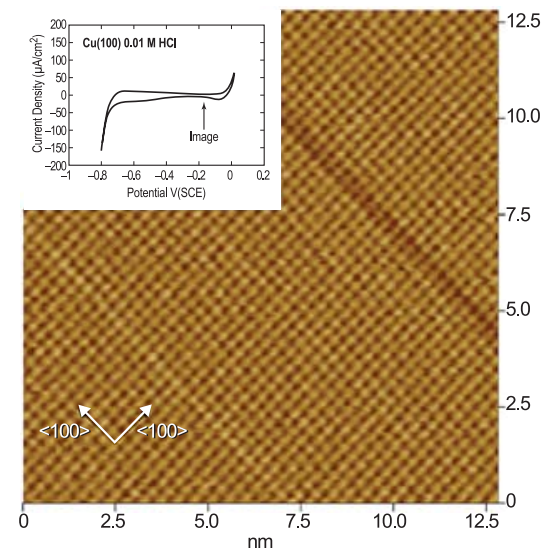

Figure 1: A high resolution STM image of a c(2x2) chloride adlayer on $\mathrm{Cu}(100)$ in $0.01 \mathrm{~mol} / \mathrm{L} \mathrm{HCl}$. The image was collected at $-0.150 \mathrm{VSCE}$ as shown on the inset voltammogram.

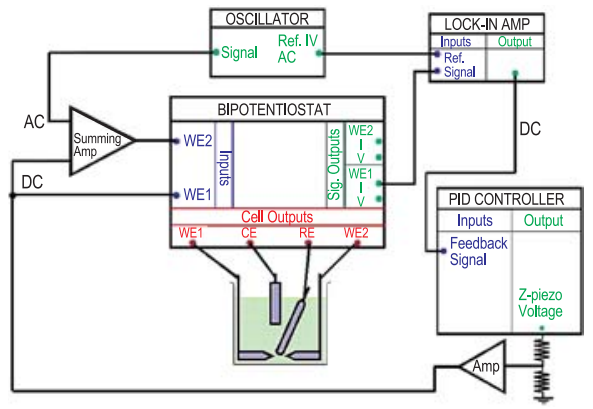

Figure 2: Schematic of control circuit used for forming and maintaining contacts of fixed conductance.

and adsorbate-influenced surface scattering. Many investigations use electrodeposited contacts, yet limited techniques are available for growing and maintaining such junctions at a fixed or predetermined conductance. At NIST, we have combined a bipotentiostat with a STM-like feedback circuit in order to form electrodeposited metal contacts of predetermined fixed resistance.

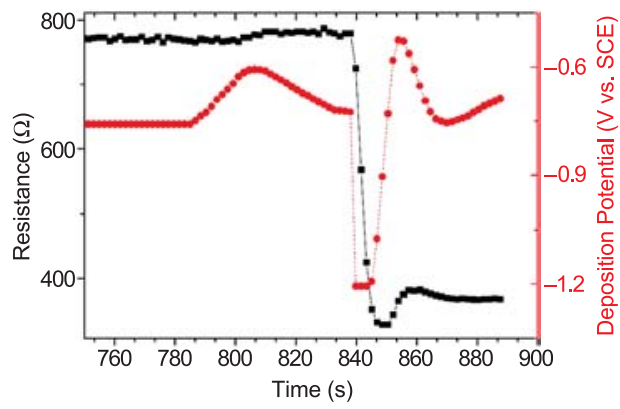

Figure 3: Controlled step in nanocontact resistance.

The junction conductance measured with a lock-in-amplifier is compared with a preset value, and the electrochemical potential of the two working electrodes is adjusted to either deposit or etch material to obtain the specified conductance value. Figure 3 shows a controlled transition in contact resistance between $778 \Omega$ and $369 \Omega$. Careful choice of the feedback control parameters resulted in transients with only one damped oscillation. Stable contacts have been grown between $800 \Omega$ and $2 \Omega$.

In the coming year, the measurements outlined above will be extended towards problems relevant to micro- and molecular electronics.

\section{Contributors and Collaborators}

W.F. Egelhoff, H. Ettedgui, E.B. Svedberg, J.E. Bonevich, R.D. McMichael (Metallurgy Division, NIST) 


\section{Interface of Materials with Biology}

New materials and devices are radically changing the medical treatment of injury and disease, yet because of the rapid pace of this segment of the materials industry, an adequate measurement infrastructure does not yet exist. The program on the Interface of Materials with Biology develops measurement methods, standards, and fundamental scientific understanding at the interface between materials science and biological science. Within the health care industry, we focus on dental and medical sectors that apply synthetic materials for replacement, restoration, and regeneration of damaged or diseased tissue. Three primary foci exist within this program: biocompatibility, materials properties, and materials science techniques applied to biological systems.

Whether the medical issue involves implanting a hip or knee joint prosthesis, a synthetic bone graft, or a tissue engineering scaffold into the human body, one of the primary issues is biocompatibility. By using our expertise in materials science, we are working to develop suitable Reference Materials (RM) for investigating biocompatibility and implant suitability. Research has focused on measuring the cellular response to powders and bulk materials to identify suitable candidates. We are collaborating with the American Dental Association Foundation (ADAF) to develop metrology methods to characterize the biocompatibility of synthetic bone grafts. Quantitative methods being developed include assays for adhesion, viability, proliferation, and differentiation of bone cells, as well as optical coherence tomography and confocal microscopy for measuring tissue ingrowth. We are developing biochemical assays to quantify inflammatory responses to synthetic materials. Finally, current research is working to bridge the gap between knowledge generation by cell biologists and product development in industry. In collaboration with the Chemical Science and Technology Laboratory, we are developing measurement methodologies and reference materials to use in assessing interactions in complex systems of living cells with synthetic materials. The expected outcomes of this work are reference substrates that induce specific cellular responses and engineered DNA vectors to act as fluorescent reporters of cellular responses.

In addition to the issue of biocompatibility, it is critical that the materials can withstand the mechanical and environmental stresses placed on them. For metallic implants, one concern is the corrosion pitting resistance of the implant materials and the associated potential for stress corrosion cracking (SCC). To address this issue, metal standards are being subjected to a simulated biological environment which will then be used to develop tests to assess the susceptibility for SCC.

Mechanical properties issues also arise when considering synthetic bone grafts and tissue engineering scaffolds. In addition to traditional bulk mechanical property measurements, combinatorial approaches are used to identify compositions and surface features that affect properties such as biocompatibility and mechanical durability. Finally, because the dental industry is primarily composed of small manufacturers with limited R\&D capability, collaborations with the ADAF, located in MSEL, are filling the gap by developing improved materials and techniques, patenting and licensing these inventions, and, most importantly, providing a technical foundation. Research focuses on improved understanding of the synergistic interaction of the phases of polymer-based composites and the mechanisms of adhesion to dentin and enamel. This approach will ultimately lead to materials with improved durability, toughness, and adhesion to contiguous tooth structure.

In this era of interdisciplinary approach to research, we provide an added dimension. By taking a physical/mechanical approach to how cells function, respond, and remodel in interaction with synthetic materials, we can provide skill sets typically absent in the biomedical community. Our concentration on mechanical property metrology extends to biological systems, spanning a considerable size range from individual neurons and muscle cells to complete pulmonary arteries. This necessitates the development of unique mechanical testing platforms and application of a materials science approach to understanding integrated properties.

Fundamental to much of the work in this program is the recognition that surfaces and interfaces play a critical role in biological systems and, in particular, in the interactions of synthetic or designed materials with biological systems and function. By applying the expertise in the NIST Materials Science and Engineering Laboratory to characterization of surfaces and interactions at interfaces in biomaterials, we will accelerate the introduction of improved materials and help provide the means to assure quality control that is critical to this industry.

Contact: Frank S. Biancaniello 


\section{Biocompatibility of Metals Reference Powders}

The medical implant device industry needs characterized metal particles to be used in evaluating the effect of debris released by metallic implants. There is also a need to determine what surface characteristics are needed in metallic implants to improve biocompatibility. This research is developing expertise directed at investigating the biocompatibility and implant suitability of Powder Metallurgy (PM) processed 316L stainless steel (SS), PM processed High Nitrogen SS, and traditional ingot processed $316 \mathrm{~L}$ SS.

\section{Frank Biancaniello and Stephen Ridder}

$\mathrm{R}$ oughly two billion dollars are spent each year in the United States on metallic medical implants. These include the large variety of hip and knee joint prostheses, dental implants, plates and pins for broken bones, vascular stents, and other reconstructive devices. Evaluation of the biomechanical performance of these devices involves in vitro and in vivo tests to access the suitability of a metal alloy chemistry or component design for its intended application. For example, artificial hip and knee joints are tested to determine strength, rate of joint wear, biocompatibility of wear debris, and nature of ingrowth at the implant/ bone interface. Comparisons of results from various laboratories are hindered by the lack of suitable reference materials to quantify biomechanical performance.

Gas atomized metal powders are an ideal source of particulate metallic materials for clinical testing. In addition, consolidated metal powder provides a means to generate reproducible reference metallic substrates with the enhanced chemical homogeneity and grain refinement found in PM processed materials. In the Metallurgical Processing Laboratory, we have the best instrumented gas atomization facility in the U.S., specifically designed to control particle size, solidification rate, and microstructure. In this small scale project, we are examining the utility of using well-controlled, gas-atomized powders produced at NIST as reference materials through an evaluation of metal powders and bulk metal specimens using several standard biocompatibility test methods. Genotoxicity will be determined using a bacterial reverse mutation assay (Ames Test), and acute cytotoxicity will be evaluated by the MTT cell proliferation assay using mouse fibroblasts.

During 2003, these biocompatibility experiments were run on three metallic powder and bulk materials:
1) ingot processed 316L (medical grade stainless steel for orthopaedic fixtures and screws); 2) PM processed 316L; and 3) PM processed High Nitrogen Stainless Steel (HNSS).

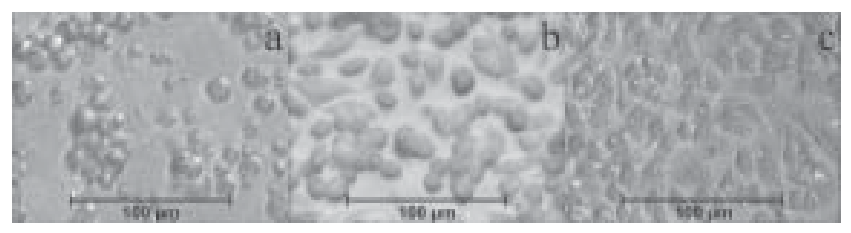

Figure 1: Mouse macrophage proliferation on (a) ingot processed 316L; (b) PM 316L; and (c) PM HNSS.

Preliminary results of a study of a mouse macrophage proliferation on SS substrates are shown in Figure 1. The spheroidized and necrotic cells on the ingot processed 316L substrate in Figure 1a indicate significant incompatibility; whereas the same cell line has adhered to the surface and proliferated on PM HNSS (Figure 1c), showing normal macrophage morphology. An intermediate result (some cells spherodized, others normal) was found on the PM 316L (Figure 1b). Compatibility studies on SS alloys will continue next year as well as including similar studies on the other two common implant alloys, Co-Cr-Mo and Ti-6Al-4V.

Another aspect of this project is the evaluation of the corrosion-pitting resistance of metallic implant materials. Previous NIST results indicate that PM HNSS has a remarkable resistance to pitting under a wide range of environmental conditions. While the observed enhancement may be the result of the high nitrogen content in these alloys, it could also be due to the fine grain size and improved homogeneity inherent to the rapid solidification process itself. To help separate these effects, electrochemical behavior of ingot processed 316L, PM 316L, and PM HNSS material is now being evaluated in a simulated human environment (i.e., Hank's solution at $37^{\circ} \mathrm{C}$ ). The results of the electrochemical evaluations will then be used to design a series of slow strain rate (SSR) tensile tests that will assess the susceptibility to stress corrosion cracking (SCC) under the same environmental conditions.

\section{Contributors and Collaborators}

R. Jiggetts, S. Mates, M. Stoudt (Metallurgy Division, NIST); L. Bailey, N. Washburn (Polymers Division, NIST); F. Eichmiller, S. Gruninger (ADA); J. Spicer, J. Lee (Johns Hopkins University); S. Ehrman, T. Holoman (University of Maryland); A. Fischer (University of Duisburg, Germany) 


\section{Materials for Micro- and Opto-Electronics}

U.S. microelectronics and related industries are in fierce international competition to design and produce smaller, lighter, faster, more functional, and more reliable electronics products more quickly and economically than ever before. At the same time, there has been a revolution in recent years in new materials used in all aspects of microelectronics fabrication.

Since 1994, the NIST Materials Science and Engineering Laboratory (MSEL) has worked closely with the U.S. semiconductor, component, packaging, and assembly industries. These efforts led to the development of an interdivisional MSEL program committed to addressing industry's most pressing materials measurement and standards issues central to the development and utilization of advanced materials and material processes. The vision that accompanies this program - to be the key resource within the Federal Government for materials metrology development for commercial microelectronics manufacturing - may be realized through the following objectives:

- Develop and deliver standard measurements and data;

- Develop and apply in situ measurements on materials and material assemblies having micrometer- and submicrometer-scale dimensions;

- Quantify and document the divergence of material properties from their bulk values as dimensions are reduced and interfaces contribute strongly to properties;

- Develop models of small, complex structures to substitute for or provide guidance for experimental measurement techniques; and

- Develop fundamental understanding of materials needed in future micro- and opto-electronics.

With these objectives in mind, the program presently consists of projects led by the Metallurgy, Polymers, Materials Reliability, and Ceramics Divisions that examine and inform industry on key materials-related issues. These projects are conducted in concert with partners from industrial consortia, individual companies, academia, and other government agencies. The program is strongly coupled with other microelectronics programs within government and industry, including the National Semiconductor Metrology Program (NSMP) at NIST. Materials metrology needs are also identified through industry groups and roadmaps, including the International Technology Roadmap for Semiconductors (ITRS), the IPC Lead-free Solder Roadmap, the National
Electronics Manufacturing Initiative (NEMI) Roadmap, the Optoelectronics Industry Development Association (OIDA) roadmaps, and the National [Magnetic Data] Storage Industry Consortium (NSIC).

Although there is increasing integration within various branches of microelectronics and optoelectronics, the field can be considered in three main areas. The first, microelectronics, includes needs ranging from integrated circuit fabrication to component packaging to final assembly. MSEL programs address materials metrology needs in each of these areas, including lithographic polymers and electrodeposition of interconnects, electrical, mechanical, and physical property measurement of dielectrics (interlevel, packaging, and wireless applications), and packaging and assembly processes (lead-free solders, solder interconnect design, thermal stress analysis, and co-fired ceramics).

The second major area is optoelectronics, which includes work which often crosses over into electronic and wireless applications. Projects currently address residual stress measurement in optoelectronic films, optical and structural characterization of wide bandgap semiconductors, and standards development for III-V compound semiconductors. Cross-laboratory collaborations with EEEL figure prominently in this work.

The third area is magnetic data storage, where the market potential is vast and growing and the technical challenges extreme. NSIC plans to demonstrate a recording density of 1 terabit per square inch - 40 times today's level — by 2006 . To reach these goals, new materials are needed that have smaller grain structures, can be produced as thin films, and can be deposited uniformly and economically. New lubricants are needed to prevent wear as spacing between the disk and head becomes smaller than the mean free path of air molecules. Some measurements require calibration of magnetometers using certified magnetic standards in several different shapes and magnetic strengths, and with a wide range in magnetic character. MSEL is working with the magnetic recording industry to develop measurement tools, modeling software, and standards to help achieve these goals, with MSEL, the Electronics and Electrical Engineering Laboratory, the Physics Laboratory, the Information Technology Laboratory, and the Manufacturing Engineering Laboratory working as partners in this effort.

Contact: Frank W. Gayle 


\section{Superconformal Film Growth: Measurement and Modeling}

State of the art manufacturing of microelectronic devices relies on superconformal deposition of copper in fine trenches and vias for on-chip wiring, or "interconnects." The NIST-developed Curvature Enhanced Coverage Mechanism is now used by industry to understand this superfilling process. More recent NIST research has demonstrated that the same mechanism explains the impact of catalysts on smoothing of surfaces during all types of electrodeposition. This is the first explanation of the surface stabilization process, a phenomenon that is as important for the filling of high aspect ratio features as the bottom-up superfill process itself.

\section{Thomas P. Moffat and Daniel Josell}

$\mathrm{T}$ his project continues to explore novel, advanced means of meeting needs of the microelectronics industry for improved device metallizations. Much of the effort focuses on the Curvature Enhanced Accelerator Coverage mechanism originally shown by NIST researchers to explain "superconformal" (or bottom-up) feature filling with copper. The CEAC mechanism accounts for the impact of changing surface area on the coverage of adsorbed additives used in electrodeposition processes. Studies of this mechanism continue to yield exciting new discoveries. One particular result serves especially well to demonstrate the breadth of the superfill studies and the striking generality of the CEAC mechanism.

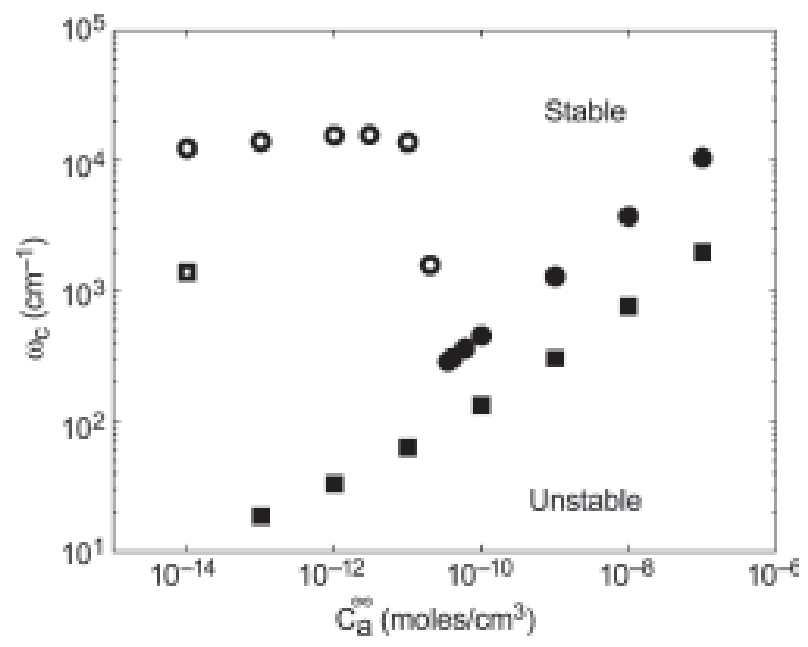

Figure 1: The impact of catalyst concentration in the electrolyte on the minimum wavenumber for which a perturbation is stable (circles and squares are for different overpotentials). Substantial improvement in the range of conditions for which the surface is stabilized (up to two-orders of magnitude decrease of the critical wavelength) is evident.
Researchers in the Metallurgy Division previously demonstrated the ability of the CEAC mechanism to explain key aspects of the bottom-up feature filling process that is known in the integrated circuit industry as "superfill." They have also extended it to superfill during electrodeposition of silver and chemical vapor deposition of copper. The CEAC mechanism has now been successfully extended to explain surface stabilization during electrodeposition of metals in electrolytes containing surfactant additives (Figure 1).

Such generality had been proposed in CEAC publications on feature superfill, but until this work, it had not been analyzed or proven. These results quantify the effect using a linear perturbation analysis; experimental evidence to support the model is also presented. Significantly, these results explain why remarkably dilute of additives known as "brighteners" or "accelerators" are so effective at preventing the roughening of surfaces even in the presence of destabilizing metal ion concentration gradients in the electrolyte.

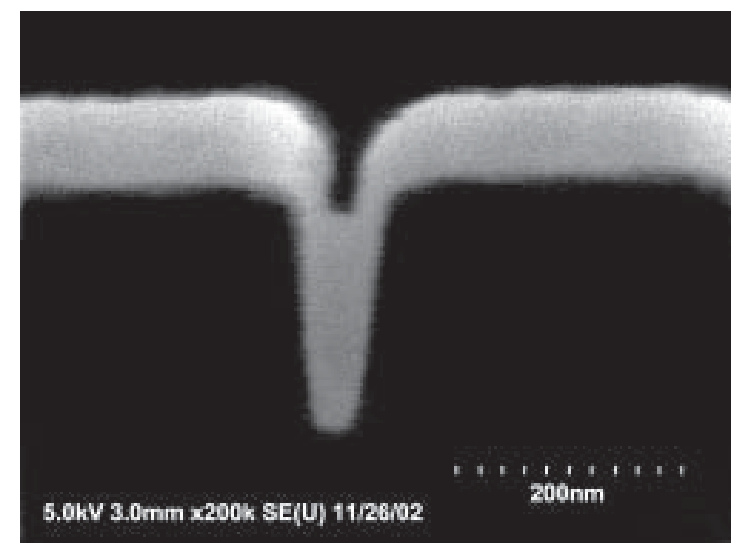

Figure 2: Superconformal copper electrodeposition. Trench width is approximately $50 \mathrm{~nm}$ at mid-height. Note the smooth sidewalls.

These results are of fundamental importance in the fabrication of high-aspect ratio advanced metallizations because smooth surfaces on the metal deposits are required for defect-free feature filling (Figure 2). Furthermore, these results provide understanding and direction in all electrodeposition processes providing brightening.

\section{Contributors and Collaborators}

D. Wheeler, S. Coriell, J. Mallett, W. Schwarzacher (Metallurgy Division, NIST); G. McFadden (Mathematical and Computational Sciences Division, NIST) 


\section{Lead-free Surface Finishes: Tin Whisker Growth}

The U.S. microelectronics industry is moving towards Pb-free assemblies but lacks needed measurements and data comparable to that existing for Sn-Pb alloys. Protective layers, such as "pretinned" coatings on Cu leadframes and connectors, are required to maintain solderability of components during storage prior to assembly. A critical problem addressed by this project is that $\mathrm{Pb}$-free coatings of nearly pure tin tend to grow "whiskers" which can cause catastrophic shorts across component leads.

\section{Maureen E. Williams, Christian Johnson, and Kil-Won Moon}

D evelopment of $\mathrm{Pb}$-free surface finishes is essential for the current move toward $\mathrm{Pb}$-free microelectronics assembly, and tests which ascertain the tendency to form "tin whiskers" are in demand. NIST is providing leadership in two National Electronics Manufacturing Initiative (NEMI) whisker projects: identifying fundamental growth mechanisms and developing an accelerated test. Our goal at NIST is to provide an understanding of the fundamentals of the whisker formation, which will ensure that the test developed by the NEMI group will have widespread and general applicability.

Filamentary Sn whiskers (typically $1 \mu \mathrm{m}$ diameter and several $\mathrm{mm}$ long) can grow from a Sn-based surface finish and cause electrical shorts and failure. Historically, $\mathrm{Pb}$ was added to $\mathrm{Sn}$ plate to prevent whisker growth as well as to lower cost. The current research program has focused on measuring effects of alloying additions on whisker formation in $\mathrm{Pb}$-free $\mathrm{Sn}$-rich deposits. The $\mathrm{Sn}-\mathrm{Cu}$ system was chosen since $\mathrm{Sn}-\mathrm{Ag}-\mathrm{Cu}$ is the $\mathrm{Pb}$-free solder of choice for industrial application. The goal is to substitute a different solute for $\mathrm{Pb}$ in the $\mathrm{Sn}$-rich deposit in order to retard whisker growth.

Residual stress is generally believed to drive whisker growth in electroplated Sn. However, the origin of this stress has not been determined definitively. Our analysis of pure $\mathrm{Sn}$ and $\mathrm{Sn}-\mathrm{Cu}$ deposits, combining a cantilever beam method for stress analysis with electron microscopy, indicates that $\mathrm{Cu}_{6} \mathrm{Sn}_{5}$ intermetallic particles in the $\mathrm{Sn}$ grains and along the grain boundaries of $\mathrm{Sn}-\mathrm{Cu}$ deposits inhibit stress relaxation (Figure 1). Coarse intermetallic particles decorate grain boundaries with finer particles within the grains. The compressive stress in the pure Sn deposit decreased from an initial level of $14 \mathrm{MPa}$ to zero after four days. In contrast, the initial compressive stress in the $\mathrm{Sn}-\mathrm{Cu}$ deposit was $32 \mathrm{MPa}$ and after twenty-three
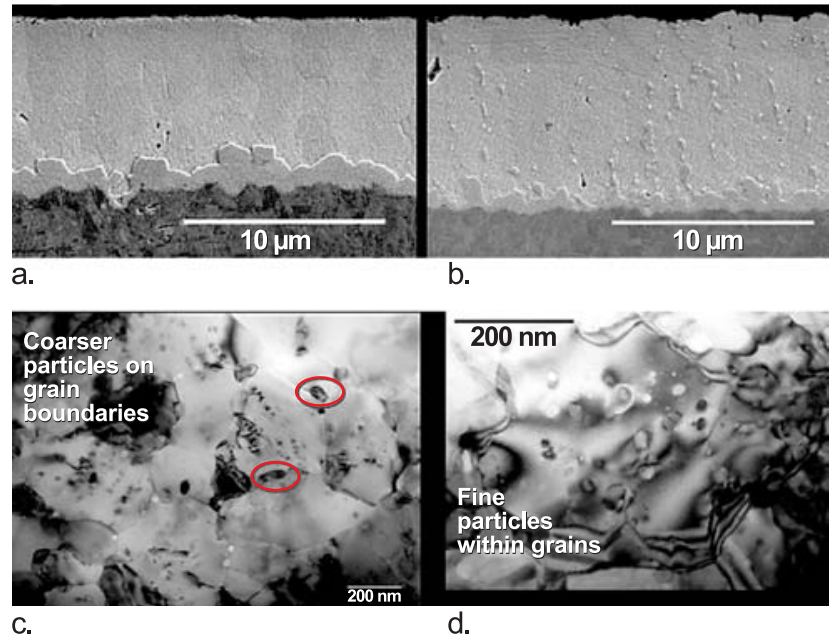

Figure 1: (a) Polished section of pure Sn on Cu substrate; (b) Polished section of SnCu alloy on Cu substrate; (c,d) TEM photos of intermetallic particles on grain boundaries and in Sn grains.

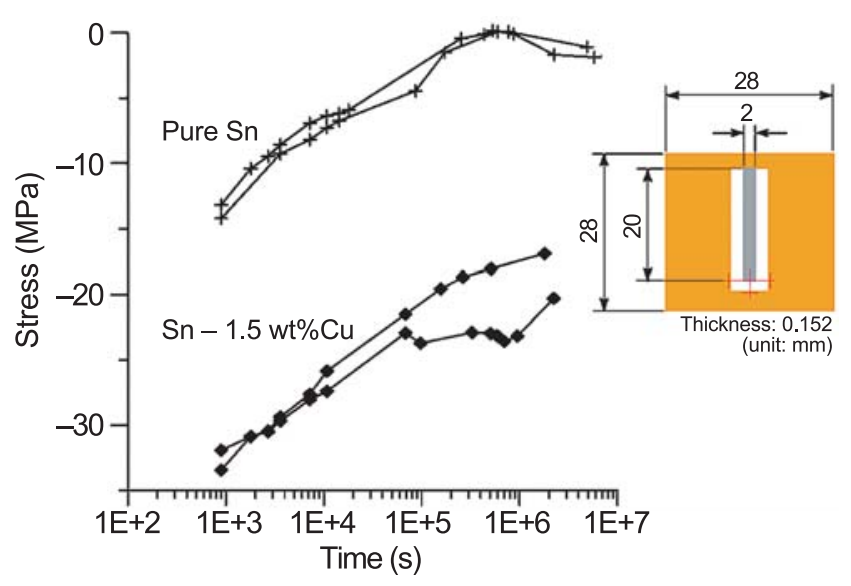

Figure 2: Calculated stress levels from cantilever beam measurements of pure Sn and Sn-Cu electrodeposits.

days was still $21 \mathrm{MPa}$ (Figure 2). Whiskers were present on the $\mathrm{Sn}-\mathrm{Cu}$ sample after only four days, but after 600 days, the pure Sn sample was still whisker-free. Since copper is a frequent impurity in electrodeposited Sn, unintendedly high, undocumented copper concentrations in commercial Sn deposits may have confounded controlled variables in previous studies. These results have been disseminated to the microelectronics community through NEMI meetings, including the March 2003 NEMI-NIST-TMS Workshop described in the Technical Highlights section.

\section{Contributors and Collaborators}

W.J. Boettinger, L. Bendersky, G.R. Stafford (Metallurgy Division, NIST); G. Galyon, N. Vo (NEMI project leaders) 


\section{Lead-Free Solders and Solderability}

Solders and solderability are increasingly tenuous links in the assembly of microelectronics as a consequence of ever-shrinking chip and package dimensions and the international movement toward environmentally friendly lead-free solders. In collaboration with the NEMI Pb-Free Assembly Project, we are providing the microelectronics industry with measurement tools, data, and analyses that address national needs in the implementation of lead-free solders.

\section{Ursula Kattner and Carol Handwerker}

Since 1999, NIST has served a major role in the National Electronics Manufacturing Initiative (NEMI) Pb-Free Assembly Project to identify and move $\mathrm{Pb}$-free solders into the microelectronics industry. (NEMI is an industry-led consortium of 65 manufacturers, suppliers, and related organizations brought together to facilitate leadership of the North American electronics manufacturing supply chain.) This move toward $\mathrm{Pb}$-free solders is a direct result of the European Union ban, starting in 2006, on $\mathrm{Pb}$-containing solders in electronic products.

The NEMI Lead-free Task Force and NIST have worked together to respond to the identified needs by:

- Identifying and providing the most important lead-free solder data for the microelectronics community, including the definitive database on thermodynamic properties of $\mathrm{Pb}$-free alloys, and dessiminating such data on the NIST website. (See "Informatics and Visualization in Materials Data Delivery" in this volume.) This database allows the calculation of phase transformations in solder alloys containing $\mathrm{Ag}, \mathrm{Cu}, \mathrm{Bi}, \mathrm{In}, \mathrm{Pb}, \mathrm{Sb}$, and $\mathrm{Sn}$, with additional data for $\mathrm{Ag}, \mathrm{Cu}$, and $\mathrm{Sn}$ with $\mathrm{Ni}$, which is used in characterizing interactions between $\mathrm{Pb}$-free solders and Ni-based surface finishes.

- Developing and widely disseminating "Recommended Practice Guide on Test Procedures for Developing Solder Data." (See Figure 1.)

- Providing a list of literature references on alloys, processing, reliability, environmental issues, and components for the implementation of lead-free solders.

- Completing the microstructure-based failure analysis on all thermally cycled assemblies as part of the NEMI's project's full-scale reliability trials.

- Providing chapters on the materials science of $\mathrm{Pb}$-free solder alloys for three books on $\mathrm{Pb}$-free solders.
In 2003, we have also served as an information resource to the microelectronics industry on issues related to the effects of alloy composition, reflow temperature, and furnace profiles for best melting and solidification behavior of solders when circuit boards are assembled, and to the effects of $\mathrm{Pb}$ contamination on alloy melting and assembly reliability.

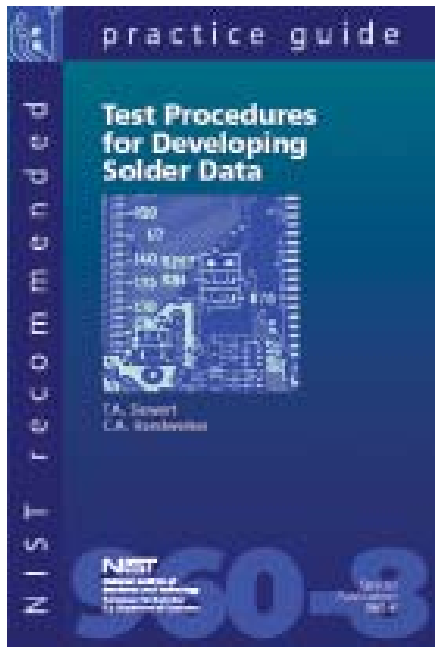

Figure 1: "NIST

Recommended Practice Guide on Test Procedures for Developing Solder Data."

"The NIST group performed a significant service to industry by being the focal point for development of a reliable technology base to support the choice of a new lead-free alloy. They also led the way for further work on issues such as tin whiskers, which emerged from the initial lead-free work. NIST provided not only a strong technical basis but, by verifying reliability and comparing alternatives, enabled industry to choose an alloy based on extensive investigation. The prompt global establishment of a preferred alloy solution was critical for Motorola to be an early adopter of the technology."

Dr. Robert C. Pfahl, Jr., Director International and Environmental R\&D Motorola, (retired)

\section{Contributors and Collaborators}

W.J. Boettinger, K.-W. Moon, L.E. Smith, S.W. Claggett, M.E. Williams (Metallurgy Division, NIST); T. Siewert, C. McCowan (Material Reliability Division, NIST); J.P. Clech (EPSI, Inc.); J. Bath (Solectron); R. Gedney (NEMI); E. Bradley (Motorola); J.E. Sohn (NEMI, formerly Lucent); P. Snugovsky (Celestica); E. Benedetto (HP); R. Charbonneau (StorageTek) 


\section{Magnetic Properties and Standard Reference Materials}

Users of magnetic materials, including the recording industry, the permanent magnet industry, and the manufacturers of electric motors and transformers of all types, need standard reference materials (SRM's) to calibrate instruments used to measure the critical magnetic properties of their starting materials. The Magnetic Materials Group develops such standards. We also investigate methods for improving the efficiency and accuracy of measuring and characterizing magnetic materials of industrial interest.

\section{Robert D. Shull and Robert D. McMichael}

Tn collaboration with scientists from universities, industry, and other Divisions at NIST, this project prepares magnetic materials that are important to the scientific and industrial communities and develops methods for the improved measurement of their properties. We develop models of hysteresis and magnetic time dependence that serve as a theoretical foundation for metrology of accommodation and aftereffect in magnetic recording and permanent magnetic materials. We also develop models for determination of the most efficient methods to fully characterize the hysteresis and magnetostriction in magnetic materials.

We develop and produce Standard Reference Materials for the calibration of existing and planned instruments used in the measurement of magnetic properties using the absolute magnetometer developed in our laboratory (e.g., Figure 1). This year we have issued garnet magnetic moment SRM's useful for calibrating the most sensitive scales in SQUID and vibrating sample magnetometers. These SRM's provide, for the first time, a means for calibrating the measurements from high sensitivity alternating gradient magnetometers on an absolute basis. These small (1 $\mathrm{mm}$ diameter) single-crystal yttrium iron garnet spheres with a nominal mass of about $2.8 \mathrm{mg}$ were measured using the Faraday technique after calibrating the magnetometer using the Thorpe-Sentfle method. Current work includes certifying platinum cylinders as paramagnetic susceptibility standards for the magnetics community. This standard reference material will be useful for calibrating magnetometers where an outside source of magnetic flux is undesirable. It will also be useful for determining the true zero field and zero moment values in magnetometers.

In order to inform the magnetics community of the availability of these recently prepared magnetic standard reference materials, a NIST booth on this topic was

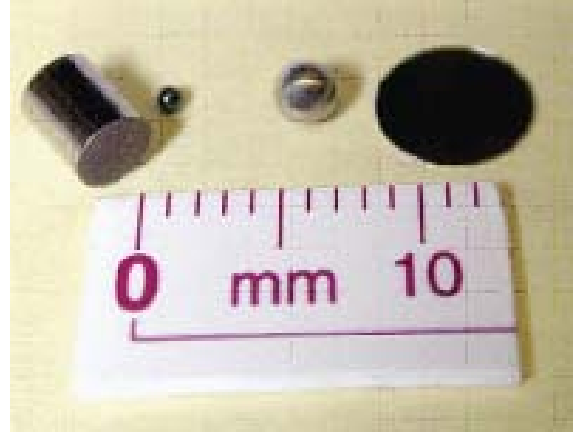

Figure 1: Pt cylinder, YIG sphere, Ni sphere, and Ni disc SRMs.

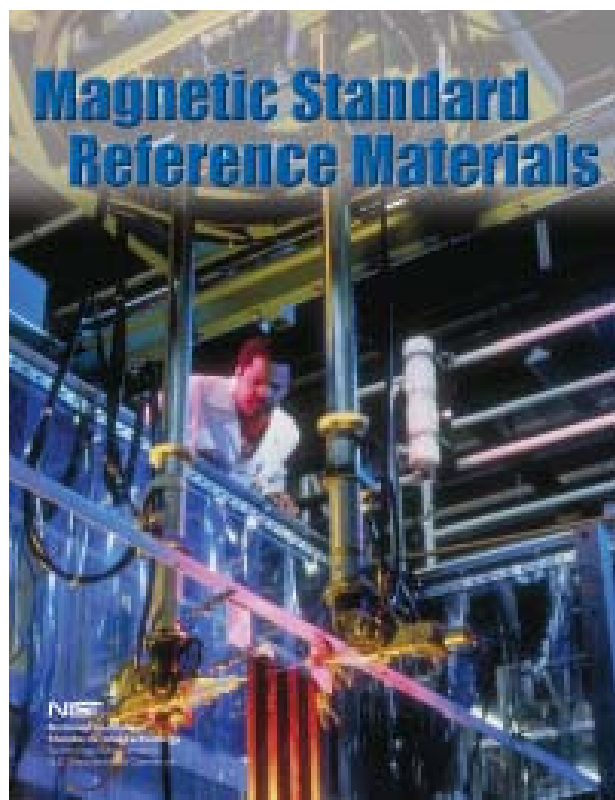

Figure 2: Magnetic SRM brochure.

organized and manned at the last Magnetism and Magnetic Materials Conference (MMM, the big annual conference on magnetism in the U.S.). In addition, a brochure was assembled in collaboration with the Measurement Sciences Division of NIST, shown in Figure 2, and mailed to over 7,000 magneticians, with an electronic version sent by email to the participants of the above MMM conference.

\section{Contributors and Collaborators}

L.J. Swartzendruber (Magnetronix, Inc.); D. Twisselmann (Arete Associates, Inc.); D.Mathews, R. Drew (Metallurgy Division, NIST); S.D. Leigh (Statistical Engineering Division, NIST); D. Decker (Measurement Services Division, NIST); L. Bennett, E. Della Torre (George Washington University) 


\section{Magnetic Properties of Nanostructured Materials}

In the past 10 to 15 years, there has been a remarkable improvement in the technology for materials preparation, resulting in today's capability of controlling morphology and features at the nanometer level. In magnetic materials, such control allows the fabrication of $n$ m-thick (or separated) composite materials of dissimilar magnetism, leading to materials with novel magnetic character and unusual property combinations. We provide the understanding and metrology that allows U.S. industry to take advantage of these new materials.

\section{Alexander J. Shapiro and Robert D. Shull}

$\mathrm{P}$ resent day high-density data storage is possible due to the giant magnetoresistance (GMR) spin valve, which is now used as the read head in magnetic disc drives. This structure is composed of two ferromagnetic (FM) layers separated by a thin non-magnetic layer. When an external magnetic field reverses the magnetization of one of the FM layers, the resistance of the composite changes. Consequently, one FM layer must maintain a constant magnetization during this process, usually accomplished by placing an antiferromagnetic (AF) layer adjacent to it so as to "exchange-bias" that FM layer. This results in a hysteresis loop that is shifted along the field axis as shown in Figure 1(a). Unfortunately, this exchange-biasing is not well understood.

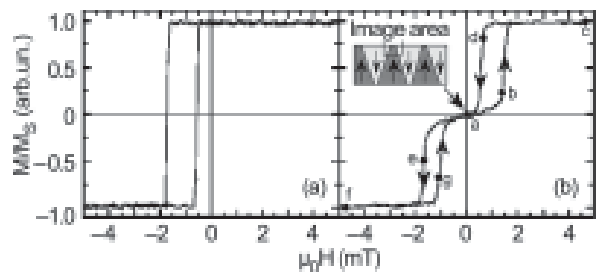

Figure 1: Hysteresis loops for a NiFe/FeMn bilayer cooled under (a) constant and (b) alternating field conditions.

Several years ago, we found we could elucidate some of the basics of the magnetization reversal process in such systems [V.I. Nikitenko, et. al., PRB 57, R8111 (1998)] by using the magneto-optic indicator film technique (MOIF) developed in our laboratory. This domain imaging technique enabled us to show for the first time that, contrary to popular thought, the two sides of the hysteresis loop are not symmetrical but are each governed by different mechanisms. This year, we have answered another key question regarding exchanged bias systems: do the domain walls in the AF move when the FM reverses its magnetization? MOIF observations were made of a NiFe (FM) / FeMn (AF) bilayer which had been ac-demagnetized above the
Neel temperature of the AF but below the Curie point of the FM (Figure 1b). Application of the alternating field at elevated temperatures formed a demagnetized FM composed of striped domains with alternating magnetization vectors, shown in Figures 1(b) and 2(a), on top of coincident AF domains. When a field was applied to this bilayer to saturate the FM [Figures 2(c), (f)], there still remained a remnant image of the original domain pattern (Figure 2a), indicating the $\mathrm{AF}$ wall below the FM has not moved.

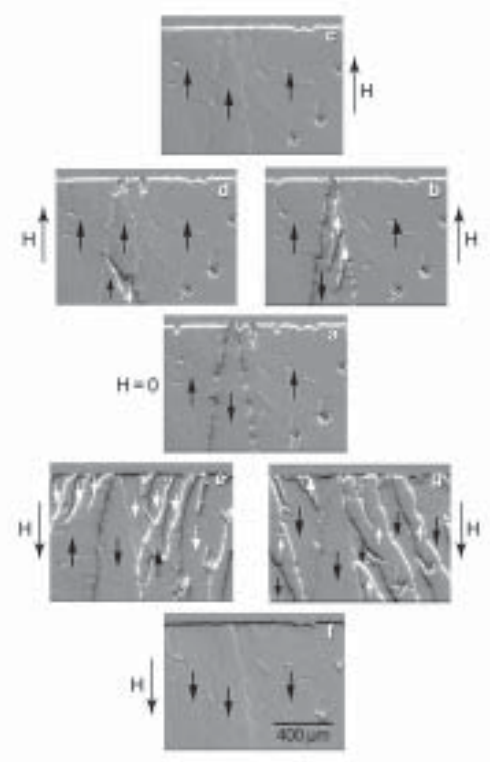

Figure 2: MOIF images of domains in the FM at the conditions specified by the positions designated in Figure 1(b).

Some AF spins in the region of the AF/FM interface, however, are changing during the FM reversal, as attested to by the fact that Figures 2(e) and (g) only show domain activity in the same areas for both field application and removal respectively, contrary to that for a normal ferromagnet. Similar observations have also been made on a different AF/FM bilayer, FeMn/ $\mathrm{Fe}_{76} \mathrm{Mn}_{6} \mathrm{C}_{18}$, wherein the initial coincident domain structure in the FM and AF was created by elevated temperature preparation under a low applied field.

As a result of these measurements, theoretical models may now be improved, and industry should now be able to better tailor spin valve processing to obtain improved reproducibility. In the past year, over $40 \%$ of all papers on exchange-biasing have referenced our MOIF results.

\section{Contributors and Collaborators}

V. Nikitenko, V. Gornakov (Russian Academy of Sciences); C.L. Chien (Johns Hopkins University); H.-W. Zhao (Chinese Academy of Sciences) 


\section{Nanomagnetodynamics}

This project provides the magnetic information storage industry with metrology for magnetization damping and magnetic inhomogeneity in thin film ferromagnets. Damping is expected to become a critical issue for magnetic information storage at $\mathrm{GHz}$ data rates, and inhomogeneity on the scale of $10 \mathrm{~nm}$ in recording media is important for increased storage density in the new generation of disk drives, which will use perpendicular magnetic recording.

\section{Robert D. McMichael}

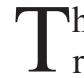
he central issue in interpretation of ferromagnetic resonance line width data is the determination of how much of the ferromagnetic resonance line width is due to damping and how much is due to inhomogeneity. Ferromagnetic resonance is observed as a peak in the transverse susceptibility that occurs when the frequency of the driving field is close to the frequency of precession. The peak position can be used to determine sample average properties, but the width of the resonance peak, or "line width," is a combined effect of damping and inhomogeneity.

Existing simple models describe the contribution of damping to the line width. We have provided reliable models of inhomogeneity that are key to interpretation of line width data. These models describe the line

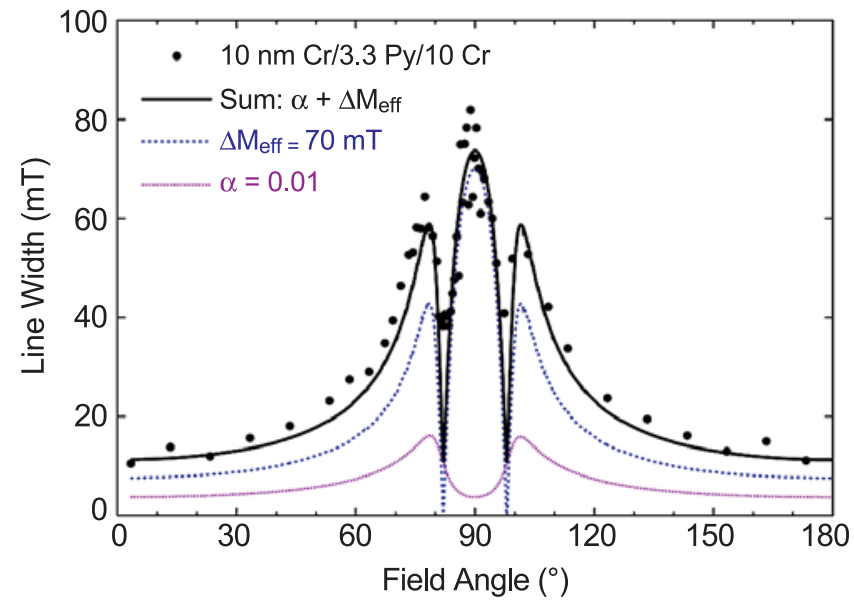

Figure 1: Ferromagnetic resonance linewidth for a $\mathrm{Cr} /$ Permalloy/Cr trilayer as a function of applied field angle. This plot illustrates the ability to separate the linewidth into a damping component (pink line) and an inhomogeneous component (blue line) corresponding to $7 \%$ variations in the effective magnetization. width behavior as a function of frequency and applied field orientation for different types of inhomogeneity.

Neglecting the exchange and dipolar interactions that are characteristic of ferromagnets, local variations in magnetic properties would be expected to result in a distribution of local precession frequencies and a broadened resonance peak. However, interactions tend to synchronize the precession in neighboring regions, effectively "smoothing" the inhomogeneity, and reducing its effect on the line width.

Significant accomplishments in FY03 include:

- Development of a unified model of inhomogeneous line broadening in thin films. This model covers the full range of inhomogeneity and interaction strengths but is computationally too expensive to use for fitting. This model established that the transition from local to collective behavior is governed by the relative magnitudes of the inhomogeneity and the region-to-region coupling. See R.D. McMichael, D.J. Twisselmann, and Andrew Kunz, Phys. Rev. Lett., 90, Art. No. 277601 (2003).

- An approximation to the full calculation of inhomogeneous line broadening using the "two magnon" model in the strong coupling/weak inhomogeneity limit and local resonances in the weak coupling/strong inhomogeneity limit.

- Useful analytical approximations for the "two magnon" model for ultra thin films in the limit of zero damping.

- Measurement of the damping properties of $\mathrm{Cr}$ in proximity to Permalloy (Figure 1). These experiments imply that ferromagnetic resonance measurements in $\mathrm{CoCr}$ based hard drive recording media will not be hindered by excessive damping.

- Modeling of ferromagnetic resonance line widths in perpendicular recording media under development in industry.

\section{Contributors and Collaborators}

D.J. Twisselmann, W.F. Egelhoff, Jr., A.P. Chen (Metallurgy Division, NIST); Andrew Kunz (Lawrence University); H. Nakamura, M.E. McHenry (CMU); S. Rezende (Recife, Brazil); G.S.D. Beach, A.E. Berkowitz (UCSD) 


\section{Anomalously Large Low Temperature Intermixing in Metallic Thin Films}

The objective of this program is to provide assistance to U.S. companies in the ultra-high density data storage industry, which includes such products as hard disk drives and magneto-resistive random access memory chips. Our work provides U.S. companies with significant competitive help by investigating the scientific issues underlying the manufacturing process. Often, this scientific understanding will point the way to improved manufacturing processes.

\section{William F. Egelhoff, Jr.}

$\mathrm{A}^{\mathrm{s}}$ $\mathrm{s}$ the scale of microelectronic devices shrinks, surfaces and interfaces play increasingly important roles as they become a larger fraction of the total device volume. Interfaces are especially important for the metallic thin films so common in layered device structures. Interfaces between thin films may appear to be abrupt at macroscopic length scales but diffuse at atomic length scales. Fortunately, when metallic thin films are deposited on one another at room temperature, there is generally little intermixing at the interface. Typically, if there is any intermixing, it is limited to one or two atomic layers. However, recent collaborative studies by NIST and the University of Durham have shown that such low-temperature intermixing can be far more extensive when $\mathrm{Al}$ and a transition metal (TM) are involved.

Thin film samples of the type "Al on TM" and "TM on Al" were prepared at the NIST Magnetic Engineering Research Facility and analyzed by grazing-incidence $\mathrm{x}$-ray reflectometry (GIXR) at Durham. GIXR is an ideal technique for these studies since it is extremely sensitive to intermixing at interfaces. We find the intermixing profile perpendicular to the interface is usually well described by a Gaussian distribution, as expected, since diffusion processes often follow Fick's Law. The results are displayed in Table 1 as the full-width-at-half-maximum (FWHM) of the intermixed region. The FWHM results span the range from $0.1 \mathrm{~nm}$ for $\mathrm{Al}$ on $\mathrm{W}$ to $16.8 \mathrm{~nm}$ for $\mathrm{Cu}$ on $\mathrm{Al}$. This $\mathrm{Cu}$-on- $\mathrm{Al}$ result corresponds to approximately 100 atomic layers of intermixing. Such results are a great surprise to researchers in the field. For example, electronic properties for a thin film structure consisting of $2 \mathrm{~nm} \mathrm{Cu} / 1 \mathrm{~nm} \mathrm{Al} / 2 \mathrm{~nm} \mathrm{Cu}$ were recently reported and analyzed as if the interfaces were atomically sharp. In fact, the sample must have been essentially a random alloy!
Among other combinations showing large intermixing were $\mathrm{Mn}$ on $\mathrm{Al}$ with $15.1 \mathrm{~nm}$ and $\mathrm{V}$ on $\mathrm{Al}$ with $9.4 \mathrm{~nm}$. These surprising results suggest that the heat of alloying is driving much more extensive chemical reactions at the interface than had heretofore been known. These results have important implications for many microelectronic devices incorporating $\mathrm{Al}$ films. For example, problems in magnetic-tunnel-junction devices may be directly attributed to such phenomena. NIST is also developing solutions to such problems based on oxide barriers to suppress the intermixing.

Table 1: The intermixing FWHM (in nanometers) for thin films of Al on transition metals and transition metals on Al

\begin{tabular}{ccc} 
TM Element & Al on TM & TM on Al \\
\hline $\mathrm{Ti}$ & 1.7 & 5.0 \\
$\mathrm{~V}$ & 2.6 & 9.4 \\
$\mathrm{Cr}$ & 0.5 & 3.3 \\
$\mathrm{Mn}$ & 10.4 & 15.1 \\
\hline $\mathrm{Fe}$ & 0.9 & 2.1 \\
$\mathrm{Co}$ & 0.8 & 6.8 \\
\hline $\mathrm{Ni}$ & 1.4 & 7.9 \\
$\mathrm{Cu}$ & 2.8 & 16.8 \\
$\mathrm{Zr}$ & 1.0 & 5.1 \\
$\mathrm{Nb}$ & 0.8 & 3.6 \\
\hline $\mathrm{Mo}$ & 1.3 & 3.4 \\
$\mathrm{Ru}$ & 0.8 & 5.2 \\
\hline $\mathrm{Rh}$ & 0.4 & 4.7 \\
$\mathrm{Pd}$ & 4.8 & 5.6 \\
\hline $\mathrm{Ag}$ & 2.5 & 4.5 \\
$\mathrm{Hf}$ & 2.0 & 4.4 \\
\hline $\mathrm{Ta}$ & 0.1 & 0.9 \\
$\mathrm{~W}$ & 0.1 & 3.5 \\
\hline $\mathrm{Re}$ & 2.1 & 8.6 \\
\hline $\mathrm{Os}$ & 0.1 & 7.1 \\
\hline $\mathrm{Ir}$ & 0.2 & 5.4 \\
\hline $\mathrm{Pt}$ & 1.9 & 4.5 \\
\hline $\mathrm{Au}$ & 5.2 & 6.3 \\
\hline
\end{tabular}

\section{Contributors and Collaborators}

R.D. McMichael (Metallurgy Division, NIST); M.D. Stiles (Electron and Optical Physics Division, NIST); C.J. Powell (Surface and Microanalysis Science Division, NIST); Readrite Corporation; Seagate Corporation; J.D.R. Buchanan (Durham, UK); J. Moodera (MIT) 


\section{Materials Property Measurements}

This program responds both to the Materials Science and Engineering Laboratory (MSEL) customer requests and to the Department of Commerce 2005 Strategic Goal of "providing the information and framework to enable the economy to operate efficiently and equitably." For example, manufacturers and their suppliers need to agree on how material properties should be measured. Equally important, engineering design depends on accurate property data for the materials that are used.

The MSEL Materials Property Measurement Program works toward solutions to measurement problems on scales ranging from the macro to the nano, in four of the Laboratory's Divisions (Ceramics, Materials Reliability, Metallurgy, and Polymers). The scope of its activities ranges from the development and innovative use of state-of-the-art measurement systems, to leadership in the development of standardized test procedures and traceability protocols, to the development and certification of Standard Reference Materials (SRMs). A wide range of materials is being studied, including polymers, ceramics, metals, and thin films (whose physical and mechanical properties differ widely from the handbook values for their bulk properties).

Projects are directed toward innovative new measurement techniques. These include:

- Measurement of the elastic, electric, magnetic, and thermal properties of thin films and nanostructures (Materials Reliability Division);

- Alternative strength test methods for ceramics, including cylindrical flexure strength and diametral compression (Ceramics Division); and

The MSEL Materials Property Measurement Program is also contributing to the development of test method standards through committee leadership roles in standards development organizations such as the American Society for Testing of Materials (ASTM) and the International Standards Organization (ISO). In many cases, industry also depends on measurements that can be traced to NIST Standard Reference Materials (SRMs). This program generates the following SRMs for several quite different types of measurements:

- Charpy impact machine verification (Materials Reliability Division);

- Hardness standardization of metallic materials (Metallurgy Division); and

- Hardness standardization and fracture toughness of ceramic materials (Ceramics Division).

Supporting the Materials Property Measurements Program is a modeling and simulation effort to connect microstructure with properties. The Object-Oriented Finite-Element (OOF) software developed at NIST is being used widely in diverse communities for material microstructural design and property analysis at the microstructural level.

In addition to the activities above, all four divisions provide assistance to various government agencies on homeland security and infrastructure issues. Projects include assessing the performance of structural steels as part of the NIST World Trade Center Investigation, advising the Bureau of Reclamation on metallurgical issues involving pipelines and dams, advising the Department of the Interior on the structural integrity of the U.S.S. Arizona Memorial, advising the U.S. Customs Service on materials specifications for ceramics, and advising the Architect of the Capitol on repair procedures for cracks in the outer skin of the Capitol Dome.

Contact: Sam R. Low 


\section{Analysis of Structural Steel in the World Trade Center}

In 2002, NIST became the lead agency in a planned investigation of the World Trade Center collapse. The investigation addresses many aspects of the catastrophe, from occupant egress to factors affecting how long the Twin Towers stood after being hit by the airplanes, with a goal of gaining valuable information for the future. A critical aspect of the investigation is the metallurgical and mechanical analysis of structural steels from the Twin Towers.

The analysis includes characterization of properties, failure modes, and temperature excursions seen by the steel.

\section{Steve W. Banovic, William E. Luecke, Tim Foecke, Richard J. Fields, and Frank W. Gayle}

$\mathrm{T}$ he collapse of the twin World Trade Center (WTC) Towers on September 11, 2001, was the worst building disaster in human history. Engineers, emergency responders, and the nation were largely unprepared for such a catastrophe. These events highlight the following national needs:

- To establish the probable technical causes of the collapses and derive the lessons to be learned;

- To develop and disseminate immediate guidance and tools to assess and reduce future vulnerabilities; and

- To produce the technical basis upon which cost-effective changes to national practices and standards can be developed.

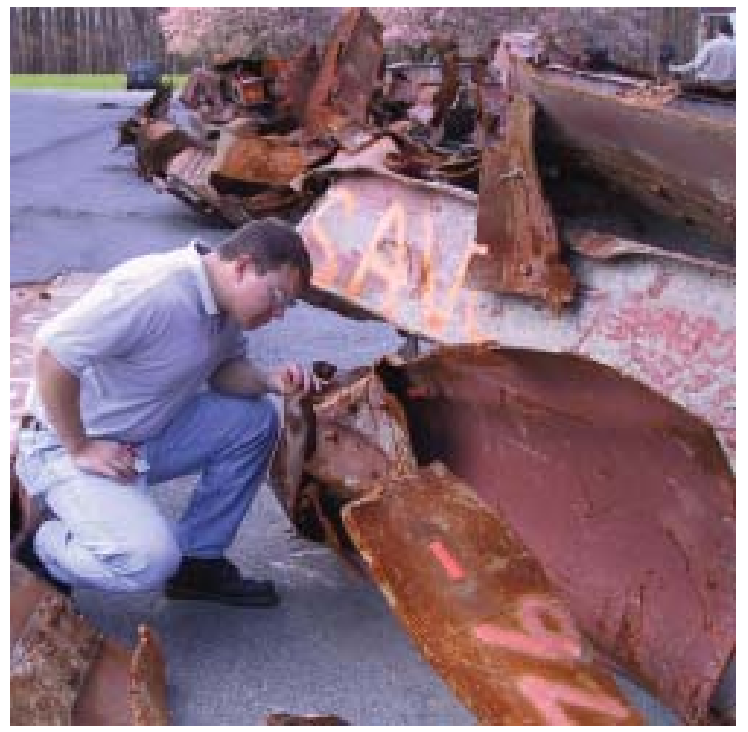

Figure 1: Failure mechanisms observed in recovered WTC steel will be used to gain insight into the building collapse.
NIST has prepared a technical plan to address these issues (see http://wtc.nist.gov/). A primary objective of the investigation is to determine why and how the towers collapsed after the initial impact of the aircraft. As part of this investigation, the Metallurgy and Materials Reliability Divisions in MSEL are studying recovered structural steel pieces from the WTC site. Progress in this study is outlined here.

Task 1 - Collect and catalog physical evidence. More than 200 pieces of the World Trade Center towers have been collected and brought to NIST. The locations of 41 exterior column panels within the WTC towers have been positively identified, of which 15 were located in or near the impact zones of the aircraft. Specified strengths of structural components have been identified through engineering drawings for the buildings.

Task 2 - Categorize failure mechanisms based on visual evidence. Recovered steel is being examined and documented as to failure mechanisms (Figure 1).

Task 3 - Determine steel properties to support structure performance and airplane impact modeling studies. Fourteen grades of steel were specified in the WTC towers. All grades have been characterized for room temperature mechanical properties, and initial high-temperature test results are complete. High strain rate testing is underway to determine the effects of strain rate on the strength of key structural elements. Chemical composition and metallographic examinations have been completed on the majority of the steels. Creep, or time-temperature-dependent behavior, will be studied after the high-temperature properties are developed.

Task 4 - Correlate determined steel properties with specified properties. As data are generated during the testing phase of the investigation, measured values of strength are compared to those specified in the engineering drawings.

Task 5 - Analyze steel to estimate temperature extremes. Microscopic, macroscopic and metallographic analyses are under way to determine the maximum temperature excursions seen by the steel.

An interim report, containing data generated to date, will be released to aid in the modeling of the aircraft impact and building response to subsequent fires. A final report will be complete at the end of FY04.

\section{Contributors and Collaborators}

D. McColskey, T. Siewert, R. Santoyo, L. Rodine (Materials Reliability Division, NIST) 


\section{Hardness Standardization: Rockwell, Vickers, Knoop}

Hardness is the primary test measurement used to determine and specify the mechanical properties of metal products. The Metallurgy Division is engaged in all levels of standards activities to assist U.S. industry in making hardness measurements compatible with other countries around the world. These activities include the standardization of the national hardness scales, development of primary reference transfer standards, leadership in national and international standards writing organizations, and interactions and comparisons with various U.S. laboratories and the National Metrology Institutes of other countries.

\section{Sam R. Low and Christian E. Johnson}

\begin{abstract}
$\mathrm{A}^{\mathrm{t}}$ the international level, we are leading the Working Group on Hardness (WGH) under the International Committee for Weights and Measures (CIPM). The primary goal is to standardize hardness measurements worldwide. As secretary of the WGH, we have lead an effort this year to better define the Rockwell hardness test procedure used by National Metrology Institutes (NMIs). Also, NIST's capabilities for hardness calibrations have recently been accepted into the CIPM Mutual Recognition Arrangement (MRA), an international document providing a means for comparing the measurements and calibrations of the world's NMIs. Other activities include heading the U.S. delegation to the ISO committee on hardness testing of metals, which oversees the development of the ISO hardness test method standards and is the Secretariat for the International Organization of Legal Metrology (OIML) committee on hardness, and we have proposed a revision of international requirements for regulating Rockwell hardness machines.
\end{abstract}

Our primary task at the national level is to standardize the U.S. national hardness scales and to provide a means of transferring these scale values to industry. Currently, we are producing test block Standard Reference Materials ${ }^{\circledR}$ (SRMs) for the Rockwell, Vickers and Knoop hardness scales, as well as developing new reference standards. Twelve microhardness SRMs for Vickers and Knoop hardness are now available, including two new nickel Vickers SRMs. Using electrodeposition technology, the standards are produced with uniform properties and microstructure. One hundred thirty-five SRMs were produced and certified to fill out-of-stock inventory. Work on a prototype high hardness Vickers microhardness SRM $(\approx 800)$ was redirected from an electrodeposited amorphous material to an evaluation of uniformity in microhardness values for Rockwell 63 HRC SRM test blocks at a load of $4.9 \mathrm{~N}$ (500 gf).
We also completed the calibration of two new SRMs for the Rockwell B scale (HRB) to complement the three SRM Rockwell C scale blocks currently available. The HRB scale is used for testing softer metals, such as aluminum, copper and brass. Work is also being planned to produce a new Rockwell diamond indenter SRM, which has become feasible due to the successful completion of a Small Business Innovative Research (SBIR) Phase-2 project with Gilmore Diamond Tools to develop an improved method for manufacturing geometrically correct Rockwell diamond indenters.

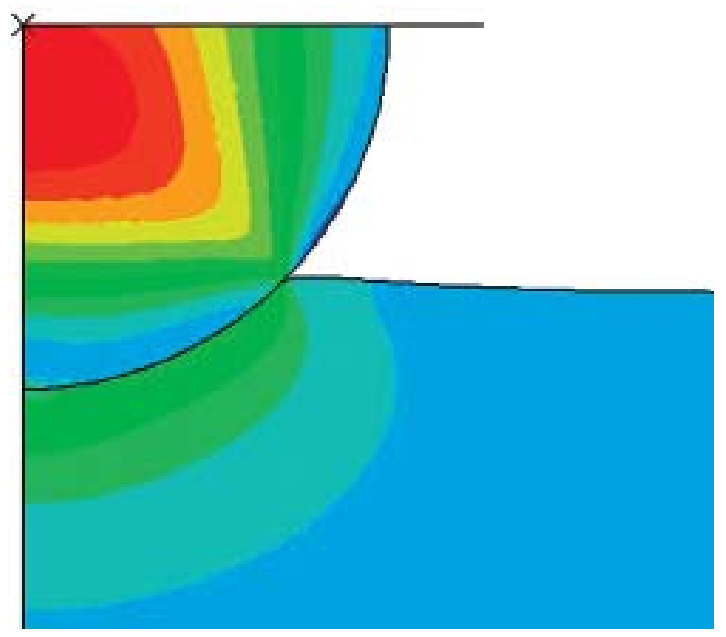

Figure 1: Model of stresses and material deformation occurring during Rockwell hardness ball indentation.

Other activities at the national level included: the assessment of commercial secondary hardness calibration laboratories for the NIST National Voluntary Laboratory Accreditation Program (NVLAP) providing direct linkage to the use of the NIST SRMs; development of a new method for estimating hardness uncertainty that has been accepted for inclusion into the ASTM-International Rockwell hardness test method standard E18; and development of indentation software models (Figure 1) that have been used to estimate the effect of varying test parameters on the resulting hardness value.

\section{Contributors and Collaborators}

J. Fink, D. Kelley, L. Ma, H. Gates (Metallurgy Division, NIST); J. Song, C. Evans, R. Clary (Fabrication Technology Division, NIST); W. Liggett, Jr., N. Zhang (Statistical Engineering Division, NIST); S. Doty, B. Belzer, D. Faison (Standards Services Division, NIST);

B. MacDonald (Measurement Services Division, NIST); W. Stiefel (Weights and Measures Division, NIST); M. Mihalec (Gilmore Diamond Tools, Providence, RI) 


\section{Marine Forensics and Preservation of Historic Shipwrecks}

Historic shipwrecks are valuable cultural resources continuously under attack by their environment. Preservation techniques aimed at slowing corrosion processes and mechanically stabilizing shipwreck structures are being modeled and designed in coordination with the National Park Service, NOAA, and the U.S. Navy. These techniques are being applied to such wreck sites as the USS Arizona, the Ellis Island Ferry, RMS Titanic, CSS Hunley, and USS Monitor.

\section{Tim Foecke}

Since sinking at Pearl Harbor on December 7, 1941, the wreck of the USS Arizona has been slowly corroding and collapsing (Figure 1). The monument that straddles the wreck sees over 700,000 visitors per year, and there is immense interest in preserving the wreck for as long as possible. Aside from the historical interest, there are environmental risks associated with any large-scale collapse of the wreck as there is still an estimated 2,000 $\mathrm{m}^{3}(500,000 \mathrm{gal})$ of fuel oil trapped below water.

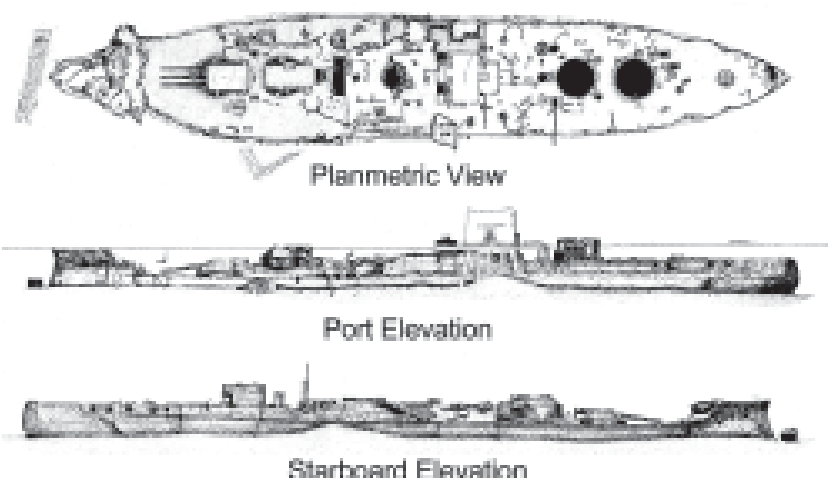

Figure 1: Current state of the USS Arizona in Pearl Harbor (from NPS Submerged Cultural Resources Unit).

The Submerged Cultural Resources Unit (SCRU) of the National Park Service has responsibility for maintaining and preserving the wreck and approached the Metallurgy Division for assistance, knowing their past experience in marine forensics. Assistance is being provided in several areas. Advice is being given on methods to be employed to measure the remaining thickness of the hull through the bioencrustation, and characterization of the steel and the encrustation layer is being performed.

This information is being input, along with the physical survey of the wreck, into a finite element model that will be used to predict the stresses in the wreck and how these stresses will build toward a critical state as the wreck continues to corrode. Using this model, various preservation techniques such as cathodic protection and physical reinforcements will be tried in the computer before anything is implemented on the wreck.

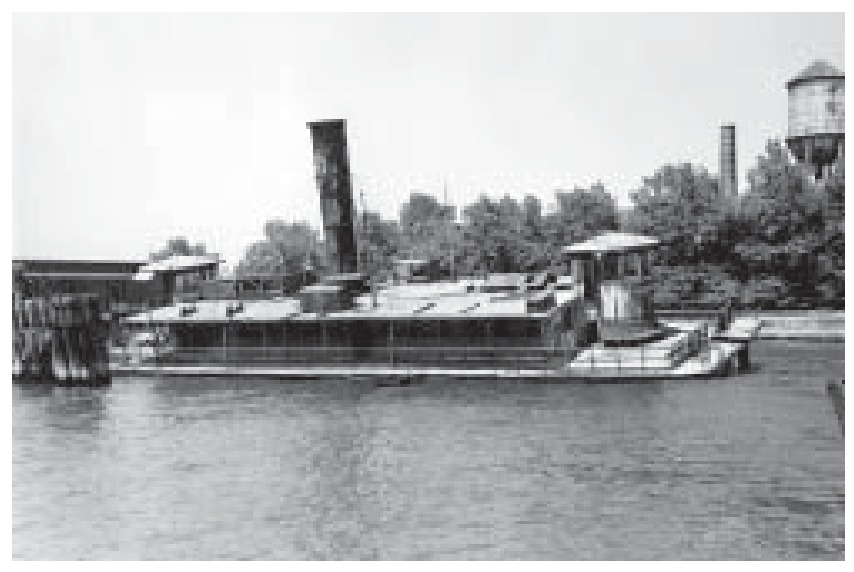

Figure 2: Ellis Island Ferry.

In a preliminary project, a smaller simulation has been completed of the Ellis Island Ferry (Figure 2), which carried immigrants to Manhattan from 1901 to 1953, and sank at her moorings in 1968 at Ellis Island. The SCRU wanted information about the stability of the wreck under several salvage scenarios. They used this information to recommend to the National Park Service not to raise the wreck. This project allowed NIST to work out the details of water and mud loadings on submerged hulls, which will be used in the model of the Arizona.

The tools developed in this project will be transferred to companies and organizations that are responsible for monitoring submerged wrecks containing hazardous materials, allowing them to better simulate critical conditions.

In addition to these two ships, we are providing assistance and technical expertise to NPS, NOAA, and the Navy regarding preservation of the CSS Hunley (on shore in Charleston, SC), the USS Monitor (partially salvaged), and NOAA's monitoring and scientific surveys of the wreck of the RMS Titanic, building on past work on this ship.

\section{Contributors and Collaborators}

L. Ma (Metallurgy Division, NIST); L. Murphy, M. Russell, D. Conlin (Submerged Cultural Resources Unit, National Park Service); Lt. J. Weirich (NOAA); Cdr. J. Morganthou (CINCPAC, U.S. Navy) 


\section{Materials Structure Characterization}

Fundamental to the field of materials science and engineering is the study of the relationships between processing, structure, properties, and performance of materials. Therefore, tools and techniques for the characterization of materials structure is a cornerstone of the field. The NIST Materials Science and Engineering Laboratory (MSEL) has a long tradition of supporting and developing measurement methods and facilities for materials structure characterization. Facilities within MSEL include optical and electron microscopy, optical and electron scattering and diffraction, a state-of-the-art x-ray diffractometer, the NIST Center for Neutron Research, and experimental stations at the National Synchrotron Light Source (NSLS) at Brookhaven Laboratory and at the Advanced Photon Source (APS) at Argonne National Laboratory. At the NSLS, NIST operates a soft $x$-ray station in partnership with Dow and Brookhaven National Laboratory. At the APS, NIST is a partner with the University of Illinois at Urbana/Champaign, Oak Ridge National Laboratory, and UOP in a collaboration called UNICAT. At both facilities, NIST scientists and researchers from industry, universities, and government laboratories perform state-of-the-art measurements on a wide range of advanced materials. NIST scientists have consistently advanced the limits of these facilities in order to improve spatial resolution and sensitivity needed, for example, to interrogate the microstructure of both highly anisotropic and/or gradient materials such as advanced thermal coatings and fuel cell systems. Studies currently underway at these facilities include: in-situ measurements of nanoparticle production; structure and dispersion of carbon nanotubes; three-dimensional imaging of natural and artificial tissues; surface and subsurface damage in UV lithography optics; strain-induced ferroelectric transitions in thin films; and determination of molecular orientation and bond concentration on chemically heterogeneous surfaces.

The materials characterization program has a strong emphasis on electron microscopy. The MSEL Electron Microscopy Facility provides structure and compositional characterization of a wide range of materials. The facility consists of two transmission electron microscopes (TEMs), three scanning electron microscopes (SEMs), a specimen preparation laboratory, and an image analysis/computational laboratory. The JEM3010 TEM resolves the atomic structure and employs an energy selecting imaging filter and $\mathrm{x}$-ray detector (EDS) for analytical characterization of thin foil specimens. The JSM6400 SEM employs electron backscattered diffraction/phase identification and EDS systems to characterize the texture and composition of materials. Highlights from the facility for FY2003 include: the computer domain is now active, providing streamlined user access and network file storage; a research collaboration with the NIST Semiconductor Electronics Division is underway to characterize quantum effects in confined Si devices; the size and shape of III-V quantum dots are characterized with the NIST Optoelectronics Division; and composition maps of electrodeposited nanowires with tunable magnetic properties are generated in collaboration with Johns Hopkins University.

This MSEL program also incorporates standards activities. A state-of-the-art x-ray diffractometer has been developed to study the metrology of powder diffraction in order to develop the next generation of diffraction standard reference materials. A variety of standard reference materials (SRMs) needed by the U.S. polymers industry, research laboratories, and other federal agencies have recently been developed: polyethylene of narrow mass distribution; nonlinear fluids for rheological measurements; melt flow standards; and the first reference biomaterial, an orthopedic grade ultra-high molecular weight polyethylene.

Recent program activities utilize matrix-assisted laser desorption/ionization time-of-flight mass spectrometry (MS) to address the need for improved characterization of the molecular structure of polyolefins, a dominant commercial polymer. This work has extended the upper mass limit detectable by MS to $15,000 \mathrm{~g} / \mathrm{mol}$ and, by observing individual oligomeric species by mass, has revealed details of the molecular structure.

A major cross-cutting activity within this program is the multi-scale, multi-modal imaging and visualization project. The goal of this project is to combine disparate sets of 3-D imaging data that contain complementary information on overlapping length scales to produce an interactive visualization scheme for multivariate data sets. This year focused on further improvements and additions to the suite of imaging tools for tissue engineering metrology. Osteoblasts cultured in a poly ( $\varepsilon$-caprolactone) scaffold have been imaged, demonstrating the advantages of optical coherence and confocal fluorescence microscopies over conventional laser scanning confocal microscopy. This instrument was upgraded by completely rebuilding the image acquisition software, hardware, and optical train. These improvements enabled an increase in image acquisition speed (2X) and accuracy (from $5 \mu \mathrm{m}$ to $360 \mathrm{~nm}$ ).

Contact: John E. Bonevich 


\section{Nanoscale Characterization by Electron Microscopy}

\begin{abstract}
Electron microscopy is used to characterize the structure and composition of materials at the nanometer scale to better understand and improve their properties. New measurement techniques in electron microscopy are being developed and applied to materials science research. The MSEL Electron Microscopy Facility serves the Metallurgy, Ceramics, and Polymers Divisions as well as other NIST staff and outside collaborative research efforts.
\end{abstract}

\section{John E. Bonevich}

Atomic-scale structure and compositional characterization of materials can lend crucial insights to the control of their properties. For instance, direct observation of local structures by transmission electron microscopy (TEM) provides an important information feedback to the optimization of crystal growth and processing techniques. Various characteristics may be observed such as crystal structure and orientation, grain size and morphology, defects, stacking faults, twins and grain boundaries, second phase particles - their structure, composition and internal defect structure, compositional variations as well as the atomic structure of surfaces, and interfaces.

The MSEL Electron Microscopy Facility consists of two transmission electron microscopes, three scanning electron microscopes, a specimen preparation laboratory, and an image analysis/computational laboratory. The JEM3010 TEM resolves the atomic structure and employs an energy selecting imaging filter (IF) and x-ray detector (EDS) for analytical characterization of thin foil specimens. The JSM6400 SEM employs electron backscattered diffraction/phase identification (EBSD) and EDS systems to characterize the texture and composition of materials.

Highlights from the EM Facility for FY2003 include:

- Facilities computer resources have been upgraded, providing streamlined user access and network file storage.

- Collaboration with the Semiconductor Electronics and Electricity Divisions (EEEL) to characterize quantum effects in confined Si devices.

- Characterization of the size and shape of III-V quantum dots, with the Optoelectronics Division (EEEL).

- Compositional mapping of electrodeposited nanowires with tunable magnetic properties, fabricated at Johns Hopkins University (Figure 1).
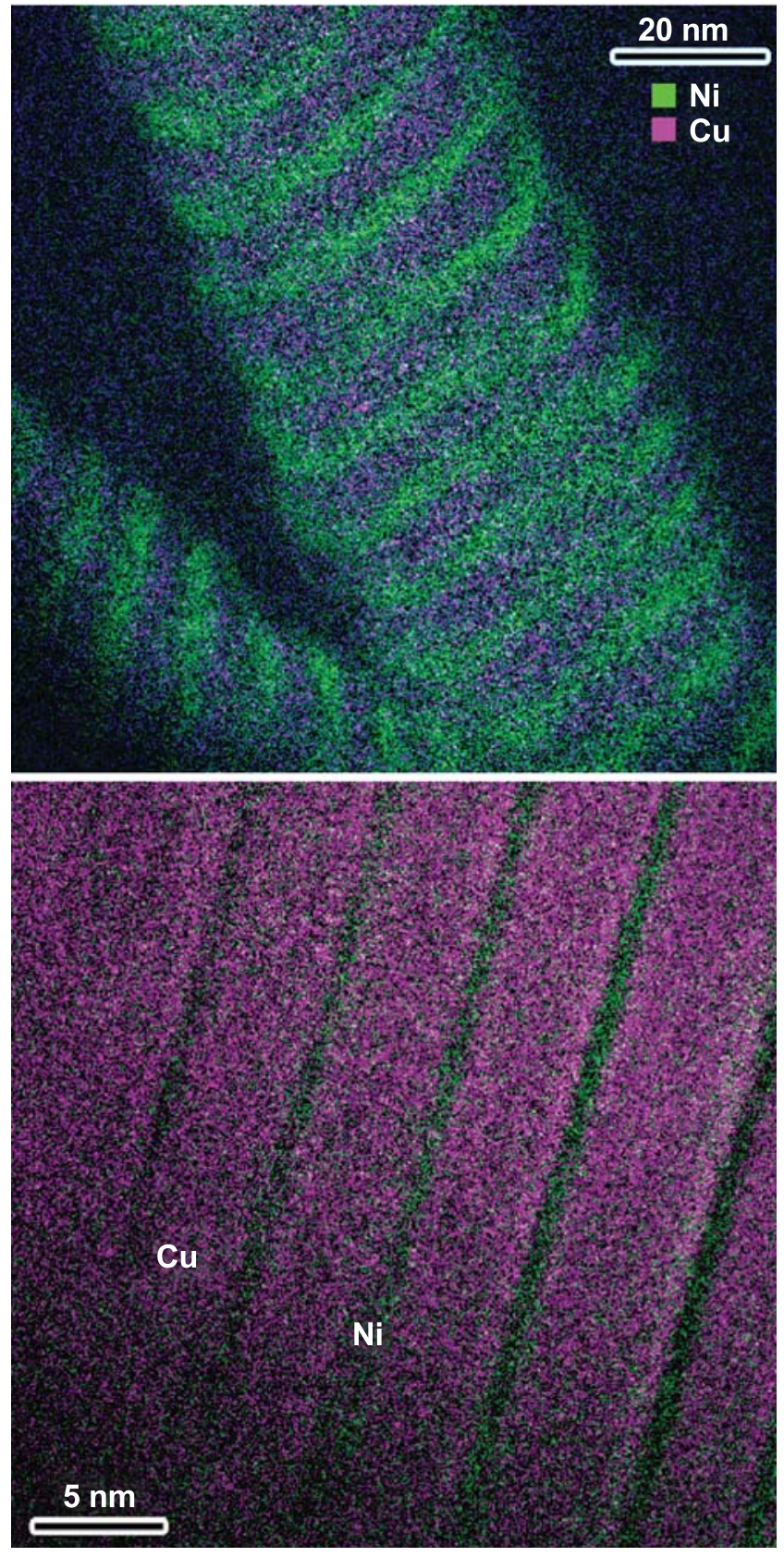

Figure 1: Composition maps of $\mathrm{Ni} / \mathrm{Cu}$ electrodeposited multilayer nanowires. Modulation of the composition wavelength provides a means to tune the magnetic properties as desired. The Ni layer thickness varies from $5 \mathrm{~nm}$ (top) to $1 \mathrm{~nm}$ (bottom).

\section{Contributors and Collaborators}

D. Josell, T. Moffat, R.D. McMichael (Metallurgy Division, NIST); A. Roshko, S.Y. Lehman (Optoelectronics Division, NIST); E. Vogel, N. Zimmerman (Semiconductor Electronics Division, NIST); P. Searson (Johns Hopkins University) 


\section{USAXS Imaging: Microscope Development and Validation}

\begin{abstract}
Modeling the behavior of complex materials requires detailed knowledge of the underlying three-dimensional microstructure. Ultra-smallangle X-ray scattering (USAXS) imaging is a new class of synchrotron $X$-ray imaging techniques that uses USAXS as the contrast mechanism. The technique provides imaging and statistical data that cannot be obtained using any other experimental methods.
\end{abstract}

\section{Lyle E. Levine, David J. Pitchure, and Gabrielle G. Long}

U SAXS imaging was developed by NIST researchers and was first demonstrated in May 2000. The primary advantages over existing X-ray imaging techniques are its inherently higher contrast and its USAXS-derived ability to provide quantitative data on object shapes and size distributions. USAXS imaging has broad applicability to a wide range of material systems including metals, ceramics, polymers, and biological materials. Over the past year, work has concentrated on two main areas. First, major improvements in the instrumentation have greatly improved the imaging capabilities, and second, a wide range of material systems have been explored to determine where USAXS imaging can have the greatest impact.

The USAXS imaging experiments are conducted on the UNICAT sector 33 insertion-device beamline at Argonne National Laboratory's Advanced Photon Source. A high-intensity, parallel, monochromatic $\mathrm{X}$-ray beam passes through entrance slits that define the size, shape and position of the beam. Imperfections in the X-ray beam are removed using multiple Bragg reflections within a $<111>$ channel-cut Si crystal referred to as the collimator. Within the sample, local density variations from the microstructure produce X-ray scattering at small angles. The X-rays leaving the sample are then angle-filtered using another pair of $<111>$ Si crystals referred to as the analyzer. The only X-rays from the sample that can pass through the analyzer are those scattered by the microstructure at a specific angle. This angle can be selected by rotating the analyzer. Images are then formed by either exposing nuclear emulsion plates or using an X-ray camera system.

During the past year, several major improvements have been made in the instrumentation. These include new analyzer crystals whose surfaces were chemically polished to minimize image aberrations, a multiple-sample motorized rotation stage for doing stereo imaging and a motorized rotating goniometer stage for aligning the samples and then rotating them over $180^{\circ}$ in situ. The most important improvement in instrumentation was the design, construction, and testing of a new high-resolution X-ray camera. In this device, the X-rays hit a single-crystal scintillator that converts them to visible light. The resulting image is then magnified using an optical microscope system using a cooled CCD chip, infinity-focus objective lenses, an auxiliary lens system to produce a real image on the CCD plane, and a mirror to shift the optical axis away from the X-ray beam. The spatial resolution of the camera is approximately $1 \mu \mathrm{m}$. Remote computer control is available for all operations of the USAXS imaging equipment, and sequences of images (such as during sample rotations) can be acquired automatically using macro programs.

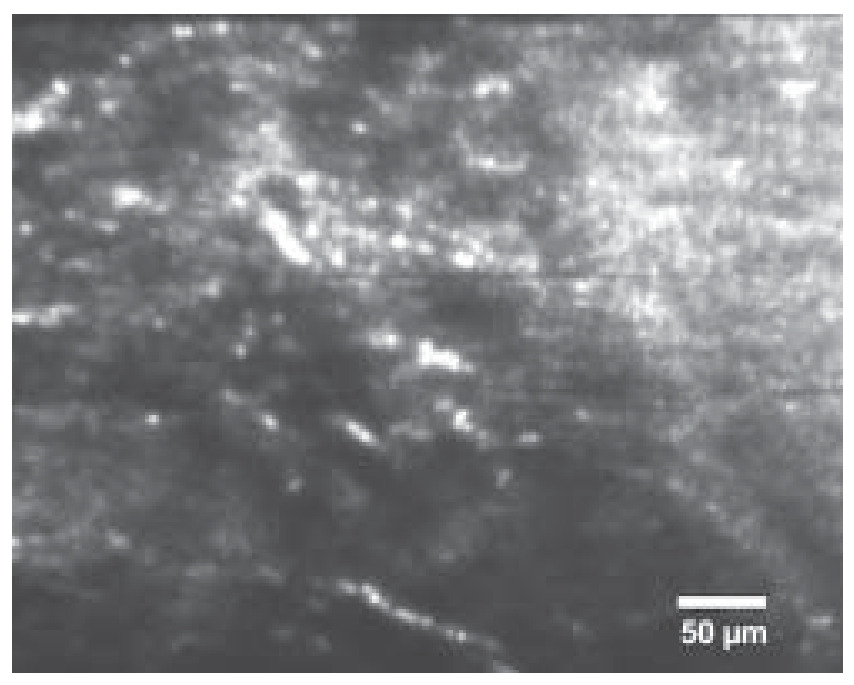

Figure 1: USAXS image of density variations within the Allende meteorite.

As examples of the technique's versatility, USAXS imaging has been successful at imaging creep cavities and cracks in copper, fatigue-generated voids in lead-free solder balls in microelectronic devices, silica beads embedded in polycarbonate and polypropylene, the Allende meteorite (see Figure 1), indentation crack surfaces in ceramics, artificial polymer tissue scaffolds, and fiber structures within bovine and human cartilage.

\section{Contributors and Collaborators}

J. Ilavsky (Ceramics Division, NIST); J. Dunkers (Polymers Division, NIST); P. Jemian (UNICAT); C. Muehleman (Rush Medical College); Denton Ebel (American Museum of Natural History) 



\section{Metallurgy Division FY03 Annual Report Publication List}

\section{Microstructural Origins of Surface Roughening and Strain Localization}

Banovic, S.W., and T. Foecke. "Evolution of strain-induced microstructure and texture in commercial aluminum sheet under balanced biaxial stretching." Metallurgial and Materials Transactions A, 34A (3), 657-671, 2003.

Banovic, S.W., M.D. Vaudin, T.H. Gnaeupel-Herold, K.P. Rodbell, and D. Saylor. "Studies of deformation induced texture development in sheet materials using diffraction techniques." Materials Science and Engineering A, (in press), 2003.

Shim, Y., M.R. Stoudt, L.E. Levine, S.W. Banovic, and R.J. Fields. "Material model for strain-induced surface roughening of sheet metal." Numisheet 2002: 5th International Conference and Workshop on Numerical Simulation of 3D Sheet Forming Processes. Ed. D.Y. Yang, et al. Seoul, Korea, pp. 155-160, 2002.

Stoudt, M.R., S.W. Banovic, and T. Quarrick. "Evolution of deformation induced surface morphologies developed on Fe-base sheet metals." Automotive Sheet Steels. Ed. B.J. Allen.

Warrendale, PA: TMS, 2003.

Stoudt, M.R., and R.E. Ricker. "The influence of grain size on the roughening behavior of Al-Mg alloys." Innovations in Processing and Manufacturing of Sheet Materials. Ed. M. Demeri. Warrendale, PA: TMS, pp. 237-244, 2001.

Stoudt, M.R., R.C. Cammarata, and R.E. Ricker. "Influence of nanometer-scale multilayered thin films on fatigue crack initiation." Thin Films - Stresses and Mechanical Properties VIII. Ed. R. Vinci, et. al. North Holland, NY: MRS, pp. 15-17, 1999.

Stoudt, M.R. "The influence of surface roughness on the deforming friction behavior of $\mathrm{Al}-\mathrm{Mg}$ alloys." Aluminum 2002. Ed. M.H. Skillingberg and S.K. Das. Warrendale, PA: TMS, pp. 27-34, 2002.

Stoudt, M.R., and R.E. Ricker. "The relationship between grain size and the surface roughening behavior of Al-Mg alloys." Metallurgical and Materials Transactions A, 33A (9), 2883-2889, 2002.

\section{Plasticity, Fabrication Processes, and Performance}

deWit, R. "Determination of the single crystal elastic constants by diffraction from a cubic polycrystal." NISTIR 6944, 2003.
Ma, L., T.F. Zahrah, and R.J. Fields. "Numerical 3D simulation of cold compaction and springback for particulate reinforced composites." Powder Metallurgy Journal, (in press), 2003.

\section{Underlying Processes of Plastic Deformation in Metal Alloys}

Delos-Reyes, M.A., M.E. Kassner, and L.E. Levine. "X-ray diffraction and the existence of long range internal stresses." Proceeding of Plasticity 2003, (in press), 2003.

Kramer, D.E., M.F. Savage, and L.E. Levine. "AFM observations of slip band development in $\mathrm{Al}$ single crystals." Acta Materialia, (submitted), 2003.

Levine, L.E., and R.M. Thomson. "A statistical connection between dislocations and mechanical properties." Dislocations, Plasticity and Metal Forming. Ed. A. Khan. Fulton: Neat Press, (in press), 2003.

Slutsker, J., and A. Roytburd. "Control of intrinsic instability of superelastic deformation." International Journal of Plasticity, 18, No. 11, 1561-1581, 2002.

\section{Atomization Processing - Recommended Practices}

Biancaniello, F.S., R.D. Jiggetts, M.R. Stoudt, R.E. Ricker, and S.D. Ridder. "Suitability of powder processed high nitrogen stainless steel alloys for high performance applications." 2002 Advanced in Powder Metallurgy and Particulate Materials, Part 3 Particulate Production, MPIF, 2002.

Mates, S.P., F.S. Biancaniello, and S.D Ridder. "An alternative view of close-coupled gas atomization of liquid metals." 2002 Advances in Powder Metallurgy and Particulate Materials, Part 3 - Particulate Production, MPIF, 2002.

Mates, S.P., and G.S. Settles. "A study of liquid metal atomization using close-coupled nozzles, part 1: gas dynamics." Atomization and Sprays, (in press), 2003.

Mates, S.P., and G.S. Settles. "A study of liquid metal atomization using close-coupled nozzles, part 2: atomization behavior." Atomization and Sprays, (in press), 2003.

Ridder, S.D. "Measurement and control of metal flow-rate in a gas-metal atomizer." 2002 Advances in Powder Metallurgy and Particulate Materials, Part 3 Particulate Production, MPIF, 2002. 


\section{High Speed Machining}

Basak, D., H.W. Yoon, R. Rhorer, T.J. Burns, and T. Matsumoto. "Temperature control of pulse heated specimens in a Kolsky bar apparatus using microsecond time-resolved pyrometry." International Journal of Thermophysics, (in press), 2003.

Basak, D., H.W. Yoon, R. Rhorer, and T.J. Burns. "Microsecond time-resolved pyrometry during rapid resistive heating of samples in a Kolsky bar apparatus." American Institute of Physics, (in press), 2003.

Rhorer, R., L. Levine, T. Burns, R. Fields, H. Yoon, M. Davies, D. Basak, E. Whitenton, G. Blessing, B. Dutterer, and M. Kennedy. "Constitutive model data for machining simulation using the NIST pulse-heated Kolsky bar." Dislocations, Plasticity and Metal Forming. Ed. A.S. Khan. Fulton: Neat Press, pp. 103-105, 2003.

Rhorer, R., D. Basak, G. Blessing, T. Burns, M. Davies, B. Dutterer, R. Fields, M. Kennedy, L. Levine, E. Whitenton, and H. Yoon. "Kolsky bar with electrical pulse heating of the sample." Proceedings for Society of Experimental Mechanics Annual Conference, Session No. 4, Paper No. 119, Society of Experimental Mechanics, 2003.

Yoon, H.W., D. Basak, R. Rhorer, E. Whitenton, T. Burns, R. Fields, and L. Levine. "Thermal imaging of metals in a Kolsky-bar apparatus." Thermosense XXV. Eds. E. Cramer and X.P. Maldague. Proceedings of SPIE, 5073, 284-294, 2003.

\section{Phase Field Modeling of the Electrochemical Interface}

Guyer, J.E., W.J. Boettinger, J.A. Warren, and G.B. McFadden. "Phase Field Modeling of Electrochemistry Equilibrium." Physical Review E, (submitted), 2003.

Guyer, J.E., W.J. Boettinger, J.A. Warren, and G.B. McFadden. "Phase Field Modeling of Electrochemistry: Kinetics." Physical Review E, (submitted), 2003.

\section{Combinatorial Methods for Thin Films}

Lu, C.J., L.A. Bendersky, K. Chang, and I. Takeuchi. "HRTEM study on the extended defect structure of epitaxial $\mathrm{Ba}_{0.3} \mathrm{Sr}_{0.7} \mathrm{TiO}_{3}$ thin films grown on (001) LaA103." Proceedings of MRS Symposium, (in press), 2003.

Lu, C.J., L.A. Bendersky, K. Chang, and I. Takeuchi. "High resolution electron microscopy study on the defect structure and strain relaxation of epitaxial $\mathrm{Ba}_{0.3} \mathrm{Sr}_{0.7} \mathrm{TiO}_{3}$ thin films grown on (001) LaA10." MRS Proceedings, Vol. 751, Z3.3, 2003.
Lu, C.J., L.A. Bendersky, K. Chang, and I. Takeuchi. "Dissociation and evolution of threading dislocations in epitaxial $\mathrm{Ba}_{0.3} \mathrm{Sr}_{0.7} \mathrm{TiO}_{3}$ thin films grown on (001) $\mathrm{LaAlO}_{3}$." Journal of Applied Physics, 93 (1), 1, 2003.

Lu, C.J., L.A. Bendersky, K. Chang, and I. Takeuchi. "High-resolution identifications of $1 / 2<110>$ stacking faults in epitaxial $\mathrm{Ba}_{0.3} \mathrm{Sr}_{0.7} \mathrm{TiO}_{3}$ thin films." Philosophical Magazine, 83, 1565, 2003.

\section{Phase Diagrams for III-V Thin Films and Metal Interconnects}

Bendersky, L.A., D. Tsvetkov, and Y. Melnik. "Transmission electron microscopy study of a defected zone in $\mathrm{GaN}$ on a $\mathrm{SiC}$ substrate grown by hydride vapor phase epitaxy." Journal of Applied Physics, 94, Issue 3, 1676-1685, 2003.

Davydov, A.V., and U.R. Kattner. "Revised Thermodynamic description of the Co-Mo system." Journal of Phase Equilibria, 24, 209, 2003.

Davydov, A.V., L.A. Bendersky, W.J. Boettinger, D. Josell, M.D. Vaudin, C.S. Chang, and I. Takeuchi. "Combinatorial investigation of structural quality of $\mathrm{Au} / \mathrm{Ni}$ contacts on GaN." Applied Surface Science Journal, (in press), 2003.

Lu, C.J., A. Davydov, D. Josell, and L.A. Bendersky. "Interfacial reactions of $\mathrm{Ti} / \mathrm{n}-\mathrm{GaN}$ contacts at elevated temperature." Journal of Applied Physic, 94, No. 1, 245-253, (in press), 2003.

Lu, C.J., and L.A. Bendersky. "Suppression of threading dislocations in $\mathrm{InN}$ thin films grown on (0001) sapphire with a GaN buffer layer." Applied Physics Letters, (in press), 2003.

Motayed, A., A.V. Davydov, L.A. Bendersky, M.C. Wood, M.A. Derenge, D.F. Wang, K.A. Jones, and S.N. Mohammad. "Novel high-transparency Ni/Au bilayer contacts to n-type GaN. Journal of Applied Physics, 92, 5218-5227, 2002.

Sanford, N.A., L.H. Robins, A.V. Davydov, A. Shapiro, A.V. Tsvetkov, A.V. Dmitriev, S. Keller, U.K. Mishra, and S.P. DenBars. "Measurements of the refractive indices of MOCVD and HVPE grown AlGaN films using prism-coupling techniques correlated with spectroscopic reflection/transmission analysis." Materials Research Society Symposium Proceedings, 743, L11.21.1-6, 2003.

Schenck, P., D.L. Kaiser, and A.V. Davydov. "High-throughput characterization of the optical properties of compositionally graded combinatorial films." Applied Surface Science Journal, (in press), 2003. 
Unland, J., B. Onderka, A. Davydov, and R. Schmid-Fetzer. "Thermodynamics and phase stability in the Ga-N system." Journal of Crystal Growth, 256, 33, 2003.

\section{Informatics and Visualization in Materials Data Delivery}

Davydov, A.V., and U.R. Kattner. "Revised thermodynamic description of the Co-Mo system." Journal of Phase Equilibria, 24, 209, 2003.

Kattner, U.R., J.E. Morral, and W.J. Boettinger. "Databases for computational thermodynamics and diffusion modeling." Journal of Phase Equilibria, (in press), 2003.

Kattner, U.R., W.J. Boettinger, and J.E. Morral. "Database for computational thermodynamics and diffusion modeling." Workshop Report, NISTIR 6927, 2003.

\section{Reaction Path Analysis in Multicomponent Systems}

Campbell, C.E., W.J. Boettinger, T. Hansen, P. Merewether, and B.A. Mueller. "Examination of multicomponent diffusion between two Ni-base superalloys." Properties of Complex Inorganic Solids 3. Ed. P. Turchi, et al. New York: Kluwer Academic Publishers, (in press), 2003.

Campbell, C.E., and U.R. Kattner. "Assessment of the Cr-B system and extrapolation to the Ni-Al-Cr-B system." CALPHAD, 26, No. 3, 477-490, 2003.

\section{Bridging Length Scales in Theory and Modeling: Microstructure Evolution and Material Mechanics}

Alikakos, N.D., P.W. Bates, J.W. Cahn, P.C. Fife, G. Fusco, and G.B. Tangoglu. "Extreme Anisotropy of Diffusion Interfaces in Crystals." SIAM Journal of Applied Mathematics, (in press), 2003.

Boettinger, W.J. "Phase field models for eutectic solidification." Journal of Metals, (submitted), 2003.

Boettinger, W.J., J.A. Warren, C. Beckermann, and A. Karma. "Simulation of solidification." Annual Review Materials Research, 32, 163-194, 2002.

Cahn, J.W., and J. Taylor. "A unified approach to motion of grain boundaries, relative tangential translation along grain boundaries, and grain rotation." Acta Materialia, (in press), 2003.
Foecke, T.J., and D.E. Kramer. "In situ TEM observations of fracture in nanolaminated metallic thin films." International Journal of Fracture, (in press), 2003.

Granasy, L., T. Pusztai, J.A. Warren, J.F. Douglas, T. Börzönyi, and V. Ferreiro. "Growth of "dizzy dendrites' in a random field of foreign particles." Nature Materials, 2, 92-96, 2003.

Lewis, D.J., W.J. Boettinger, and J.A. Warren. "Three dimensional phase field modeling of binary eutectics." Modeling of Casting, Welding and Advanced Solidification Processes, $X$. Ed. D.M. Stefanescu, J.A. Warren, M.R. Jolly, and J.M. Drane. Warrendale, PA: TMS, pp. 5-12, 2003.

Lewis, A.C., D. Josell, and T.P. Weihs. "Stability in thin film multilayers and microlaminates: the role of free energy, structure, and orientation at interfaces and grain boundaries." Scripta Materials, 48 (8), 1079-1085, 1 April 2003.

Lewis, D.J., T. Puztai, L. Granasy, J.A. Warren, and W.J. Boettinger. "Phase field models for eutectic solidification." JOM, (in press), 2003.

Sekerka, R.F., and J.W. Cahn. "Solid-liquid equilibrium for non-hydrostatic stress." Acta Materialia, (submitted), 2003.

Warren, J.A., R. Kobayashi, A.E. Lobkovsky, and W.C. Carter. "A phase field model of materials with crystalline interfaces." Acta Materialia, (in press), 2003.

Warren, J.A., L. Loginova, L. Granasy, T. Borzsonyi, and T.Pusztai. "Phase field modeling of alloy polycrystals." Proceedings for MCWASP Conference, (in press), 2003.

\section{Modeling Solidification, Electrodeposition, and Microstructure Evolution}

Granasy, L., T. Pusztai, T. Borzonyi, J.A. Warren, B. Kvamme, and P.F. James. "Nucleation and polycrystalline solidification in binary phase field theory." Physics and Chemistry of Glasses, (submitted), 2003.

\section{Electrochemical Processing of Nanostructure Materials}

Ford, A.X., J.E. Bonevich, R.D. McMichael, M.D. Vaudin, and T.P. Moffat. "Structure and magnetic properties of electrodeposited Co on n-GaAs(001)." Journal of Electrochemical Society, (in press), 2003. 


\section{Superconformal Film Growth: Measurement and Modeling}

Baker, B.C., M. Feeman, B. Melnick, D. Wheeler, D. Josell, and T.P. Moffat. "Superconformal electrodeposition of silver from a $\mathrm{KAg}(\mathrm{CN}) 2-\mathrm{KCN}$ KSeCN electrolyte." Journal of Electrochemical Society, 150, No. 2, C61-C66, 2003.

Baker, B.C., C. Witt, D. Josell, D. Wheeler, and T.P. Moffat. "Superconformal silver deposition using KSeCN derivitized substrates." Electrochemical and Solid State Letters, 6, No. 5, C67-C69, 2003.

Josell, D., B. Baker, and C. Witt. "Via filling by electrodeposition - superconformal silver and copper and conformal nickel." Journal of Electrochemical Society, 149, No. 12, C637-C641, December 2002.

Josell, D., S. Kim, D. Wheeler, T.P. Moffat, and S.G. Pyo. "Quantifying superconformal filling of the submicrometer features through surfactant catalyzed chemical vapor deposition." Proceedings of 2003 Spring Meeting of Electrochemical Society, (in press), 2003.

Josell, D., S. Kim, D. Wheeler, T.P. Moffat, and S.G. Pyo. "Interconnect fabrication by superconformal iodine-catalyzed chemical vapor deposition of copper." Journal of Electrochemical Society, 150, No. 5, C368-C373, 2003.

Josell, D., T.P. Moffat, and D. Wheeler. "An exact, algebraic solution for the incubation period of superfill." Electrochemical Society, (in press), 2003.

Josell, D., D. Wheeler, C. Witt, and T.P. Moffat. "Seedless superfill: copper electrodeposition in trenches with ruthenium barriers." Electrochemical and Solid State Letters, 6, No. 10, C143-C145, 2003.

McFadden, G.B, S.R. Coriell, T.P. Moffat, D. Josell, D. Wheeler, W. Schwarzacher, and J. Mallett. "A mechanism for brightening: linear stability analysis of the curvature enhanced accelerator coverage model." Journal of the Electrochemical Society, 150, No. 9, C591-C599, 2003.

Moffat, T.P., B.C. Baker, D. Wheeler, and D. Josell. "Accelerator aging effects during copper electrodeposition." Electrochemical and Solid State Letters, 6, No. 4, C59-C62, 2003.

Moffat, T.P., and D. Josell. "Electrodeposition of copper in the SPS-PEG-C1 additive system: I. Kinetic measurements: influence of SPS." Journal of Electrochemical Society, (in press), 2003.
Moffat, T.P., D. Wheeler, C. Witt, and D. Josell. "Superconformal electrodeposition using derivitized substrates." Electrochemical Solid State, 5, No. 12, C110-C112, December, 2002.

Pyo, S.G., S. Kim, D. Wheeler, T.P. Moffat, and D. Josell. "Seam-free fabrication of submicrometer copper interconnects by iodine-catalyzed chemical vapor deposition." Journal of Applied Physics, 93, No. 2, 1257-1261, January 15, 2003.

Wheeler, D., D. Josell, and T.P. Moffat. "Modeling superconformal electrodeposition using the level set method." Journal of the Electrochemical Society, 150, No. 5, C302-C310, 2003.

\section{Pb-free Surface Finishes: Sn Whisker Growth}

Bogustavsky, I., P. Bush, E. Kam-Lum, M. Kwoka, J. McCullen, K. Spalding, K. Vo, and M.E. Williams. "NEMI tin whisker test method standards." SMTAI Proceedings, (submitted), 2003.

Moon, K., C.E. Johnson, M.E. Williams, O. Kongstein, G.R. Stafford, C.A. Handwerker, and W.J. Boettinger. "Observed correlation of Sn oxide film to Sn whisker growth in $\mathrm{Sn}-\mathrm{Cu}$ electrodeposit for Pb-free Solders." Journal of Electronic Materials, (in press), 2003.

\section{Pb-free Solders and Solderability}

Bertocci, U. "An EQMB examination of $\mathrm{Cu}$ surface oxides in borate buffer." Electrochemica Acta, (submitted), 2003.

Boettinger, W.J., M.D. Vaudin, M.E. Williams, L.A. Bendersky, and W.R. Wagner. "EBSD and EDS study of the phase $\mathrm{NiSn}_{4}$." Journal of Electronic Materials, 32, 511-515, 2003.

Bradley, E., C.A. Handwerker, and J. Sohn. "Lead-free solder alloys: NEMI recommendation." Circuits Assembly, (in press), 2003.

Kattner, U.R. "Phase diagrams for solder alloys." Journal of Materials, 54 (12), 45-51, 2002.

Schaefer, R.J., and D.J. Lewis. "Directional solidification in a $\mathrm{AgCuSn}$ eutectic alloy." Journal of Electronic Materials, (in press), 2003.

\section{Solder and Solderability Measurements for Microelectronics}

Handwerker, C.A., J. Bath, E. Benedetto, E. Bradley, R. Gedney, T.A. Siewert, P. Snugovsky, and J. Sohn. "NEMI Lead-Free Assembly Project: comparison between $\mathrm{PbSn}$ and $\mathrm{SnAgCu}$ reliability and microstructures." SMTAI Proceedings, (in press), 2003. 


\section{Magnetic Properties and Standard Reference Materials}

Rao S., L.H. Bennett, E. Della Torre, A.P. Chen, and R.A. Fry. "Fluctuating field calculation for a bimodal medium." Journal of Applied Physics, 93, No. 10, 7798-7800, 2003.

\section{Magnetic Properties of Nanostructured Materials}

Kabanov, Y.P., V.S. Gornakov, V.I. Nikitenko, A.J. Shapiro, and R.D. Shull. "Nucleation and evolution of hybrid spin spiral in soft/hard ferromagnetic bilayer." Journal of Applied Physics, 93, 8244, 2003.

King, T.T., B.A. Rowlett, R.A. Ramirez, P.J. Sirron, E.R. Canavan, M.J. DiPirro, J.S. Panek, J.G. Tuttle, R.D. Shull, and R.A. Fry. "Rare-earth garnets and perovskites for space-based ADR cooling at high T and low H." AIP Conference Proceedings, 613, 1191, 2002.

Nikitenko, V.I., V.S. Gornakov, Y.P. Kabanov, A.J. Shaper, R.D. Shull, C.L. Chien, J.S. Jiang, and S.D. Bader. "Magneto-optic indicator film study of the hybrid exchange spring formation and evolution processes." Journal of Magnetism and Magnetic Materials, 19, 258-259, 2003.

Provenzano, V., J. Li, T.T. King, E.R. Canavan, P. Shirron, M.J. DiPorrp, and R.D. Shull. "Enhanced magnetocaloric effects in $\mathrm{R}_{3}\left(\mathrm{Ga}_{1-\mathrm{x}} \mathrm{Fe}_{\mathrm{x}}\right)_{5} \mathrm{O}_{12}(\mathrm{R}=\mathrm{Gd}$, Dy, Ho); $(\mathrm{O}<\mathrm{x}<1)$ nanocomposites." Journal of Magnetism and Magnetic Materials, (in press), 2003.

Shir, F., L. Yanik, L.H. Bennett, E. Della Torre, and R.D. Shull. "Room temperature active regenerative magnetic refrigeration: magnetic nanocomposites." Journal of Applied Physics, 93, 8295, 2003.

Shull, R.D., A.J. Shapiro, V.S. Gornakov, V.I. Nikitenko, and H.W. Zhao. "Stationary antiferromagnetic domains during magnetization reversal in an exchange-biased $\mathrm{FeMn} / \mathrm{Fe}_{76} \mathrm{Mn}_{6} \mathrm{C}_{18}$ bilayer." Journal of Applied Physics, 93, 8603, 2003.

Taketomi, S. and R.D. Shull. "Experimental verification of interactions between randomly distributed fine magnetic particles." Journal of Magnetism and Magnetic Materials, (in press), 2003.

Taketomi, S., A.J. Shaper, and R.D. Shull. "Structural effects on the magnetic character of yttrium-iron-garnet nanoparticles dispersed in glass composites." Journal of Applied Physics, 93, 7199, 2003.
Zheng, L.A., E.V. Barrera, and R.D. Shull. "Magnetic properties of the $\mathrm{Co}-\mathrm{C}(\mathrm{d} 60)$ and $\mathrm{Fe}-\mathrm{C}(\mathrm{d} 60)$ nanocrystalline magnetic thin films." Journal of Applied Physics, (submitted), 2003.

\section{Nanomagnetodynamics}

Della Torre, E., and L.H. Bennett. "Thermal longevity of nano-multilayer magnetic media." Proceedings of the 2002 2nd IEEE Conference on Nanotechnology, Piscataway, New Jersey, pp. 71-74, 2003.

Kunz, A., and R.D. McMichael. "Normal mode mixing and ferromagnetic resonance linewidth." IEEE Transactions of Magnetics, 38, No. 5, 2400-2402, (in press), 2002.

McMichael, R.D. "A classical model of extrinsic ferromagnetic resonance linewidth in ultra-thin films." IEEE Transactions on Magnetics, (submitted), 2003.

McMichael, R.D., A. Kunz, and D.J. Twisselmann. "Localized ferromagnetic resonance in inhomogeneous thin films." Physical Review Letters, Vol. 90, Art. No. 227601, 2003.

\section{New Materials for High Density Data Storage}

Bae, S., J.H. Judy, P.J. Chen, and W.F. Egelhoff, Jr. "Dependence of physical properties and giant magnetoresistance ratio on substrate position during rf sputtering of $\mathrm{NiO}$ and alpha- $\mathrm{Fe}_{2} \mathrm{O}_{3}$ for bottom spin valves." Applied Physics Letters, 81, 2208, 2002.

Bae, S., J.H. Judy, P.J. Chen, L. Gan, W.F. Egelhoff, Jr., J. Hautala, and D.B. Fenner. "Effects of Ar gas cluster ion beam (GCIB) processing on surface roughness, crystalline orientation texture, magnetic, and giant magnetoresistance (GMR) properties of reactively RF sputtered, alpha- $\mathrm{Fe}_{2} \mathrm{O}_{3}$ bottom GMR spin valves." IEEE Transactions of Magnetics, (in press), 2003.

Bae, S., P.J. Chen, W.F. Egelhoff, Jr., and J.H. Judy. "Effects of sputtering angle on surface roughness, chemical composition, and GMR behavior of $\mathrm{NiO}$ and alpha- $\mathrm{Fe}_{2} \mathrm{O}_{3}$ bottom spin-valves." IEEE Transactions of Magnetics, (in press), 2003.

Buchanan, J.D.R., T.P.A. Hase, B.K. Tanner, P.J. Chen, L. Gan, C.J. Powell, and W.F. Egelhoff, Jr. "Anomalously large intermixing in aluminum-transition metal bilayers." Physical Review B, Vol. 66, Art. No. 104427, 2002. 
Buchanan, J.D.R., T.P.A. Hase, B.K. Tanner, P.J. Chen, L. Gan, C.J. Powell, and W.F. Egelhoff, Jr. "Intermixing of aluminum-magnetic transition metal bilayers." Journal of Applied Physics, 93, 8044, 2003.

Carrey, J., A.E. Berkowitz, W.F. Egelhoff, Jr., and D. Smith. "Influence of interface alloying on the magnetic properties of $\mathrm{Co} / \mathrm{Pd}$ multilayers." Applied Physics Letters, (submitted), 2003.

Chopra, H.D., D.X. Yang, P.J. Chen, and W.F. Egelhoff, Jr. "Domain structure and magnetoelastic properties of $\mathrm{Fe} / \mathrm{Pd}$ ferromagnetic thin films and multilayers." Journal of Applied Physics, (in press), 2003.

Gan, L., R.D. Gomez, A. Castillo, P.J. Chen, C.J. Powell, and W.F. Egelhoff, Jr. "Ultra-thin aluminum oxide as a thermal oxidation barrier on metal films." Thin Solid Films, 415, 219, 2002.

Gan, L., R.D. Gomez, C.J. Powell, R.D. McMichael, P.J. Chen, and W.F. Egelhoff, Jr. "Thin $\mathrm{Al}, \mathrm{Au}, \mathrm{Cu}$, $\mathrm{Ni}, \mathrm{Ta}$, and $\mathrm{Fe}$ films as oxidation barriers for the Co surface in air." Journal of Applied Physics, 93, 8731, 2003.

Gan, L., R.D. McMichael, P.J. Chen, W.F. Egelhoff, Jr., and G.C. Lee. "Ultra-thin aluminum oxide as a thermal oxidation barrier on metal films." Thin Solid Films, 414, 219, 2002.

Chung, S.H., N. Garcia, W.F. Egelhoff, Jr., and R.D. Gomez. "Universal behavior of ballistic magnetoresistance versus scaled conductance in atomic nanocontacts." Physical Review Letters, Vol. 89, Art. No. 287203, 2002.

Chung, S.H., N. Garcia, W.F. Egelhoff, Jr., and R.D. Gomez. "Universal scaling of magnetoconductance in magnetic nanocontacts." Journal of Applied Physics, 93, 7939, 2003.

Gornakov, V.S., V.I. Nikitenko, W.F. Egelhoff, Jr., R.D. McMichael, A.J. Shapiro, and R.D. Shull.

"Ru spacer thickness dependences of the domain nucleation and growth in $\mathrm{Co} / \mathrm{Ru} / \mathrm{Co}$ synthetic antiferromagnet." Journal of Magnetism and Magnetic Materials, 258, 345 (2003).

Huang, H., K. Seu, W.F. Egelhoff, Jr., and A. Reilly. "Ultrafast pump-probe laser spectroscopy of the half-metal $\mathrm{CrO}_{2}$." Journal of Applied Physics, (in press), 2003.

Lee, C.G., J.-G. Jung, V.S. Gornakov, R.D. McMichael, A. Chen, and W.F. Egelhoff, Jr. "Effects of annealing on the GMR and domain structure stabilization in a $\mathrm{Py} / \mathrm{Cu} / \mathrm{Py} / \mathrm{MnIr}$ spin valve." Journal of Magnetism and Magnetic Materials, (in press), 2003.
Mallett, J.J., E.B. Svedberg, H. Ettedgui, T.P. Moffat, and W.F. Egelhoff, Jr. "A search for ballistic magnetoresistance (BMR) in electrodeposited Ni nanocontacts: feedback control used to select the contact resistance." Applied Physical Letters, (in press), 2003.

Osofsky, M.S., R.J. Soulen, Jr., G. Woods, and W.F. Egelhoff, Jr. "Enhanced $\mathrm{T}_{\mathrm{c}}$ near the metal/insulator transition: new insight into novel superconducting Materials." Physical Review Letters, (in press), 2003.

Rezende, S.M, A. Azevedo, F.M. de Aguiar, J.R. Fermin, W.F. Egelhoff, Jr., and S.S.P. Parkin. "Three-layer model for exchange anisotropy." Physical Review B, Vol. 66, Art. No. 064109, 2002.

Sanz, M., A. Papageorgopoulos, W.F. Egelhoff, Jr., M. Nieto-Vesperinas, and N. Garcia. "Wedge-shaped absorbing samples look left handed: the problem of interpreting negative refraction, and its solution," Physical Review B, (in press), 2003.

Seu, K., H. Huang, J.L. Showman, H. Showman, W.F. Egelhoff, Jr., and A. Reilly. "Co layer thickness dependence of exchange biasing of $\mathrm{IrMn} / \mathrm{Co}$ and FeMn/Co." Journal of Applied Physics, 93, 6611, 2003.

Siu, I.L., W.F. Egelhoff, Jr., H.D. Chopra, and D.X. Wang. "Magnetization reversal in epitaxial $\mathrm{CrO}_{2}$ films." Journal of Applied Physics, 92, 5409, 2002.

Svedberg, E.B., J.J. Mallett, H. Ettedgui, L. Gan, P.J. Chen, A.J. Shapiro, T.P. Moffat, and W.F. Egelhoff, Jr. "Possible ballistic magnetoresistancelike artifacts in electrodeposited nanocontacts." Journal of Applied Physics, (in press), 2003.

Torok, E.J., S. Zurn, S. Bae, P.J. Chen, W.F. Egelhoff, Jr., L.E. Sheppard, R. Spitzer, and J.H. Judy. "Transpinnor: a new giant magnetoresistive spin-valve device." Applied Physics, 91, 8414, 2002.

Vaz, C.F.A., E. Blackburn, M. Klaui, J.A.C. Bland, W.F. Egelhoff, Jr., E. Cambril, G. Faini, and W. Wernsdorfer. "Magnetoresistance magnetometry of $\left(\mathrm{Ni}_{80} \mathrm{Fe}_{20}\right)_{1-\mathrm{x}} \mathrm{Ir}_{\mathrm{x}}$ wires with varying anisotropic magnetoresistance ratio." Journal of Applied Physics, 93, 8104, 2003.

Woods, G.T., R.J. Soulen, Jr., I.I. Mazin, B. Nadgorny, M.S. Osofsky, J. Sanders, H. Srikanth, W.F. Egelhoff, Jr., and R. Datla. "Analysis of point-contact Andreev reflection spectra in spin polarization measurements." Physical Review B, (in press), 2003. 
Yang, D.X., E.J. Repetski, H.D. Chopra, B.J. Spencer, D.C. Parks, P.J. Chen, and W.F. Egelhoff, Jr. "A pinhole coupling strength in giant magnetoresistance spin valves: a statistical approach." Physical Review B, (in press), 2003.

Yang, D.X., E.J. Repetski, H.D. Chopra, P.J. Chen, and W.F. Egelhoff, Jr. "Highly deleterious role of small amounts of carbon in the giant magnetoresistance effect." Journal of Applied Physics, 93, 8415, 2003.

\section{Hardness Standardization - Rockwell, Vickers, Knoop}

Barbato, G., A. Germak, K. Herrmann, and S.R. Low. "Evolutions in hardness scales definition." Proceedings of XVII IMEKO World Congress, Dubrovnik, Croatia, June 22-27, pp. 978-981, 2003.

Barbato, G., A. Germak, and S.R. Low. "Proposal for a practical procedure for the expression of uncertainty in hardness measurement." Proceedings of XVII IMEKO World Congress, Dubrovnik, Croatia, June 22-27, pp. 986-991, 2003.

Jiang, J.S., S.D. Bader, H. Kaper, G.K. Leaf, R.D. Shull, A.J. Shapiro, V.S. Gornakov, V.I. Nikitenko, C.L. Platt, A.E. Berkowitz, S. David, and E.E. Fullerton. "Rotational hysteresis of exchange-spring magnets." Journal of Physics, D35, 2339, 2002.

Low, S.R., and J. Fink. "Effects of bending in brass Rockwell B scale test blocks." Proceedings of XVII IMEKO World Congress, Dubrovnik, Croatia, June 22-27, pp. 1030-1033, 2003.

Ma, L., S.R. Low, and J. Song. "Determining mechanical properties of $\mathrm{O} 1$ tool steel from reverse computation of indentation measurement." Proceedings of XVII IMEKO World Congress, Dubrovnik, Croatia, June 22-27, pp. 1005-1008, 2003.

Ma, L., S.R. Low, and J. Song. "FEA modeling and experimental comparisons of deformable ball indenters; effect on Rockwell B Hardness (HRB) tests." Journal of Testing and Evaluation, Vol. 31 (6), (in press), November 2003.

Ma, L., J. Zhou, A. Lau, S. Low, and R. deWit. "Self-similarity simplification approaches for the modeling and analysis of Rockwell Hardness indentation." Journal of Research of the National Institute of Standards and Technology, 107 (5), 401-412, September-October 2002.

Ma, L., S. Low, J. Zhou, J., J. Song, and R. deWit. "Simulation and prediction of hardness performance of Rockwell diamond indenters using finite element analysis." Journal of Testing and Evaluation, 30 (4), 265-273, July 2002.

\section{Nanoscale Characterization by Electron Microscopy}

Chen, M., L. Sun, J.E. Bonevich, D.H. Reich, C.L. Chien, and P.C. Searson. "Tuning the magnetic properties of multilayer nanowires." Applied Physics Letters, 82, 3310, 2003.

Chong, Y., P.D. Dresselhaus, S.P. Benz, and J.E. Bonevich. "Effects of interlayer electrode thickness in $\mathrm{Nb} /\left(\mathrm{MoSi}_{2} / \mathrm{Nb}\right) \mathrm{N}$ stacked Josephson junctions." Applied Physics Letters, 82, 2467, 2003.

Kramer, D.E., M.F. Savage, A. Lin, and T.J. Foecke. "Novel method for TEM characterization of deformation under nanoindents in nanolayered materials." Scripta Materialia, (submitted), 2003.

Lehman, S.Y., A. Roshko, R.P. Mirin, and J.E. Bonevich. "Investigation of the shape of InGaAs/GaAs quantum dots." Proceedings of Materials Research Society, Vol. 737, (in press), 2003.

Shin, K., H. Wang, S.K. Satija, C.C. Han, D. Josell, and J.E. Bonevich. "Rapid deformation of thin gold layers in polymer matrices studied by X-ray reflectivity." Journal of Applied Physics, 94 (3), 2115-2122, August 1, 2003.

\section{Other}

Basak, D., R.A. Overfelt, and D. Wang. "Measurement of specific heat capacity and electrical resistivity of industrial alloys using pulse heating techniques." International Journal of Thermophysics, (in press), 2003.

Bobordis, K., A. Seifter, A.W. Obst, and D. Basak. "Radiance temperatures and normal spectral emittances (in the wavelength range of $1500 \mathrm{~nm}$ to $5000 \mathrm{~nm}$ ) of tin, zinc, aluminum, and silver at their melting points by a pulse-heating technique." International Journal of Thermophysics, (in press), 2003.

Bobordis, K., A. Seifter, A.W. Obst, and D. Basak. "Validation of a new high-speed fiber-coupled four-wavelength infrared pyrometer in the range of $505 \mathrm{k}$ to $1234 \mathrm{k}$ by a pulse-heating technique, using the melting points of tin, zinc, aluminum, and silver as reference points." International Journal of Thermophysics, (in press), 2003.

Boettinger, W.J., C.E. Campbell, T. Hansen, P. Merewether, and B.A. Mueller. "Properties of complex inorganic solids." Ed. P.E.A. Turchi, A. Gonis, A. Meike, and K. Ragan. New York: Kluwer Academics/Plenum Publishers, (in press), 2003. 
Gayle, F.W. "FY2002 programs and accomplishments: MSEL materials for micro- and optoelectronics." NISTIR 6971, 2003.

Hooper, J.J., T.J. Foecke, L. Graham, and T.P. Weihs. "Metallurgical analysis of wrought iron from the RMS titanic." Marine Technology, (in press), 2003.

Kass, M., C.R. Brooks, D. Falcon, and D. Basak. "The formation of defects in Fe-Al alloys: electrical resistivity and specific heat measurements."

Intermetallics, 10, 951-966, 2002.

Kramer, D.E., and T. Foecke. "Observations of deformation and fracture in metallic nanolaminates." Philosophical Magazine A, 82, No. 17-18, 3375-3381, 2002.

Park, J.-K., D.-Y. Kim, H.-Y. Lee, J.E. Blendell, and C.A. Handwerker. "Crystallographic orientation dependent dissolution behavior of sapphire in anorthite liquid containing $\mathrm{Cr}_{2} \mathrm{O}_{3}$." Journal of the American Ceramic Society, 86 (6), 1014-1018, 2003.

Ricker, R.E. "Modeling the influence of crack path deviations on the propagation of stress corrosion cracks." Hydrogen Effects on Materials Behavior and Corrosion Deformation Interactions. Ed. N.R. Moody, A.W. Thompson, R.E. Ricker, G.S. Was, and R.H. Jones. Warrendale, PA: TMS, (in press), 2003.

Ricker, R.E., and A.K. Vasudevan. "The influence of grain boundary precipitation on the stress corrosion cracking of Al-Li and Al-Li-Cu alloys." Hydrogen Effects on Materials Behavior and Corrosion Deformation Interactions. Ed. N.R. Moody, A.W. Thompson, R.E. Ricker, G.S. Was, and R.H. Jones. Warrendale, PA: TMS, (in press), 2003.
Stafford, G.R., T. Tsuda, and C.L. Hussey. "Order/disorder in electrodeposited aluminum-titanium alloys." Journal of Mining and Metallurgy, 39, Issue 1-2B, 23-42, 2003.

Song, J., L. Ma, E. Whitenton, and T. Vorburger. "Bullet signature measurement at NIST." Proceedings of Measurement Science Conference, Anaheim, California, January 2003.

Song, J., T. Voburger, R. Clary, E. Whitenton, L. Ma, and S. Ballou. "Standards for bullets and casings." Materials Today, pp. 26-31, November 2002.

Stoudt, M.R., and R.E. Ricker. "Evaluating corrosion fatigue behavior through innovative electrochemical analyses." Metallurgical and Materials Transactions A, (in press), 2003.

Tsuda, T., C.L. Hussey, G.R. Stafford, and J.E. Bonevich. "Electrochemistry of titanium and the electrodeposition of Al-Ti alloys in the Lewis acid aluminum chloride-1 ethyl-3-methylimidazolium chloride melt." Journal of Electrochemical Society, 150, C234, 2003.

William, M.E., W.J. Boettinger, and U.R. Kattner. "Contribution to the $\mathrm{Zn}$-rich part of the $\mathrm{Zn}-\mathrm{Zr}$ phase diagram." Journal of Phase Equilibria, (submitted), 2003 . 


\section{Metallurgy Division}

Chief

Carol A. Handwerker

Phone: 301-975-6158

E-mail: carol.handwerker@nist.gov

\section{Deputy Chief}

Frank W. Gayle

Phone: 301-975-6161

E-mail: frank.gayle@nist.gov

\section{NIST Fellows}

William J. Boettinger

Phone: 301-975-6160

E-mail: william.boettinger@nist.gov

John W. Cahn

Phone: 301-975-5664

E-mail: john.cahn@nist.gov

\section{Group Leaders}

Electrochemical Processing Group

Gery R. Stafford

Phone: 301-975-6412

E-mail: gery.stafford@nist.gov

Magnetic Materials Group

Robert D. Shull

Phone: 301-975-6035

E-mail: robert.shull@nist.gov

Materials Performance Group

Stephen D. Ridder

Phone: 301-975-6175

E-mail: stephen.ridder@nist.gov

Materials Structure and Characterization Group Frank W. Gayle

Phone: 301-975-6161

E-mail: frank.gayle@nist.gov

Metallurgical Processing Group

Stephen D. Ridder

Phone: 301-975-6175

E-mail: stephen.ridder@nist.gov 


\section{Research Staff}

Banovic, Stephen W.

stephen.banovic@nost.gov

Metal forming

Mechanical properties

Texture analysis

\section{Beauchamp, Carlos R.}

carlos.beauchamp@nist.gov

Compositionally modulated alloys

Standard reference materials

\section{Baker, Brett C.}

brett.baker@nist.gov

Deposition of metals/alloys

\section{Bendersky, Leonid A.}

leonid.bendersky@nist.gov

Functional ceramics

Analytical and high-resolution TEM

High-temperature intermetallics

Combinatorial methods

Biancaniello, Frank S.

frank.biancaniello@nist.gov

Spray deposition measurements and diagnostics Inert gas atomization: metal powder

measurements and consolidation

Nitrogenated steels, standard reference materials

Special alloys, bulk metallic glasses

Biomaterials

\section{Boettinger, William J.}

william.boettinger@nist.gov

Relation of alloy microstructures to processing conditions; solidification

Thermal analysis

Solder

\section{Bonevich, John E.}

john.bonevich@nist.gov

Electron holography

Interfacial structure and chemistry

High resolution/analytical electron microscopy

Magnetic materials

\section{Campbell, Carelyn E.}

carelyn.campbell@nist.gov

Transient liquid phase bonding

Multicomponent diffusions simulations

Alloy design methodology

\section{Claggett, Sandra W.}

sandra.claggett@nist.gov

Specimen preparation for electron microscopy

Digital imaging

Specimen preparation for scanning electron microscopy

Image analysis

Metallography of materials

Specimen preparation for transmission of electron microscopy

\section{Drew, Rosetta V.}

rosetta.drew@nist.gov

Magnetometry

Database maintenance

Egelhoff Jr., William F. william.egelhoff@nist.gov

Giant magnetoresistance

Molecular beam epitaxy

Surface physics

Ultrahigh density data storage

Fields, Richard J.

richard.fields@nist.gov

Mechanical properties

Mechanical testing

Powder consolidation

Metal forming

Fink, James L.

james.fink@nist.gov

Hardness standards

Hardness testing

Mechanical properties measurement

Metal powder consolidation

Environmental corrosion

Metallography

Foecke, Timothy J.

timothy.foecke@nist.gov

Metal forming standards

Nanostructured materials

Experimental fracture physics

SEM, TEM, SPM

Micro- and nano-mechanics of materials

Dislocation-based deformation mechanisms

Historical metallurgy and failure analysis

\section{Gates, Hilary G.}

hilary.gates@nist.gov

Standard reference materials 


\section{Gayle, Frank W.}

frank.gayle@nist.gov

Structure/property relationships

Transmission electron microscopy

Aluminum metallurgy

Solder science

Guyer, Jonathan E.

jonathan.guyer@nist.gov

III-V semiconductors

Molecular beam epitaxy

Alloy film deposition

Stress-induced mass transport

\section{Handwerker, Carol A.}

carol.handwerker@nist.gov

Interface thermodynamics and kinetics

Solder science

Microstructure evolution

\section{Jiggetts, Rodney D.}

rodney.jiggetts@nist.gov

Substrate analysis

Grit blasting

Roughness measurements

Metallography of coatings

Image analysis

Micro analysis/characterization

Hot isostatic processing

Johnson, Christian E.

christian.johnson@nist.gov

Ultra-black coatings

Electroless deposition processes

Metallic glass alloy deposition

Microhardness SRM research

Chromium deposition

Pulse alloy deposition

Lead-free solder

\section{Josell, Daniel}

daniel.josell@nist.gov

Mechanical and thermal properties of multi-layer materials

Measurement and modeling of solder joint geometries

Filling of sub- $0.1 \mathrm{~mm}$ features by electrodeposition

\section{Kattner, Ursula R.}

ursula.kattner@nist.gov

Computational thermodynamics

Alloy phase equilibria evaluations

Solder alloy systems

Superalloy systems
Kelley, David R.

david.kelley@nist.gov

Microhardness SRM development

Dye penetration SRM development

Precious metal electrodeposition

Plating on aluminum

Electroplating

Levine, Lyle E.

lyle.levine@nist.gov

Diffraction theory

Dislocation-based deformation

Synchrotron x-ray techniques

Percolation theory

TEM, SEM, SPM

Lewis, Daniel J.

daniel.lewis@nist.gov

Modeling of eutectic solidification

Electric packaging

Low, II, Samuel R.

samuel.low@nist.gov

Hardness standards

Hardness testing

Mechanical properties of materials

Mechanical testing of materials

Luecke, William E.

william.luecke@nist.gov

High temperature mechanical testing

Creep of ceramics

Silicon nitride

Mates, Steven P.

steven.mates@nist.gov

Compressible fluid flow

Metal sprays

Fluid flow visualization

McMichael, Robert D.

robert.mcmichael@nist.gov

Giant magnetoresistance

Micromagnetic modeling

Ferromagnetic resonance

Nanocomposites

Moffat, Thomas P.

thomas.moffat@nist.gov

Electrochemistry

Scanning probe microscopy

Nanostructures

Pierce, Jr., Thomas A.

tpierce@nist.gov

Hardness measurements

Metallography 
Pitchure, David J.

david.pitchure@nist.gov

Mechanical testing

Computer-aided design

Mechanical engineering

Ricker, Richard E.

richard.ricker@nist.gov

Corrosion

Deformation mechanisms

Fracture mechanisms (SCC, CF HE)

TEM, SEM

\section{Ridder, Stephen D.}

stephen.ridder@nist.gov

Spray dynamics and deposition

Thermal spray processes

Process modeling and control

\section{Shapiro, Alexander J.}

alexander.shapiro@nist.gov

MOIF method microscopy

Mossbauer effect

Scanning electron microscopy (SEM) and $\mathrm{x}$-ray microanalysis

Image analysis

Shepherd, Dominique A.

dominique.shepherd@nist.gov

Titanium allloys

Mechanical testing

Mechanical properties

SEM, TEM

\section{Shull, Robert D.}

robert.shull@nist.gov

Nanocomposites

Magnetic susceptibility

Mossbauer effect

X-ray and neutron diffraction

Magneto-caloric and magneto-optical effects

Magnetic domain imaging
Stafford, Gery R.

gery.stafford@nist.gov

Electrochemical transients

Electrodeposition

Molten salt electrochemistry

\section{Stoudt, Mark R.}

mark.stoudt@nist.gov

Deformation-induced surface roughness

Mechanical properties

Metal fatigue

Thin films

Environmentally assisted cracking

Twisselmann, Douglas J.

douglas.twisselmann@nist.gov

Ferromagnetic resonance

Magnetometry

Warren, James A.

james.warren@nist.gov

Computer simulations of solidification

Dendrite pattern formation

Modeling of solder/substrate wetting processes

Modeling grain boundaries

\section{Williams, Maureen E.}

maureen.williams@nist.gov

Differential thermal analysis

Powder x-ray diffraction

Solder Wettability

SEM 


\section{Organizational Charts}

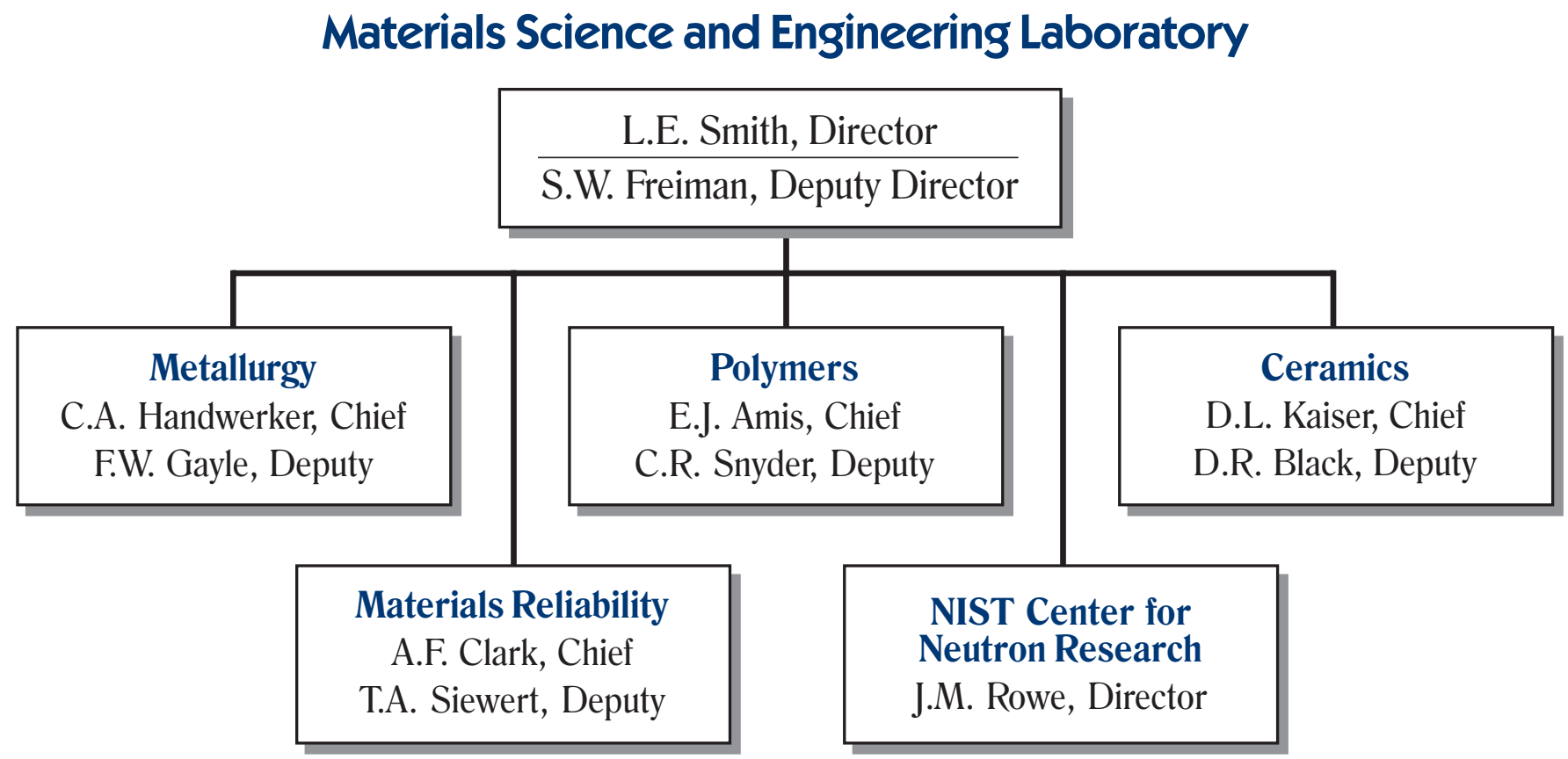

\section{National Institute of Standards and Technology}

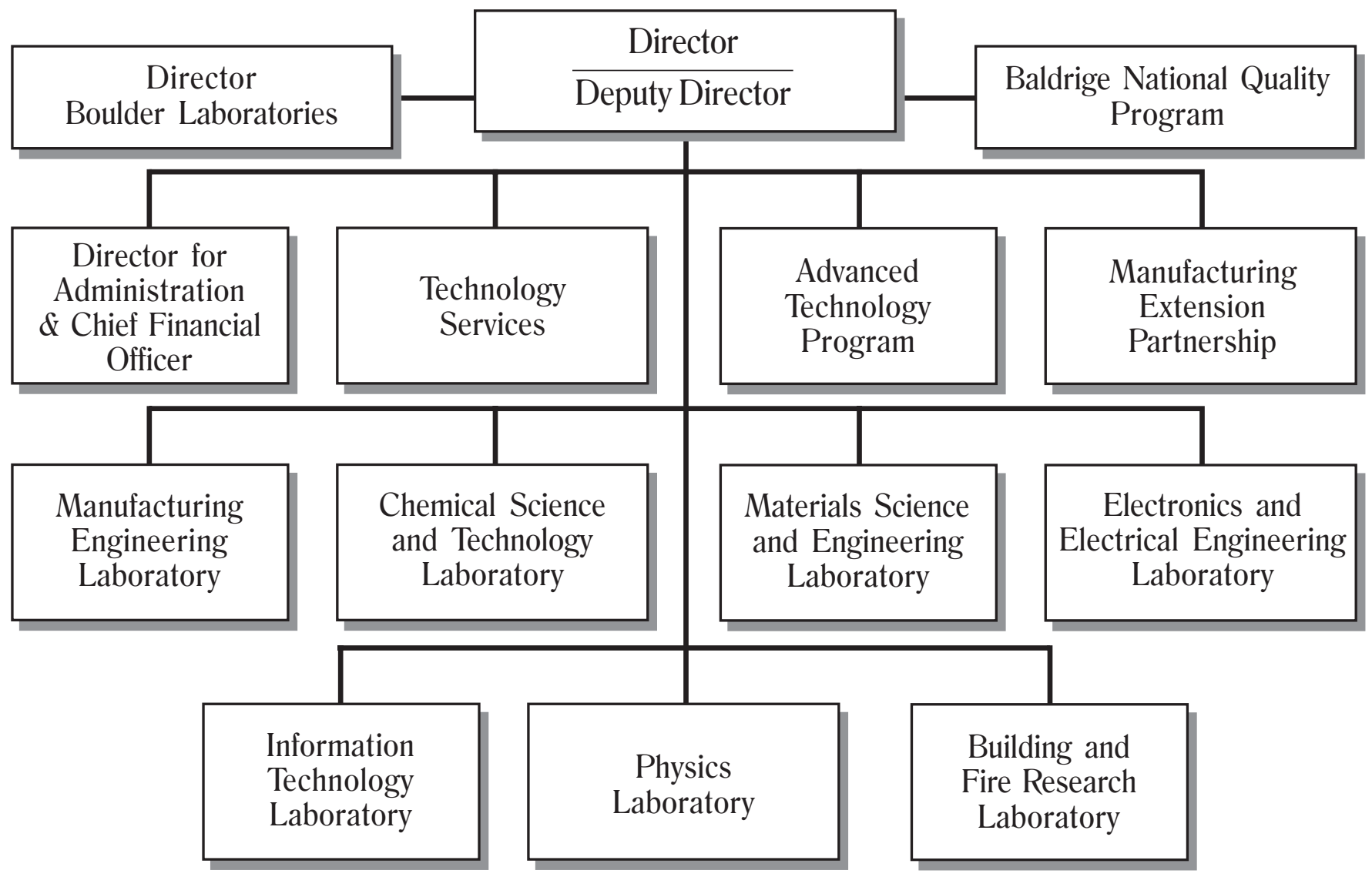



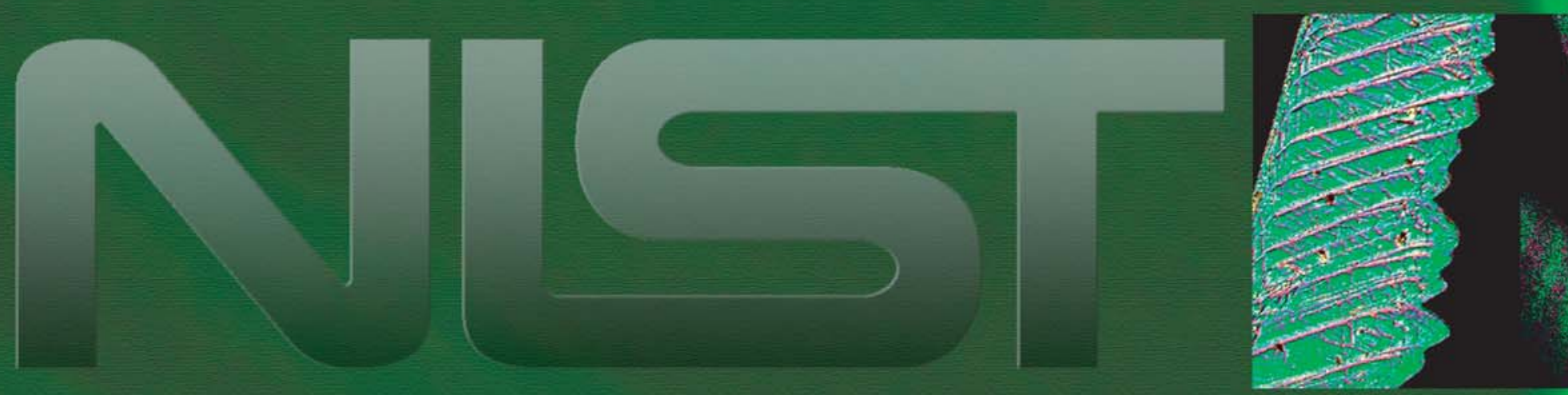